Supporting Information for:

\title{
Computational insights into the acceptor chemistry of phosphenium cations
}

\author{
Bobby D. Ellis ${ }^{a}$, Paul. J. Ragogna ${ }^{b}$ and Charles L.B. Macdonald ${ }^{* a}$ \\ ${ }^{a}$ Department of Chemistry and Biochemistry, University of Windsor, Windsor, ON, N9B \\ 3P4, Canada \\ ${ }^{b}$ Department of Chemistry, Dalhousie University, Halifax, NS, B3H 4J6, Canada \\ *cmacd@uwindsor.ca
}




\section{Experimental Procedures}

\section{General Procedures}

All manipulations were carried out in a $\mathrm{N}_{2}$ filled Innovative Technologies Dry box. All solvents were distilled prior to use and distilled in vacuo. $\mathrm{CH}_{2} \mathrm{Cl}_{2}$ was dried at reflux over $\mathrm{CaH}_{2}, \mathrm{P}_{2} \mathrm{O}_{5}$ and again over $\mathrm{CaH}_{2}$. Pentane was dried at reflux over $\mathrm{Na} /$ benzophenone and 5\% tetraethyleneglycoldimethylether. Imidizole-2-ylidene (NHC) and $\left[\mathrm{Ph}_{3} \mathrm{P}-\mathrm{PPh}_{2}\right]\left[\mathrm{GaCl}_{4}\right]$ were prepared using literature methods. IR spectra were collected on a Bruker VECTOR 22 FT-IR using nujol mulls and are reported with ranked intensities in parentheses. Solution NMR data were collected on a Bruker AC-250 NMR spectrometer at room temperature unless otherwise indicated. ${ }^{31} \mathrm{P}$ NMR chemical shifts are externally referenced to $85 \% \mathrm{H}_{3} \mathrm{PO}_{4}$. Crystals of $\left[\mathrm{NHC}-\mathrm{PPh}_{2}\right]\left[\mathrm{GaCl}_{4}\right], 6\left[\mathrm{GaCl}_{4}\right]$ were mounted inside glass capillaries $(<1.0 \mathrm{~mm}$ o.d.) and flame sealed. X-ray diffraction data were collected on a Rigaku AFC5R diffractometer equipped with a rotating anode source using graphite-monochromated $\mathrm{Cu}-\mathrm{K}_{\alpha}(\lambda=1.5478 \AA)$ radiation.

\section{Specific Procedures}

6[ $\left.\mathrm{GaCl}_{4}\right]:(0.16 \mathrm{~g}, 0.76 \mathrm{mmol})$ of 2,3-Dihydro-1,3-diisopropyl-4,5-dimethylimidazol-2ylidene in $15 \mathrm{~mL} \mathrm{C}_{6} \mathrm{H}_{6}$ was added dropwise to a slurry of $\left[\mathrm{Ph}_{3} \mathrm{P}-\mathrm{PPh}_{2}\right]\left[\mathrm{GaCl}_{4}\right](0.51 \mathrm{~g}$, $0.76 \mathrm{mmol})$ to give a clear, colorless solution. Concentration of the reaction mixture, and subsequent addition of pentane $(20 \mathrm{~mL})$ yielded a white powder $0.35 \mathrm{~g}, 70 \%$; m.p. 128 $130^{\circ} \mathrm{C}$. X-ray quality crystals were grown using slow solvent evaporation from $\mathrm{CH}_{2} \mathrm{Cl}_{2}$. Anal. Calcd. (Found): C, 53.45 (52.07), H, 5.50 (5.88); IR ( $\left.\mathrm{cm}^{-1}\right)$ : 377(2), 469(17), 486(12), 503(11), 675(4), 369(3), 742(1), 792(18), 903(15), 916(16), 969(14), 998(13), 1026(8), 1086(7), 1113(9), 1216(10), 1261(6), 1306(5), 1583(20), 1611(19); Summary of 
Crystal Data: $\mathrm{C}_{26} \mathrm{H}_{33} \mathrm{~N}_{2} \mathrm{PCl}_{4} \mathrm{Ga} \cdot \mathrm{C}_{6} \mathrm{H}_{6} ; \mathrm{M}=616.07 \mathrm{~g} \mathrm{~mol}^{-1}$, monoclinic, $P 2{ }_{1} / a$, a=16.80(2) $\AA$, $b=20.748(6) \AA, \quad c=19.343(8) \AA, \quad \beta=114.76(4)^{\circ}, \quad V=6122(7) \AA^{3}, \quad T=293(2) K, \quad Z=8, \mu(C u-$ $\mathrm{K} \alpha)=50.62 \mathrm{~cm}^{-1}, 6192$ measured reflections, 5969 unique, 584 refined parameters, $\mathrm{R}[\mathrm{I}>3 \sigma(\mathrm{I})]=0.075, w R 2\left(\mathrm{~F}^{2}\right)=0.209$. Complete crystallographic data is available in the accompanying cif file and the contents of the asymmetric unit are pictured below.
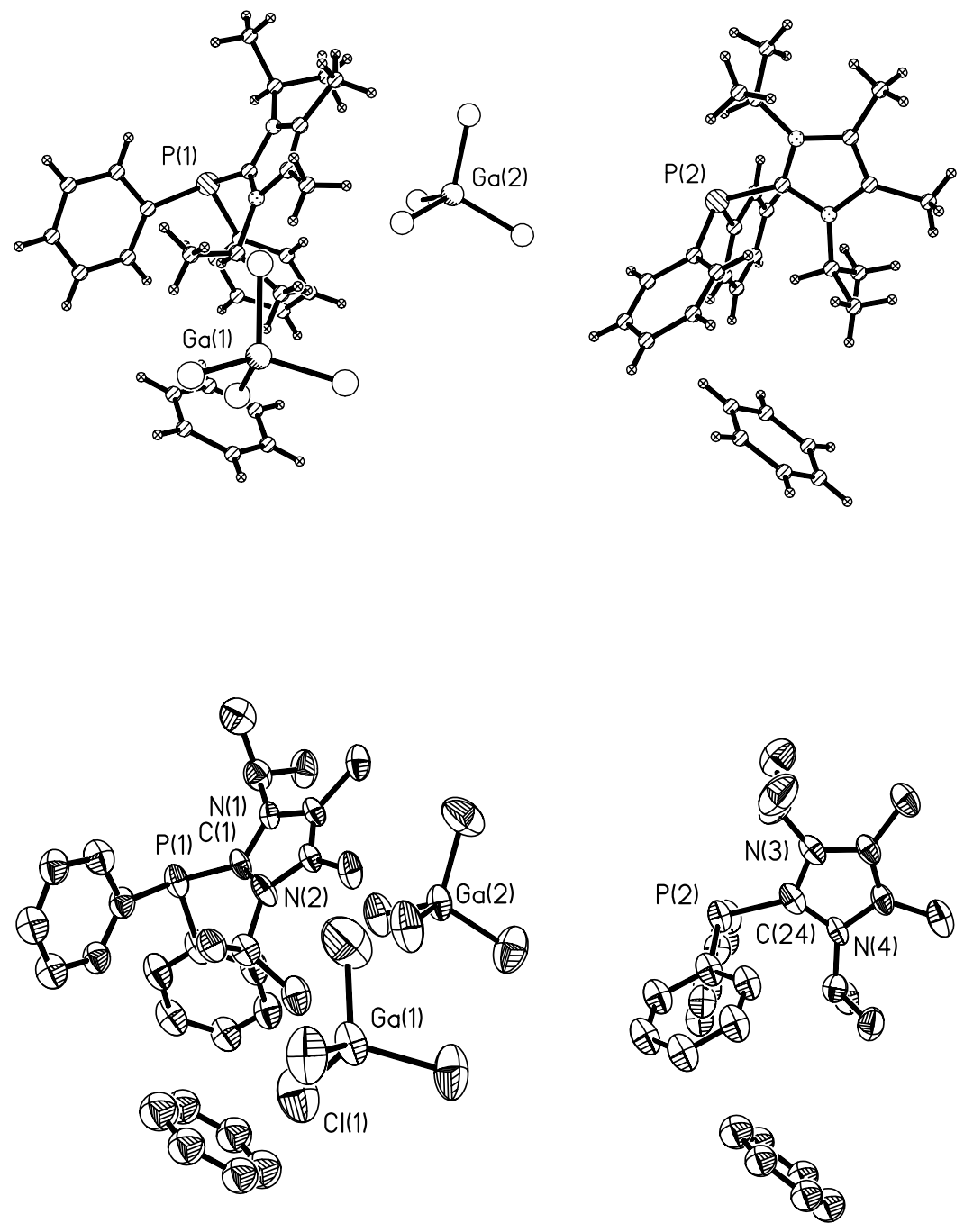


\section{Optimized geometries and Energies for all model compounds}

\begin{tabular}{ccccccc}
\hline Cation & Symmetry & $\begin{array}{c}\text { Energy } \\
\text { (au) }\end{array}$ & $\begin{array}{c}\text { ZPVE } \\
\text { (au) }\end{array}$ & $\begin{array}{c}\text { Corrected } \\
\text { Energy } \\
(\mathbf{a u})\end{array}$ & $\begin{array}{c}\text { HOMO } \\
(\mathbf{e V})\end{array}$ & $\begin{array}{c}\text { LUMO } \\
(\mathbf{e V})\end{array}$ \\
\hline $\mathbf{1}$ & $C_{2}$ & -804.31208 & 0.18388 & -804.13235 & -11.370 & -8.257 \\
$\mathbf{2}$ & $C_{2}$ & -610.24620 & 0.16956 & -610.08047 & -11.719 & -6.910 \\
\hline
\end{tabular}

Geometry Optimization of $\mathrm{P}\left(\mathrm{Ph}_{2}\right)_{2}{ }^{+}, \mathbf{1}$, in $C_{2}$ symmetry

$1 \backslash 1 \backslash G I N C-N E I L \backslash F O p t \backslash R B 3 P W 91 \backslash 6-31 G(d) \backslash C 12 H 10 P 1(1+) \backslash B O B B Y \backslash 13-J a n-2003 \backslash 0 \backslash \backslash$ \# B3PW91/6-31G(D) OPT FREQ=NORAMAN POP $=(\mathrm{FULL}, \mathrm{NBO}) \backslash \backslash \mathrm{Geom}$ and Freq of $\mathrm{P}($ Ph2) $2+-C 2 \backslash \backslash 1,1 \backslash P, 0 ., 0 ., 1.4446928675 \backslash C, 1.4252393226,-0.1592873458,0.423$ $7364946 \backslash \mathrm{C},-1.4252393226,0.1592873458,0.4237364946 \backslash \mathrm{C}, 2.5465361454,-0.72$ $36508297,1.0966498163 \backslash C,-2.5465361454,0.7236508297,1.0966498163 \backslash C, 1.58$ $15606208,0.2939089473,-0.9146455658 \backslash C,-1.5815606208,-0.2939089473,-0.9$ $146455658 \backslash \mathrm{C}, 3.7466988374,-0.9036061025,0.429317156 \backslash \mathrm{C},-3.7466988374,0.9$ $036061025,0.429317156 \backslash C, 2.7954148863,0.1393268178,-1.5580798288 \backslash C,-2.7$ $954148863,-0.1393268178,-1.5580798288 \backslash \mathrm{C}, 3.8697067782,-0.4732790713,-0$. $8954007117 \backslash \mathrm{C},-3.8697067782,0.4732790713,-0.8954007117 \backslash \mathrm{H}, 2.452927254,-1$ $.0313021208,2.1357957013 \backslash \mathrm{H},-2.452927254,1.0313021208,2.1357957013 \backslash \mathrm{H}, \mathrm{O}$. $7682872771,0.8094795921,-1.4143183493 \backslash \mathrm{H},-0.7682872771,-0.8094795921,-1$ $.4143183493 \backslash \mathrm{H}, 4.59167985,-1.3573652421,0.9375403916 \backslash \mathrm{H},-4.59167985,1.35$ $73652421,0.9375403916 \backslash \mathrm{H}, 2.9237720338,0.5058375122,-2.5720400465 \backslash \mathrm{H},-2.9$ $237720338,-0.5058375122,-2.5720400465 \backslash \mathrm{H}, 4.8185988814,-0.5906981931,-1$. $4116383675 \backslash \mathrm{H},-4.8185988814,0.5906981931,-1.4116383675 \backslash \backslash$ Version $=x 86-\mathrm{Lin}$ ux-G98RevA. $11.1 \backslash$ State $=1-\mathrm{A} \backslash \mathrm{HF}=-804.14999 \backslash \mathrm{RMSD}=2.883 \mathrm{e}-09 \backslash \mathrm{RMSF}=1.068 \mathrm{e}-05 \backslash$ Dipole $=0 ., 0 .,-0.3667379 \backslash \mathrm{PG}=\mathrm{C} 02 \quad[\mathrm{C} 2(\mathrm{P} 1), \mathrm{X}(\mathrm{C} 12 \mathrm{H} 10)] \backslash \backslash \mathrm{C}$

\section{Single Point Energy Calculation of $\mathrm{P}\left(\mathrm{Ph}_{2}\right)_{2}{ }^{+}, \mathbf{1}$, in $C_{2}$ symmetry}

$1 \backslash 1 \backslash \mathrm{GINC}-\mathrm{NEIL} \backslash \mathrm{SP} \backslash \mathrm{RB} 3 \mathrm{PW} 91 \backslash 6-311+\mathrm{G}(3 \mathrm{df}, 2 \mathrm{p}) \backslash \mathrm{C} 12 \mathrm{H} 10 \mathrm{P} 1(1+) \backslash \mathrm{BOBBY} \backslash 15-\mathrm{Jan}-200$ $3 \backslash 0 \backslash \backslash \#$ B3PW91/6-311+G (3DF,2P) GEOM=CHECK GUESS=READ SCF=TIGHT \#P GFINP UT IOP (6/7=3) T \\Single Point and Molden of $P(P h 2) 2+-C 2 \backslash \backslash 1,1 \backslash P, 0 ., 0 ., 1$ $.4446928675 \backslash \mathrm{C}, 1.4252393226,-0.1592873458,0.4237364946 \backslash \mathrm{C},-1.4252393226$, $0.1592873458,0.4237364946 \backslash \mathrm{C}, 2.5465361454,-0.7236508297,1.0966498163 \backslash \mathrm{C}$, $-2.5465361454,0.7236508297,1.0966498163 \backslash \mathrm{C}, 1.5815606208,0.2939089473,-0$ $.9146455658 \backslash \mathrm{C},-1.5815606208,-0.2939089473,-0.9146455658 \backslash \mathrm{C}, 3.7466988374$ $,-0.9036061025,0.429317156 \backslash \mathrm{C},-3.7466988374,0.9036061025,0.429317156 \backslash \mathrm{C}$, $2.7954148863,0.1393268178,-1.5580798288 \backslash \mathrm{C},-2.7954148863,-0.1393268178$, $-1.5580798288 \backslash \mathrm{C}, 3.8697067782,-0.4732790713,-0.8954007117 \backslash \mathrm{C},-3.86970677$ $82,0.4732790713,-0.8954007117 \backslash \mathrm{H}, 2.452927254,-1.0313021208,2.1357957013$ $\backslash \mathrm{H},-2.452927254,1.0313021208,2.1357957013 \backslash \mathrm{H}, 0.7682872771,0.8094795921$, $-1.4143183493 \backslash \mathrm{H},-0.7682872771,-0.8094795921,-1.4143183493 \backslash \mathrm{H}, 4.59167985$ $,-1.3573652421,0.9375403916 \backslash \mathrm{H},-4.59167985,1.3573652421,0.9375403916 \backslash \mathrm{H}$, $2.9237720338,0.5058375122,-2.5720400465 \backslash \mathrm{H},-2.9237720338,-0.5058375122$, $-2.5720400465 \backslash \mathrm{H}, 4.8185988814,-0.5906981931,-1.4116383675 \backslash \mathrm{H},-4.81859888$ $14,0.5906981931,-1.4116383675 \backslash \backslash$ Version=x86-Linux-G98RevA.11.1\State=1$\mathrm{A} \backslash \mathrm{HF}=-804.3120809 \backslash \mathrm{RMSD}=8.474 \mathrm{e}-09 \backslash \mathrm{Dipole}=0,0,,-0.3325899 \backslash \mathrm{PG}=\mathrm{C} 02 \quad[\mathrm{C} 2(\mathrm{P} 1$ )$, \mathrm{X}(\mathrm{C} 12 \mathrm{H} 10)] \backslash \backslash \mathrm{Q}$

MP2 Single Point Energy Calculation of $\mathrm{P}\left(\mathrm{Ph}_{2}\right)_{2}{ }^{+}, \mathbf{1}$, in $C_{2}$ symmetry 
$1 \backslash 1 \backslash G I N C-N E I L \backslash S P \backslash R M P 2-F C \backslash 6-31+G(d) \backslash C 12 H 10 P 1(1+) \backslash C H U C K \backslash 02-A u g-2004 \backslash 0 \backslash \backslash \#$ $\mathrm{MP} 2 / 6-31+\mathrm{G}(\mathrm{D}) \quad \mathrm{POP}=(\mathrm{NBOREAD}) \quad \mathrm{MAXDISK}=800 \mathrm{MB}$ TEST $\backslash$ Hardness of $\mathrm{PPh} 2+-\mathrm{C} 2 \backslash$ $\backslash 1,1 \backslash P, 0,0.0 ., 1.444693 \backslash \mathrm{C}, 0,0.1 .434113,0.423736 \backslash \mathrm{C}, 0,0 .,-1.434113,0.42$ $3736 \backslash C, 0,0.436329,2.611156,1.09665 \backslash C, 0,-0.436329,-2.611156,1.09665 \backslash C, 0$ $,-0.467755,1.53913,-0.914646 \backslash \mathrm{C}, 0,0.467755,-1.53913,-0.914646 \backslash \mathrm{C}, 0,0.481$ $868,3.82388,0.429317 \backslash \mathrm{C}, 0,-0.481868,-3.82388,0.429317 \backslash \mathrm{C}, 0,-0.448952,2.7$ $62643,-1.55808 \backslash \mathrm{C}, 0,0.448952,-2.762643,-1.55808 \backslash \mathrm{C}, 0,0.040541,3.89833,-0$ $.895401 \backslash \mathrm{C}, 0,-0.040541,-3.89833,-0.895401 \backslash \mathrm{H}, 0,0.752474,2.552297,2.13579$ $6 \backslash \mathrm{H}, 0,-0.752474,-2.552297,2.135796 \backslash \mathrm{H}, 0,-0.889805,0.673624,-1.414318 \backslash \mathrm{H}$, $0,0.889805,-0.673624,-1.414318 \backslash \mathrm{H}, 0,0.838967,4.714032,0.93754 \backslash \mathrm{H}, 0,-0.83$ $8967,-4.714032,0.93754 \backslash \mathrm{H}, 0,-0.827452,2.849498,-2.57204 \backslash \mathrm{H}, 0,0.827452,-2$ $.849498,-2.57204 \backslash \mathrm{H}, 0,0.05184,4.854393,-1.411638 \backslash \mathrm{H}, 0,-0.05184,-4.854393$ ,$-1.411638 \backslash \backslash$ Version=x86-Linux-G98RevA.11.1 \State=1-A \HF=-800.7249045 $\mathrm{P} 2=-802.3460232 \backslash \mathrm{RMSD}=4.590 e-09 \backslash \mathrm{PG}=\mathrm{C} 02 \quad[\mathrm{C} 2(\mathrm{P} 1), \mathrm{X}(\mathrm{C} 12 \mathrm{H} 10)] \backslash \backslash @$

\section{Geometry Optimization of $\mathrm{P}\left(\mathrm{NMe}_{2}\right)_{2}{ }^{+}, \mathbf{2}$, in $C_{2}$ symmetry}

$1 \backslash 1 \backslash G I N C-N E I L \backslash F O p t \backslash R B 3 P W 91 \backslash 6-31 G(d) \backslash C 4 H 12 N 2 P 1(1+) \backslash B O B B Y \backslash 14-J a n-2003 \backslash 0 \backslash$ \\# B3PW91/6-31G(D) OPT FREQ=NORAMAN POP=(FULL, NBO) TEST \Geom and Freq of $\mathrm{P}(\mathrm{NMe} 2) 2+-\mathrm{C} 2 \mathrm{~A} \backslash \backslash 1,1 \backslash \mathrm{P}, 0 ., 0 ., 0.9255320848 \backslash \mathrm{N}, 1.3247005083,-0.24874830$ $43,-0.0091892027 \backslash \mathrm{N},-1.3247005083,0.2487483043,-0.0091892027 \backslash \mathrm{C}, 2.609546$ $3496,-0.1479567082,0.7106262458 \backslash \mathrm{C},-2.6095463496,0.1479567082,0.7106262$ $458 \backslash \mathrm{C}, 1.4604120411,-0.633329567,-1.416853613 \backslash \mathrm{C},-1.4604120411,0.6333295$ $67,-1.416853613 \backslash \mathrm{H}, 2.4443406386,0.0759583021,1.7686163669 \backslash \mathrm{H},-2.44434063$ $86,-0.0759583021,1.7686163669 \backslash \mathrm{H}, 0.5199021632,-1.0159574661,-1.80981031$ $37 \backslash \mathrm{H},-0.5199021632,1.0159574661,-1.8098103137 \backslash \mathrm{H}, 3.1503583851,-1.094889$ $9921,0.6250823715 \backslash \mathrm{H},-3.1503583851,1.0948899921,0.6250823715 \backslash \mathrm{H}, 3.209653$ $8978,0.6575698722,0.2756948132 \backslash \mathrm{H},-3.2096538978,-0.6575698722,0.2756948$ $132 \backslash \mathrm{H}, 1.8062899936,0.2117208609,-2.0217858237 \backslash \mathrm{H},-1.8062899936,-0.21172$ $08609,-2.0217858237 \backslash \mathrm{H}, 2.2071663842,-1.4310356254,-1.4775994283 \backslash \mathrm{H},-2.20$ $71663842,1.4310356254,-1.4775994283 \backslash \backslash$ Version=x86-Linux-G98RevA.11.1\St $\mathrm{ate}=1-\mathrm{A} \backslash \mathrm{HF}=-610.1198928 \backslash \mathrm{RMSD}=9.280 e-09 \backslash \mathrm{RMSF}=1.207 e-04 \backslash \mathrm{Dipole}=0 ., 0 .,-0$. $3613169 \backslash \mathrm{PG}=\mathrm{C} 02 \quad[\mathrm{C} 2(\mathrm{P} 1), \mathrm{X}(\mathrm{C} 4 \mathrm{H} 12 \mathrm{~N} 2)] \backslash \backslash \mathrm{C}$

\section{Single Point Energy Calculation of $\mathrm{P}\left(\mathrm{NMe}_{2}\right)_{2}{ }^{+}, \mathbf{2}$, in $C_{2}$ symmetry}

$1 \backslash 1 \backslash G I N C-N E I L \backslash S P \backslash R B 3 P W 91 \backslash 6-311+G(3 d f, 2 p) \backslash C 4 H 12 N 2 P 1(1+) \backslash B O B B Y \backslash 14-J a n-20$ $03 \backslash 0 \backslash \backslash \#$ B3PW91/6-311+G(3DF, 2P) GEOM=CHECK GUESS=READ SCF=TIGHT \#P GFIN PUT IOP $(6 / 7=3)$ T \\Single Point and Molden of $P(N M e 2) 2+-C 2 A \backslash \backslash 1,1 \backslash P, 0 ., 0$ $.0 .9255320848 \backslash \mathrm{N}, 1.3247005083,-0.2487483043,-0.0091892027 \backslash \mathrm{N},-1.3247005$ $083,0.2487483043,-0.0091892027 \backslash \mathrm{C}, 2.6095463496,-0.1479567082,0.71062624$ $58 \backslash \mathrm{C},-2.6095463496,0.1479567082,0.7106262458 \backslash \mathrm{C}, 1.4604120411,-0.6333295$ $67,-1.416853613 \backslash \mathrm{C},-1.4604120411,0.633329567,-1.416853613 \backslash \mathrm{H}, 2.444340638$ $6,0.0759583021,1.7686163669 \backslash \mathrm{H},-2.4443406386,-0.0759583021,1.7686163669$ $\backslash \mathrm{H}, 0.5199021632,-1.0159574661,-1.8098103137 \backslash \mathrm{H},-0.5199021632,1.01595746$ $61,-1.8098103137 \backslash \mathrm{H}, 3.1503583851,-1.0948899921,0.6250823715 \backslash \mathrm{H},-3.150358$ $3851,1.0948899921,0.6250823715 \backslash \mathrm{H}, 3.2096538978,0.6575698722,0.275694813$ $2 \backslash \mathrm{H},-3.2096538978,-0.6575698722,0.2756948132 \backslash \mathrm{H}, 1.8062899936,0.21172086$ $09,-2.0217858237 \backslash \mathrm{H},-1.8062899936,-0.2117208609,-2.0217858237 \backslash \mathrm{H}, 2.20716$ $63842,-1.4310356254,-1.4775994283 \backslash \mathrm{H},-2.2071663842,1.4310356254,-1.4775$ 994283 \Version=x86-Linux-G98RevA.11.1\State=1-A \HF=-610.2461985 RMSD= $7.144 e-09 \backslash \mathrm{Dipole}=0 ., 0 .,-0.3336933 \backslash \mathrm{PG}=\mathrm{C} 02 \quad[\mathrm{C} 2(\mathrm{P} 1), \mathrm{X}(\mathrm{C} 4 \mathrm{H} 12 \mathrm{~N} 2)] \backslash \backslash @$ 


\begin{abstract}
MP2 Single Point Energy Calculation of $\mathrm{P}\left(\mathrm{NMe}_{2}\right)_{2}{ }^{+}, \mathbf{2}$, in $C_{2}$ symmetry
$1 \backslash 1 \backslash G I N C-N E I L \backslash S P \backslash R M P 2-F C \backslash 6-31+G(d) \backslash C 4 H 12 N 2 P 1(1+) \backslash C H U C K \backslash 02-A u g-2004 \backslash 0 \backslash \backslash$ \# MP2/6-31+G(D) $\mathrm{POP}=(\mathrm{NBOREAD}) \quad \mathrm{TEST} \backslash \backslash$ Hardness of $\mathrm{P}(\mathrm{NMe} 2) 2+-\mathrm{C} 2 \mathrm{~A} \backslash \backslash 1,1 \backslash \mathrm{P}, 0$ $, 0.0 ., 0.925532 \backslash \mathrm{N}, 0,0 ., 1.347853,-0.009189 \backslash \mathrm{N}, 0,0 .,-1.347853,-0.009189 \backslash \mathrm{C}$ $, 0,-0.336181,2.592027,0.710626 \backslash \mathrm{C}, 0,0.336181,-2.592027,0.710626 \backslash \mathrm{C}, 0,0.3$ $52929,1.552208,-1.416854 \backslash \mathrm{C}, 0,-0.352929,-1.552208,-1.416854 \backslash \mathrm{H}, 0,-0.5257$ $6,2.388336,1.768616 \backslash \mathrm{H}, 0,0.52576,-2.388336,1.768616 \backslash \mathrm{H}, 0,0.902557,0.6984$ $68,-1.80981 \backslash \mathrm{H}, 0,-0.902557,-0.698468,-1.80981 \backslash \mathrm{H}, 0,0.494679,3.298308,0.6$ $25082 \backslash \mathrm{H}, 0,-0.494679,-3.298308,0.625082 \backslash \mathrm{H}, 0,-1.238621,3.033166,0.275695$ $\backslash \mathrm{H}, 0,1.238621,-3.033166,0.275695 \backslash \mathrm{H}, 0,-0.541438,1.73619,-2.021786 \backslash \mathrm{H}, 0,0$ $.541438,-1.73619,-2.021786 \backslash \mathrm{H}, 0,0.999119,2.433353,-1.477599 \backslash \mathrm{H}, 0,-0.9991$ $19,-2.433353,-1.477599 \backslash \backslash$ Version=x86-Linux-G98RevA. $11.1 \backslash$ State $=1-\mathrm{A} \backslash \mathrm{HF}=-6$ $07.8495781 \backslash \mathrm{MP} 2=-608.8191316 \backslash \mathrm{RMSD}=2.918 e-09 \backslash \mathrm{PG}=\mathrm{C} 02 \quad[\mathrm{C} 2(\mathrm{P} 1), \mathrm{X}(\mathrm{C} 4 \mathrm{H} 12 \mathrm{~N} 2)] \backslash$ $\backslash$ Q
\end{abstract}

\begin{tabular}{|c|c|c|c|c|c|c|c|}
\hline Donor & Symm. & $\begin{array}{c}\text { Energy } \\
\text { (au) }\end{array}$ & $\begin{array}{c}\text { ZPVE } \\
\text { (au) }\end{array}$ & $\begin{array}{l}{ }^{(\text {a) }} \text { Corrected } \\
\text { Energy (au) }\end{array}$ & $\begin{array}{c}\text { HOMO } \\
(\mathrm{eV})\end{array}$ & $\begin{array}{c}{ }^{(b)} \Delta \mathbf{H}_{\text {prep }} \\
\text { Adduct } \\
\text { with 1 } \\
\left(\mathrm{kJ} \mathrm{mol}^{-1}\right)\end{array}$ & $\begin{array}{c}{ }^{(\mathrm{b})} \Delta \mathbf{H}_{\text {prep }} \\
\text { Adduct } \\
\text { with } 2 \\
\left(\mathrm{~kJ} \mathrm{~mol}^{-1}\right)\end{array}$ \\
\hline $\mathrm{PMe}_{3}$ & $C_{3}$ & -461.08350 & 0.11371 & -460.97236 & -6.208 & 27.7 & 23.1 \\
\hline DHAP & $C_{s}$ & -303.63137 & 0.10621 & -303.52756 & -6.536 & 10.3 & 9.5 \\
\hline Carbene & $C_{2 v}$ & -226.16790 & 0.07183 & -226.09770 & -6.116 & 8.3 & 8.9 \\
\hline
\end{tabular}

(a) Corrected energy: $\mathrm{E}_{\text {corrected }}=\mathrm{E}_{\text {calculated }}+$ ZPVE. (b) The preparation energy for the donor is calculated for the process: Donor $\rightarrow$ Donor* $; \Delta \mathrm{H}_{\text {prep }}=\mathrm{E}_{\mathrm{donor} *}-\mathrm{E}_{\mathrm{donor}}$, where the "*" indicates that the donor/acceptor fragment is in the exact same geometry observed in the coordination complex.

\title{
Geometry Optimization of $\mathrm{PMe}_{3}$ ligand, in $C_{3}$ symmetry
}

$1 \backslash 1 \backslash G I N C-N E I L \backslash F O p t \backslash R B 3 P W 91 \backslash 6-31 G(d) \backslash C 3 H 9 P 1 \backslash B O B B Y \backslash 09-J a n-2003 \backslash 1$ 91/6-31G(D) FOPT=Z-MATRIX FREQ=NORAMAN POP=(FULL, NBO) TEST \Ge req of $\mathrm{PMe} 3 \backslash \backslash 0,1 \backslash \mathrm{P} \backslash \mathrm{X}, 1,1 . \backslash \mathrm{C}, 1, \mathrm{cp}, 2, \mathrm{cpx} \backslash \mathrm{C}, 1, \mathrm{cp}, 2, \mathrm{cpx}, 3,120 ., 0 \backslash \mathrm{C}$

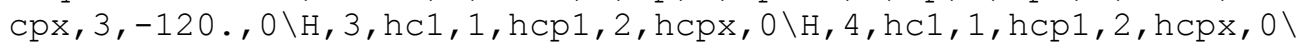
1, hсp1, 2, hсpx, $0 \backslash \mathrm{H}, 3, \mathrm{hc2}, 1, \mathrm{hcp} 2,6, \mathrm{hcph} 1,0 \backslash \mathrm{H}, 4, \mathrm{hc2}, 1, \mathrm{hcp} 2,7$, hcph

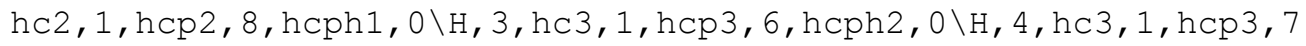
$\backslash \mathrm{H}, 5, \mathrm{hc} 3,1, \mathrm{hcp} 3,8, \mathrm{hcph} 2,0 \backslash \backslash \mathrm{cp}=1.85909093 \backslash \mathrm{cpx}=118.28099422 \backslash \mathrm{hc} 1=$ $62 \backslash \mathrm{hcp} 1=109.53572721 \backslash \mathrm{hcpx}=59.57841987 \backslash \mathrm{hc} 2=1.09728284 \backslash \mathrm{hcp} 2=112$. $\backslash \mathrm{hcph} 1=121.20325361 \backslash \mathrm{hc} 3=1.09547322 \backslash \mathrm{hcp} 3=109.51751433 \backslash \mathrm{hcph} 2=-11$ $23 \backslash \backslash$ Version=x86-Linux-G98RevA.11.1 \HF=-461.0086127\RMSD=5.173e $=5.812 e-05 \backslash \mathrm{Dipole}=0 ., 0 .,-0.5571924 \backslash \mathrm{PG}=\mathrm{C} 03 \quad[\mathrm{C} 3(\mathrm{P} 1), \mathrm{X}(\mathrm{C} 3 \mathrm{H} 9)] \backslash \backslash \mathrm{a}$

\section{Single Point Energy of $\mathrm{PMe}_{3}$ ligand, in $C_{3}$ symmetry}

$1 \backslash 1 \backslash G I N C-N E I L \backslash S P \backslash R B 3 P W 91 \backslash 6-311+G(3 d f, 2 p) \backslash C 3 H 9 P 1 \backslash B O B B Y \backslash 30-J a n-2003 \backslash 0 \backslash \backslash \#$ B3PW91/6-311+G (3DF, 2P) GEOM=CHECK GUESS=READ SCF=TIGHT \#P GFINPUT IOP

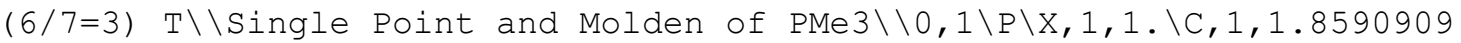
$327,2,118.28099422 \backslash \mathrm{C}, 1,1.8590909327,2,118.28099422,3,120 ., 0 \backslash \mathrm{C}, 1,1.8590$ $909327,2,118.28099422,3,-120 ., 0 \backslash \mathrm{H}, 3,1.0954256211,1,109.53572721,2,59.5$ $7841987,0 \backslash \mathrm{H}, 4,1.0954256211,1,109.53572721,2,59.57841987,0 \backslash \mathrm{H}, 5,1.095425$ 
$6211,1,109.53572721,2,59.57841987,0 \backslash \mathrm{H}, 3,1.0972828375,1,112.84853057,6$, $121.20325361,0 \backslash \mathrm{H}, 4,1.0972828375,1,112.84853057,7,121.20325361,0 \backslash \mathrm{H}, 5,1$. $0972828375,1,112.84853057,8,121.20325361,0 \backslash \mathrm{H}, 3,1.0954732174,1,109.5175$ $1433,6,-117.65773923,0 \backslash \mathrm{H}, 4,1.0954732174,1,109.51751433,7,-117.65773923$ , $0 \backslash \mathrm{H}, 5,1.0954732174,1,109.51751433,8,-117.65773923,0 \backslash \backslash$ Version=x86-Linu

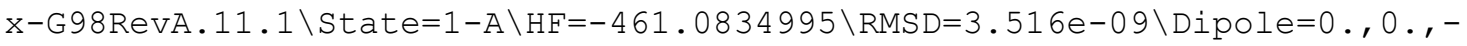
$0.505494 \backslash \mathrm{PG}=\mathrm{C} 03 \quad[\mathrm{C} 3(\mathrm{P} 1), \mathrm{X}(\mathrm{C} 3 \mathrm{H} 9)] \backslash \backslash @$

\section{MP2 Single Point Energy of $\mathrm{PMe}_{3}$ ligand, in $C_{3}$ symmetry}

$1 \backslash 1 \backslash G I N C-N E I L \backslash S P \backslash R M P 2-F C \backslash 6-31+G(d) \backslash C 3 H 9 P 1 \backslash C H U C K \backslash 02-A u g-2004 \backslash 0 \backslash \backslash \#$ MP2/6 $-31+\mathrm{G}(\mathrm{D}) \quad \mathrm{POP}=(\mathrm{NBOREAD}) \quad \mathrm{TEST} \backslash \backslash$ Hardness of $\mathrm{PMe} \backslash \backslash 0,1 \backslash \mathrm{P}, 0,0 ., 0 ., 0.601827 \backslash$ $\mathrm{C}, 0,-0.612335,1.518356,-0.279003 \backslash \mathrm{C}, 0,1.621102,-0.22888,-0.279003 \backslash \mathrm{C}, 0,-$ $1.008767,-1.289475,-0.279003 \backslash \mathrm{H}, 0,0 ., 2.380186,0.007792 \backslash \mathrm{H}, 0,2.061302,-1$. $190093,0.007792 \backslash \mathrm{H}, 0,-2.061302,-1.190093,0.007792 \backslash \mathrm{H}, 0,-0.586291,1.41689$ $5,-1.371275 \backslash \mathrm{H}, 0,1.520212,-0.200704,-1.371275 \backslash \mathrm{H}, 0,-0.933921,-1.21619,-1$ $.371275 \backslash \mathrm{H}, 0,-1.64241,1.729375,0.028367 \backslash \mathrm{H}, 0,2.318888,0.557681,0.028367 \backslash$ $\mathrm{H}, 0,-0.676478,-2.287057,0.028367 \backslash \backslash$ Version=x86-Linux-G98RevA. $11.1 \backslash \mathrm{HF}=-4$ $59.5690359 \backslash \mathrm{MP} 2=-460.076634 \backslash \mathrm{RMSD}=1.646 \mathrm{e}-09 \backslash \mathrm{PG}=\mathrm{C} 01 \quad[\mathrm{X}(\mathrm{C} 3 \mathrm{H} 9 \mathrm{P} 1)] \backslash \backslash \mathrm{a}$

\section{Geometry Optimization of DHAP ligand, in $C_{s}$ symmetry}

$1 \backslash 1 \backslash G I N C-N E I L \backslash F O p t \backslash R B 3 P W 91 \backslash 6-31 G(d) \backslash C 5 H 6 N 2 \backslash B O B B Y \backslash 28-O c t-2003 \backslash 0 \backslash \backslash \#$ B3PW 91/6-31G(D) OPT $F R E Q=N O R A M A N$ POP=(FULL, NBO) TEST \\Geom and Freq of DHA

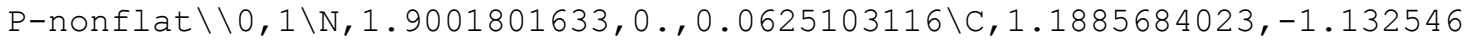
$1308,0.0411483997 \backslash \mathrm{C}, 1.1885684023,1.1325461308,0.0411483997 \backslash \mathrm{C},-0.197832$ $7664,-1.1961754089,-0.0030023658 \backslash \mathrm{C},-0.1978327664,1.1961754089,-0.00300$ $23658 \backslash \mathrm{C},-0.9329697689,0 .,-0.0265298623 \backslash \mathrm{N},-2.311610154,0 .,-0.0208641789$ $\backslash \mathrm{H},-2.7669548945,0.8438000606,-0.3387017251 \backslash \mathrm{H},-2.7669548945,-0.8438000$ $606,-0.3387017251 \backslash \mathrm{H}, 1.765234018,-2.0570205185,0.0583535149 \backslash \mathrm{H}, 1.7652340$ $18,2.0570205185,0.0583535149 \backslash \mathrm{H},-0.7037786647,-2.1587291867,-0.01469987$ $05 \backslash \mathrm{H},-0.7037786647,2.1587291867,-0.0146998705 \backslash \backslash$ Version=x86-Linux-G98Re $\mathrm{VA} .11 .1 \backslash \mathrm{State}=1-\mathrm{A}^{\prime} \backslash \mathrm{HF}=-303.5275165 \backslash \mathrm{RMSD}=6.976 \mathrm{e}-09 \backslash \mathrm{RMSF}=8.332 \mathrm{e}-05 \backslash \mathrm{Dipol}$ $e=-1.5344839,0 .,-0.4384138 \backslash P G=C S \quad[S G(C 1 N 2), X(C 4 H 6)] \backslash \backslash @$

\section{Single Point Energy of DHAP ligand, in $C_{s}$ symmetry}

$1 \backslash 1 \backslash G I N C-N E I L \backslash S P \backslash R B 3 P W 91 \backslash 6-311+G(3 \mathrm{df}, 2 \mathrm{p}) \backslash \mathrm{C} 5 \mathrm{H} 6 \mathrm{~N} 2 \backslash \mathrm{BOBBY} \backslash 05-\mathrm{Nov}-2003 \backslash 0 \backslash \backslash \#$ B3PW91/6-311+G (3DF, 2P) GEOM=CHECK GUESS=READ SCF=TIGHT \#P GFINPUT IOP

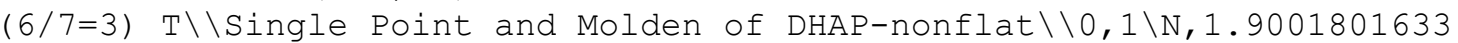
$, 0 ., 0.0625103116 \backslash \mathrm{C}, 1.1885684023,-1.1325461308,0.0411483997 \backslash \mathrm{C}, 1.1885684$ $023,1.1325461308,0.0411483997 \backslash \mathrm{C},-0.1978327664,-1.1961754089,-0.0030023$ $658 \backslash \mathrm{C},-0.1978327664,1.1961754089,-0.0030023658 \backslash \mathrm{C},-0.9329697689,0 .,-0.0$ $265298623 \backslash \mathrm{N},-2.311610154,0 .,-0.0208641789 \backslash \mathrm{H},-2.7669548945,0.8438000606$ $,-0.3387017251 \backslash \mathrm{H},-2.7669548945,-0.8438000606,-0.3387017251 \backslash \mathrm{H}, 1.7652340$ $18,-2.0570205185,0.0583535149 \backslash \mathrm{H}, 1.765234018,2.0570205185,0.0583535149 \backslash$ $\mathrm{H},-0.7037786647,-2.1587291867,-0.0146998705 \backslash \mathrm{H},-0.7037786647,2.15872918$ $67,-0.0146998705 \backslash \backslash$ Version=x86-Linux-G98RevA.11.1 $\backslash$ State $=1-A^{\prime} \backslash \mathrm{HF}=-303.63$ $13727 \backslash \mathrm{RMSD}=2.024 \mathrm{e}-09 \backslash \mathrm{Dipole}=-1.5312825,0 .,-0.370359 \backslash \mathrm{PG}=\mathrm{CS} \quad[\mathrm{SG}(\mathrm{C} 1 \mathrm{~N} 2), \mathrm{X}($ $\mathrm{C} 4 \mathrm{H} 6$ ) $] \backslash \backslash \mathrm{Q}$

\section{Single Point Energy of DHAP ligand, in $C_{S}$ symmetry}


$1 \backslash 1 \backslash G I N C-N E I L \backslash S P \backslash R M P 2-F C \backslash 6-31+G(d) \backslash C 5 H 6 N 2 \backslash C H U C K \backslash 02-A u g-2004 \backslash 0 \backslash \backslash \# M P 2 / 6$ $-31+\mathrm{G}(\mathrm{D}) \quad \mathrm{POP}=(\mathrm{NBOREAD}) \quad \mathrm{TEST} \backslash \backslash$ Hardness of $\mathrm{PMe} \backslash \backslash \backslash 0,1 \backslash \mathrm{N}, 0,0.001997,-1.901$ $207,0 . \backslash \mathrm{C}, 0,0.003296,-1.189276,1.132546 \backslash \mathrm{C}, 0,0.003296,-1.189276,-1.13254$ $6 \backslash \mathrm{C}, 0,0.003296,0.197828,1.196175 \backslash \mathrm{C}, 0,0.003296,0.197828,-1.196175 \backslash \mathrm{C}, 0,0$ $.00318,0.933341,0 . \backslash \mathrm{N}, 0,0.052724,2.311103,0 . \backslash \mathrm{H}, 0,-0.250459,2.776334,-0$. $8438 \backslash \mathrm{H}, 0,-0.250459,2.776334,0.8438 \backslash \mathrm{H}, 0,0.002138,-1.766197,2.057021 \backslash \mathrm{H}, 0$ $, 0.002138,-1.766197,-2.057021 \backslash \mathrm{H}, 0,0.007708,0.70389,2.158729 \backslash \mathrm{H}, 0,0.0077$ $08,0.70389,-2.158729 \backslash \backslash$ Version=x86-Linux-G98RevA.11.1 $\backslash$ State $=1-A^{\prime} \backslash H F=-30$ $1.7375863 \backslash \mathrm{MP} 2=-302.70019 \backslash \mathrm{RMSD}=3.384 \mathrm{e}-09 \backslash \mathrm{PG}=\mathrm{CS} \quad[\mathrm{SG}(\mathrm{C} 1 \mathrm{~N} 2), \mathrm{X}(\mathrm{C} 4 \mathrm{H} 6)] \backslash \backslash \mathrm{a}$

\section{Geometry Optimization of NHC ligand, in $C_{2 v}$ symmetry}

$1 \backslash 1 \backslash \mathrm{GINC}-\mathrm{NEIL} \backslash \mathrm{FOpt} \backslash \mathrm{RB} 3 \mathrm{PW} 91 \backslash 6-31 \mathrm{G}(\mathrm{d}) \backslash \mathrm{C} 3 \mathrm{H} 4 \mathrm{~N} 2 \backslash \mathrm{BOBBY} \backslash 12-\mathrm{Jan}-2003 \backslash 1 \backslash \backslash \#$ B3PW 91/6-31G(D) FOPT=Z-MATRIX FREQ=NORAMAN POP=(FULL, NBO) TEST \\Geom and $\mathrm{F}$ req of $\mathrm{H} 2-\mathrm{N}-\mathrm{Carbene} \backslash \backslash 0,1 \backslash \mathrm{C} \backslash \mathrm{X}, 1,1 . \backslash \mathrm{N}, 1, \mathrm{nc} 1,2, \mathrm{ncx} 1 \backslash \mathrm{N}, 1, \mathrm{nc} 1,2, \mathrm{ncx} 1,3,180$. $, 0 \backslash \mathrm{C}, 3, \mathrm{cn} 1,1, \mathrm{Cnc1}, 2,180 ., 0 \backslash \mathrm{C}, 4, \mathrm{cn} 1,1, \mathrm{cnc1}, 2,180 ., 0 \backslash \mathrm{H}, 5, \mathrm{hc} 1,3, \mathrm{hcn} 1,1,18$ $0 ., 0 \backslash \mathrm{H}, 6, h_{c 1}, 4, h \mathrm{cn} 1,1,180 ., 0 \backslash \mathrm{H}, 3, \mathrm{hc} 2,1, \mathrm{hcn} 2,2,0 ., 0 \backslash \mathrm{H}, 4, \mathrm{hc} 2,1, \mathrm{hcn} 2,2,0$. , $0 \backslash \backslash \mathrm{nc} 1=1.36792295 \backslash \mathrm{ncx} 1=130.10246281 \backslash \mathrm{cn} 1=1.38757762 \backslash \mathrm{cncl}=114.70006298 \backslash$ $\mathrm{hc1}=1.07987445 \backslash \mathrm{hcn} 1=123.69068709 \backslash \mathrm{hc} 2=1.00793673 \backslash \mathrm{hcn} 2=121.4121148 \backslash \backslash$ Vers ion=x86-Linux-G98RevA.11.1 \State=1-A1 \HF=-226.0829683 $\backslash \mathrm{RMSD}=1.494 \mathrm{e}-09 \backslash \mathrm{R}$ $\mathrm{MSF}=1.197 e-05 \backslash \mathrm{Dipole}=0 ., 0 .,-1.0596044 \backslash \mathrm{PG}=\mathrm{C} 02 \mathrm{~V} \quad[\mathrm{C} 2(\mathrm{C} 1), \mathrm{SGV}(\mathrm{C} 2 \mathrm{H} 4 \mathrm{~N} 2)] \backslash \backslash \mathrm{Q}$

\section{Single Point Energy of NHC ligand, in $C_{2 v}$ symmetry}

$1 \backslash 1 \backslash G I N C-N E I L \backslash S P \backslash R B 3 P W 91 \backslash 6-311+G(3 d f, 2 p) \backslash C 3 H 4 N 2 \backslash B O B B Y \backslash 09-F e b-2003 \backslash 0 \backslash \backslash \#$ B3PW91/6-311+G (3DF, 2P) GEOM=CHECK GUESS=READ SCF=TIGHT \#P GFINPUT IOP

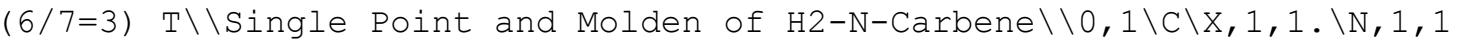
$.3679229491,2,130.10246281 \backslash \mathrm{N}, 1,1.3679229491,2,130.10246281,3,180 ., 0 \backslash \mathrm{C}$, $3,1.3875776183,1,114.70006298,2,180 ., 0 \backslash \mathrm{C}, 4,1.3875776183,1,114.70006298$ , 2, $180 ., 0 \backslash \mathrm{H}, 5,1.0798744524,3,123.69068709,1,180 ., 0 \backslash \mathrm{H}, 6,1.0798744524,4$, $123.69068709,1,180 ., 0 \backslash \mathrm{H}, 3,1.0079367284,1,121.4121148,2,0 ., 0 \backslash \mathrm{H}, 4,1.0079$ $367284,1,121.4121148,2,0$. ,0 \\Version=x86-Linux-G98RevA.11.1 \State=1-A1 $\backslash \mathrm{HF}=-226.1679042 \backslash \mathrm{RMSD}=8.536 \mathrm{e}-09 \backslash \mathrm{Dipole}=0,0 .,-1.1778078 \backslash \mathrm{PG}=\mathrm{C} 02 \mathrm{~V} \quad[\mathrm{C} 2(\mathrm{C} 1$ ), $\mathrm{SGV}(\mathrm{C} 2 \mathrm{H} 4 \mathrm{~N} 2)] \backslash \backslash \mathrm{Q}$

\section{MP2 Single Point Energy of NHC ligand, in $C_{2 v}$ symmetry}

$1 \backslash 1 \backslash G I N C-N E I L \backslash S P \backslash R M P 2-F C \backslash 6-31+G(d) \backslash C 3 H 4 N 2 \backslash C H U C K \backslash 02-A u g-2004 \backslash 0 \backslash \backslash \#$ MP2/6 $-31+\mathrm{G}(\mathrm{D}) \quad \mathrm{POP}=(\mathrm{NBOREAD}) \mathrm{TEST} \backslash \backslash$ Hardness of $\mathrm{H} 2-\mathrm{Carbene} \backslash \backslash 0,1 \backslash \mathrm{C}, 0,0,0 ., 1.2$ $82117 \backslash \mathrm{N}, 0,0.1 .046316,0.40096 \backslash \mathrm{N}, 0,0 .,-1.046316,0.40096 \backslash \mathrm{C}, 0,0 ., 0.67778$, $-0.936782 \backslash \mathrm{C}, 0,0 .,-0.67778,-0.936782 \backslash \mathrm{H}, 0,0.1 .384916,-1.752923 \backslash \mathrm{H}, 0,0 .,-$ $1.384916,-1.752923 \backslash \mathrm{H}, 0,0.2 .002247,0.72054 \backslash \mathrm{H}, 0,0 .,-2.002247,0.72054 \backslash \backslash \mathrm{V}$

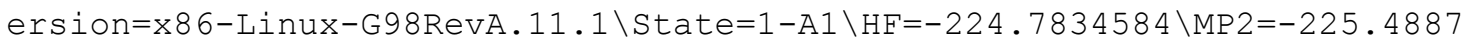
$121 \backslash \mathrm{RMSD}=4.678 \mathrm{e}-09 \backslash \mathrm{PG}=\mathrm{C} 02 \mathrm{~V} \quad[\mathrm{C} 2(\mathrm{C} 1), \mathrm{SGV}(\mathrm{C} 2 \mathrm{H} 4 \mathrm{~N} 2)] \backslash \backslash \mathrm{C}$

\begin{tabular}{|c|c|c|c|c|c|c|c|}
\hline Model & HOMO & LUMO & $\chi$ & $\eta$ & $-\Delta q(\mathbf{1})$ & $-\Delta q(2)$ & $-\Delta q\left(H^{-}\right)$ \\
\hline 1 & -13.11 & -5.09 & 9.10 & 4.01 & & & 0.77 \\
\hline 2 & -14.82 & -3.57 & 9.20 & 5.63 & & & 0.68 \\
\hline PMe3 & -9.00 & 1.59 & 3.70 & 5.29 & 0.58 & 0.50 & \\
\hline
\end{tabular}




\begin{tabular}{lllllll}
\hline DHAP & -8.99 & 1.71 & 3.64 & 5.35 & 0.58 & 0.51 \\
NHC & -8.86 & 1.90 & 3.48 & 5.38 & 0.60 & 0.52 \\
\hline
\end{tabular}

\section{COORDINATION COMPLEX CALCULATIONS}

\begin{tabular}{|c|c|c|c|c|c|c|c|}
\hline Model & Symm. & Energy (au) & $\begin{array}{c}\text { ZPVE } \\
\text { (au) }\end{array}$ & $\begin{array}{l}{ }^{(\text {a) }} \text { Corrected } \\
\text { Energy (au) }\end{array}$ & $\begin{array}{c}{ }^{(b)} \text { Cation } \\
\Delta \mathbf{H}_{\text {prep }} \\
\left(\mathrm{kJ} \mathrm{mol}^{-1}\right)\end{array}$ & $\begin{array}{c}{ }^{(\mathrm{c})} \mathrm{P}-\mathrm{E} \\
\text { Bond } \\
\text { Energy } \\
\left(\mathrm{kJ} \mathrm{mol}^{-1}\right) \\
\end{array}$ & $\begin{array}{c}{ }^{(\mathrm{d})} \Delta \mathrm{H}_{\mathrm{rxn}} \\
\left(\mathrm{kJ} \mathrm{mol}^{-1}\right)\end{array}$ \\
\hline 3-Ph & $\overline{C_{l}}$ & -1265.48274 & 0.30077 & -1265.18877 & 39.1 & 295.7 & -220.7 \\
\hline $3-\mathrm{NMe}_{2}$ & $C_{s}$ & -1071.36573 & 0.28461 & -1071.08756 & 134.0 & 251.7 & -91.2 \\
\hline 4-Ph & $C_{1}$ & -1108.02965 & 0.29344 & -1107.74284 & 46.4 & 283.0 & -217.7 \\
\hline $4-\mathrm{NMe}_{2}$ & $C_{1}$ & -913.92244 & 0.27813 & -913.65060 & 67.2 & 194.5 & -111.8 \\
\hline 5-Ph & $C_{1}$ & -1030.60151 & 0.25982 & -1030.34756 & 47.7 & 375.0 & -308.5 \\
\hline $5-\mathrm{NMe}_{2}$ & $C_{s}$ & -836.49054 & 0.24464 & -836.25143 & 165.0 & 374.6 & -192.3 \\
\hline
\end{tabular}

(a) Corrected energy: $\mathrm{E}_{\text {corrected }}=\mathrm{E}_{\text {calculated }}+0.9774 *$ ZPVE. (b) The preparation energy for the donor is calculated for the process: Acceptor $\rightarrow$ Acceptor* $; \Delta \mathrm{H}_{\text {prep }}=\mathrm{E}_{\text {acceptor* }}-\mathrm{E}_{\text {acceptor }}$, where the “*” indicates that the donor/acceptor fragment is in the exact same geometry observed in the coordination complex. (c) The P-E bond energy is calculated by: Complex $\rightarrow$ Donor* + Acceptor*; B.E. $=\left(E_{\text {donor* }}+E_{\text {acceptor* }}\right)-E_{\text {complex }}$ (d) For the reaction: Donor + Acceptor $\rightarrow$ Complex, $\Delta \mathrm{H}_{\mathrm{rxn}}=\mathrm{E}_{\text {complex }}-\left(\mathrm{E}_{\mathrm{donor}}+\mathrm{E}_{\text {acceptor }}\right)$.

Geometry Optimization of $\mathrm{Ph}_{2} \mathrm{P}-\mathrm{PMe}_{3}{ }^{+}$, 3-Ph, in $C_{1}$ symmetry

$1 \backslash 1 \backslash G I N C-N E I L \backslash F O p t \backslash R B 3 P W 91 \backslash 6-31 G(d) \backslash C 15 H 19 P 2(1+) \backslash B O B B Y \backslash 28-J a n-2003 \backslash 0 \backslash \backslash$ \# B3PW91/6-31G(D) OPT FREQ=NORAMAN POP=(FULL, NBO) TEST \\Geom and Freq of $\mathrm{Ph} 2 \mathrm{P}-\mathrm{PMe} 3+\backslash \backslash 1,1 \backslash \mathrm{P}, 0.0050893248,0.2281486628,-1.0406244714 \backslash \mathrm{C}, 1.43744$ $89407,-0.7281493143,-0.4037260058 \backslash C,-1.542589523,-0.5174474878,-0.4046$ $360969 \backslash \mathrm{C}, 2.6768047377,-0.4377663712,-1.0007378603 \backslash \mathrm{C},-2.4875501698,-0.8$ $287279019,-1.3961542984 \backslash C, 1.3588499022,-1.7569764544,0.5452315207 \backslash C,-1$ $.8557241797,-0.7820504797,0.9401704186 \backslash C, 3.8218776981,-1.1333287915,-0$ $.621080595 \backslash \mathrm{C},-3.7118095581,-1.3992332272,-1.0518507448 \backslash \mathrm{C}, 2.5056907346$, $-2.4600203696,0.9082454555 \backslash C,-3.0833042955,-1.3438429584,1.2797838433 \backslash$ $\mathrm{C}, 3.7375031269,-2.1432581537,0.3364941264 \backslash \mathrm{C},-4.0113048027,-1.653945094$ $, 0.284419964 \backslash \mathrm{H}, 2.7419742794,0.3123659344,-1.7864209854 \backslash \mathrm{H},-2.2620260383$ $,-0.6281871792,-2.440723811 \backslash \mathrm{H}, 0.4036450787,-2.035274945,0.9776323956 \backslash \mathrm{H}$ $,-1.1397638371,-0.567071178,1.7280841403 \backslash \mathrm{H}, 4.7740527109,-0.9021739147$, $-1.0900544054 \backslash \mathrm{H},-4.4304310427,-1.6419968369,-1.8291970426 \backslash \mathrm{H}, 2.43368370$ $99,-3.2637277265,1.6356805924 \backslash \mathrm{H},-3.3144677679,-1.5450938932,2.32213435$ $86 \backslash \mathrm{H}, 4.627416582,-2.6966112257,0.6225248768 \backslash \mathrm{H},-4.9667784668,-2.0953304$ $841,0.5531981383 \backslash \mathrm{P}, 0.1519158951,2.047291594,0.2296239139 \backslash \mathrm{C}, 0.426281226$ $7,1.8397498659,2.019639639 \backslash \mathrm{C}, 1.554867174,3.0189117446,-0.4011603586 \backslash \mathrm{C}$, $-1.3601654209,3.0259342619,-0.016166841 \backslash H, 1.2372436937,1.1244683087,2$. $1862226522 \backslash \mathrm{H}, 0.7021920621,2.8035472305,2.4609264952 \backslash \mathrm{H},-0.4826459394,1$. $4768151565,2.5049965438 \backslash \mathrm{H}, 1.4267515723,3.2092819806,-1.4705752228 \backslash \mathrm{H}, 1$. $6117220644,3.974182274,0.1305678972 \backslash H, 2.4878158587,2.4693807634,-0.244$ $9320365 \backslash \mathrm{H},-1.5010643492,3.2249509528,-1.0826384514 \backslash \mathrm{H},-2.2257146305,2.4$ $721902813,0.3579765346 \backslash H,-1.2799373852,3.977585037,0.5187726929 \backslash \backslash$ Versi on $=x 86-$ Linux - G98RevA. $11.1 \backslash \mathrm{HF}=-1265.2415447 \backslash \mathrm{RMSD}=7.705 \mathrm{e}-09 \backslash \mathrm{RMSF}=6.191 \mathrm{e}-$ 
$06 \backslash$ Dipole $=0.0031626,1.9664534,0.8146626 \backslash P G=C 01 \quad[X(C 15 H 19 P 2)] \backslash \backslash @$

\section{Single Point Energy Calculation of $\mathrm{Ph}_{2} \mathrm{P}-\mathrm{PMe}_{3}{ }^{+}, \mathbf{3}-\mathbf{P h}$, in $C_{1}$ symmetry}

$1 \backslash 1 \backslash G I N C-N E I L \backslash S P \backslash R B 3 P W 91 \backslash 6-311+G(3 d f, 2 p) \backslash C 15 H 19 P 2(1+) \backslash B O B B Y \backslash 01-$ Feb-200 $3 \backslash 0 \backslash \backslash \#$ B3PW91/6-311+G(3DF,2P) SCF=TIGHT \#P GFINPUT IOP(6/7=3) TEST \\i ngle Point and Molden of Ph2P-PMe3+\\1,1\P,0,0.005174,0.225498,-1.0412 $02 \backslash \mathrm{C}, 0,1.436418,-0.731043,-0.402163 \backslash \mathrm{C}, 0,-1.543344,-0.516465,-0.403011 \backslash$ $\mathrm{C}, 0,2.676029,-0.443791,-1.000159 \backslash \mathrm{C}, 0,-2.488911,-0.829033,-1.393546 \backslash \mathrm{C}, 0$ $, 1.356674,-1.757353,0.549421 \backslash \mathrm{C}, 0,-1.856548,-0.777244,0.942526 \backslash \mathrm{C}, 0,3.82$ $0273,-1.139876,-0.618966 \backslash \mathrm{C}, 0,-3.713841,-1.397068,-1.047548 \backslash \mathrm{C}, 0,2.50267$ $3,-2.460965,0.913989 \backslash \mathrm{C}, 0,-3.084789,-1.336574,1.283812 \backslash \mathrm{C}, 0,3.73478,-2.1$ $47259,0.341188 \backslash \mathrm{C}, 0,-4.013395,-1.647995,0.289425 \backslash \mathrm{H}, 0,2.742014,0.304258$, $-1.787759 \backslash \mathrm{H}, 0,-2.263339,-0.63144,-2.438666 \backslash \mathrm{H}, 0,0.401196,-2.033309,0.98$ $2719 \backslash \mathrm{H}, 0,-1.140148,-0.561196,1.729748 \backslash \mathrm{H}, 0,4.772653,-0.911153,-1.088716$ $\backslash \mathrm{H}, 0,-4.432936,-1.64087,-1.824131 \backslash \mathrm{H}, 0,2.429769,-3.262727,1.643478 \backslash \mathrm{H}, 0$, $-3.316002,-1.534875,2.326717 \backslash \mathrm{H}, 0,4.624031,-2.701042,0.628445 \backslash \mathrm{H}, 0,-4.96$ $9388,-2.087452,0.559515 \backslash \mathrm{P}, 0,0.154626,2.047669,0.224392 \backslash \mathrm{C}, 0,0.429086,1$. $844318,2.014874 \backslash \mathrm{C}, 0,1.558712,3.015857,-0.40914 \backslash \mathrm{C}, 0,-1.356231,3.027651$, $-0.023581 \backslash \mathrm{H}, 0,1.239151,1.128407,2.183111 \backslash \mathrm{H}, 0,0.706341,2.808873,2.45365$ $6 \backslash \mathrm{H}, 0,-0.480214,1.4838,2.501334 \backslash \mathrm{H}, 0,1.430626,3.203677,-1.479009 \backslash \mathrm{H}, 0,1$. $616918,3.9724,0.120149 \backslash \mathrm{H}, 0,2.490977,2.465511,-0.251703 \backslash \mathrm{H}, 0,-1.497088,3$ $.224142,-1.090526 \backslash \mathrm{H}, 0,-2.222423,2.475986,0.352142 \backslash \mathrm{H}, 0,-1.274655,3.9805$ $52,0.508923 \backslash \backslash$ Version=x86-Linux-G98RevA.11.1 \HF=-1265.4827442\RMSD=8.43 $9 e-09 \backslash \mathrm{Dipole}=0.0188338,1.9406828,0.7517904 \backslash \mathrm{PG}=\mathrm{C} 01 \quad[\mathrm{X}(\mathrm{C} 15 \mathrm{H} 19 \mathrm{P} 2)] \backslash \backslash \mathrm{C}$

\section{Single Point Energy Calculation of the $\mathrm{Ph}_{2} \mathrm{P}^{+}$Fragment from $\mathrm{Ph}_{2} \mathrm{P}-\mathrm{PMe}_{3}{ }^{+}, \mathbf{3}-\mathbf{P h}$, in $C_{1}$ symmetry}

$1 \backslash 1 \backslash G I N C-N E I L \backslash S P \backslash R B 3 P W 91 \backslash 6-311+G(3 d f, 2 p) \backslash C 12 H 10 P 1(1+) \backslash B O B B Y \backslash 02-$ Feb-200 $3 \backslash 0 \backslash \backslash \#$ B3PW91/6-311+G(3DF,2P) SCF=TIGHT \#P GFINPUT IOP (6/7=3) TEST \\i

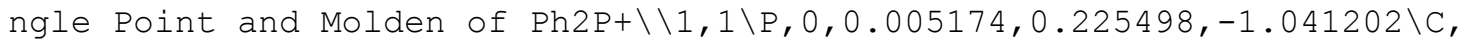
$0,1.436418,-0.731043,-0.402163 \backslash \mathrm{C}, 0,-1.543344,-0.516465,-0.403011 \backslash \mathrm{C}, 0,2$ $.676029,-0.443791,-1.000159 \backslash \mathrm{C}, 0,-2.488911,-0.829033,-1.393546 \backslash \mathrm{C}, 0,1.35$ $6674,-1.757353,0.549421 \backslash \mathrm{C}, 0,-1.856548,-0.777244,0.942526 \backslash \mathrm{C}, 0,3.820273$, $-1.139876,-0.618966 \backslash \mathrm{C}, 0,-3.713841,-1.397068,-1.047548 \backslash \mathrm{C}, 0,2.502673,-2$. $460965,0.913989 \backslash \mathrm{C}, 0,-3.084789,-1.336574,1.283812 \backslash \mathrm{C}, 0,3.73478,-2.147259$ $, 0.341188 \backslash \mathrm{C}, 0,-4.013395,-1.647995,0.289425 \backslash \mathrm{H}, 0,2.742014,0.304258,-1.78$ $7759 \backslash \mathrm{H}, 0,-2.263339,-0.63144,-2.438666 \backslash \mathrm{H}, 0,0.401196,-2.033309,0.982719 \backslash$ $\mathrm{H}, 0,-1.140148,-0.561196,1.729748 \backslash \mathrm{H}, 0,4.772653,-0.911153,-1.088716 \backslash \mathrm{H}, 0$, $-4.432936,-1.64087,-1.824131 \backslash \mathrm{H}, 0,2.429769,-3.262727,1.643478 \backslash \mathrm{H}, 0,-3.31$ $6002,-1.534875,2.326717 \backslash \mathrm{H}, 0,4.624031,-2.701042,0.628445 \backslash \mathrm{H}, 0,-4.969388$, $-2.087452,0.559515 \backslash \backslash$ Version=x86-Linux-G98RevA.11.1 \HF=-804.2971751 $\backslash$ RMS $\mathrm{D}=7.159 e-09 \backslash \mathrm{Dipole}=0.4668572,-0.2605169,0.1037246 \backslash \mathrm{PG}=\mathrm{C} 01 \quad[\mathrm{X}(\mathrm{C} 12 \mathrm{H} 10 \mathrm{P} 1)]$ $\backslash \backslash Q$

\section{Single Point Energy Calculation of the $\mathrm{PMe}_{3}$ Fragment from $\mathrm{Ph}_{2} \mathrm{P}-\mathrm{PMe}_{3}{ }^{+}, \mathbf{3}-\mathbf{P h}$, in $C_{1}$ symmetry}

$1 \backslash 1 \backslash G I N C-N E I L \backslash S P \backslash R B 3 P W 91 \backslash 6-311+G(3 d f, 2 p) \backslash C 3 H 9 P 1 \backslash B O B B Y \backslash 02-F e b-2003 \backslash 0 \backslash \backslash \#$ B3PW91/6-311+G(3DF,2P) SCF=TIGHT \#P GFINPUT IOP(6/7=3) TEST \Single P 


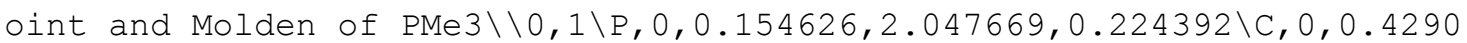
$86,1.844318,2.014874 \backslash \mathrm{C}, 0,1.558712,3.015857,-0.40914 \backslash \mathrm{C}, 0,-1.356231,3.02$ $7651,-0.023581 \backslash \mathrm{H}, 0,1.239151,1.128407,2.183111 \backslash \mathrm{H}, 0,0.706341,2.808873,2$. $453656 \backslash \mathrm{H}, 0,-0.480214,1.4838,2.501334 \backslash \mathrm{H}, 0,1.430626,3.203677,-1.479009 \backslash \mathrm{H}$ $, 0,1.616918,3.9724,0.120149 \backslash \mathrm{H}, 0,2.490977,2.465511,-0.251703 \backslash \mathrm{H}, 0,-1.497$ $088,3.224142,-1.090526 \backslash \mathrm{H}, 0,-2.222423,2.475986,0.352142 \backslash \mathrm{H}, 0,-1.274655,3$ $.980552,0.508923 \backslash \backslash$ Version=x86-Linux-G98RevA.11.1 \HF=-461.0729395 RMSD= $2.313 e-09 \backslash \mathrm{Dipole}=0.0362637,0.5417354,0.2778807 \backslash \mathrm{PG}=\mathrm{C} 01 \quad[\mathrm{X}(\mathrm{C} 3 \mathrm{H} 9 \mathrm{P} 1)] \backslash \backslash \mathrm{Q}$

\section{Geometry Optimization of $\left(\mathrm{Me}_{2} \mathrm{~N}\right)_{2} \mathrm{P}-\mathrm{PMe}_{3}{ }^{+}, \mathbf{3}-\mathrm{NMe}_{2}$, in $C_{s}$ symmetry}

$1 \backslash 1 \backslash G I N C-N E I L \backslash F O p t \backslash R B 3 P W 91 \backslash 6-31 G(d) \backslash C 7 H 21 N 2 P 2(1+) \backslash B O B B Y \backslash 22-J a n-2003 \backslash 0 \backslash$ $\backslash \#$ B3PW91/6-31G(D) OPT FREQ=NORAMAN POP=(FULL, NBO) TEST \\Geom and Freq of $(\mathrm{Me} 2 \mathrm{~N}) 2 \mathrm{P}-\mathrm{PMe} 3+-\mathrm{Cs} \backslash \backslash 1,1 \backslash \mathrm{P}, 0.6444606411,0,-0.6712043834 \backslash \mathrm{P}, 0.2365692$ $653,0.1 .544469561 \backslash \mathrm{N},-0.1318002107,-1.4306781368,-1.1276737498 \backslash \mathrm{N},-0.13$ $18002107,1.4306781368,-1.1276737498 \backslash \mathrm{C}, 0.6146731634,-2.3010104619,-2.04$ $56054661 \backslash \mathrm{C}, 0.6146731634,2.3010104619,-2.0456054661 \backslash \mathrm{C},-1.565190507,-1.6$ $998882111,-1.1313814744 \backslash \mathrm{C},-1.565190507,1.6998882111,-1.1313814744 \backslash \mathrm{H}, 1$. $6811717533,-2.0651413324,-2.0082700555 \backslash \mathrm{H}, 1.6811717533,2.0651413324,-2$. $0082700555 \backslash \mathrm{H},-1.7374193389,-2.7657796635,-0.9393117686 \backslash \mathrm{H},-1.7374193389$ $, 2.7657796635,-0.9393117686 \backslash \mathrm{H}, 0.4814556761,-3.3475860445,-1.7482050441$ $\backslash \mathrm{H}, 0.2665878665,2.1869451645,-3.0808985461 \backslash \mathrm{H}, 0.2665878665,-2.186945164$ $5,-3.0808985461 \backslash \mathrm{H}, 0.4814556761,3.3475860445,-1.7482050441 \backslash \mathrm{H},-2.0756649$ $104,-1.1294346536,-0.3525562352 \backslash \mathrm{H},-2.0269779685,1.4513269273,-2.096739$ $2003 \backslash \mathrm{H},-2.0269779685,-1.4513269273,-2.0967392003 \backslash \mathrm{H},-2.0756649104,1.129$ $4346536,-0.3525562352 \backslash \mathrm{C},-1.4320733812,0.2 .2950421422 \backslash \mathrm{C}, 1.0940594194,1$ $.4713398954,2.1860622114 \backslash \mathrm{C}, 1.0940594194,-1.4713398954,2.1860622114 \backslash \mathrm{H}, 2$ $.1353279814,1.4678084951,1.8506829322 \backslash \mathrm{H}, 2.1353279814,-1.4678084951,1.8$ $506829322 \backslash \mathrm{H},-1.3282105113,0.3 .3860114955 \backslash \mathrm{H}, 0.60791358,2.375083949,1.8$ $08083243 \backslash \mathrm{H}, 1.0706324238,1.4777385531,3.2803082175 \backslash \mathrm{H}, 1.0706324238,-1.47$ $77385531,3.2803082175 \backslash \mathrm{H}, 0.60791358,-2.375083949,1.808083243 \backslash \mathrm{H},-1.98907$ $69424,0.8924442482,1.9985500736 \backslash \mathrm{H},-1.9890769424,-0.8924442482,1.998550$ $0736 \backslash \backslash$ Version=x86-Linux-G98RevA.11.1 \State=1-A ' $\backslash \mathrm{HF}=-1071.1637903 \backslash \mathrm{RMSD}=$ $9.431 e-09 \backslash \mathrm{RMSF}=9.524 \mathrm{e}-06 \backslash \mathrm{Dipole}=-0.3043346,0 ., 1.1918288 \backslash \mathrm{PG}=\mathrm{CS} \quad[\mathrm{SG}(\mathrm{C} 1 \mathrm{H} 1$ $\mathrm{P} 2), \mathrm{X}(\mathrm{C} 6 \mathrm{H} 2 \mathrm{ON} 2)] \backslash \backslash \mathrm{Q}$

\section{Single Point Energy Calculation of $\left(\mathrm{Me}_{2} \mathrm{~N}\right)_{2} \mathrm{P}-\mathrm{PMe}_{3}{ }^{+}, \mathbf{3}-\mathrm{NMe}_{2}$, in $C_{s}$ symmetry}

$1 \backslash 1 \backslash G I N C-N E I L \backslash S P \backslash R B 3 P W 91 \backslash 6-311+G(3 d f, 2 p) \backslash C 7 H 21 N 2 P 2(1+) \backslash B O B B Y \backslash 28-J a n-20$ $03 \backslash 0 \backslash \backslash \#$ B3PW91/6-311+G(3DF, 2P) SCF=TIGHT \#P GFINPUT IOP (6/7=3) TEST \\S ingle Point and Molden of (Me2N)2P-PMe3+-Cs \\1,1 $1 \mathrm{P}, 0,0 ., 0.930508,0 . \backslash \mathrm{P}$, $0,-1.240329,-0.950229,0 . \backslash N, 0,0.876087,0.722143,1.430678 \backslash \mathrm{N}, 0,0.876087,0$ $.722143,-1.430678 \backslash \mathrm{C}, 0,0.973383,1.901276,2.30101 \backslash \mathrm{C}, 0,0.973383,1.901276$, $-2.30101 \backslash \mathrm{C}, 0,1.912605,-0.267935,1.699888 \backslash \mathrm{C}, 0,1.912605,-0.267935,-1.699$ $888 \backslash \mathrm{H}, 0,0.178227,2.612991,2.065141 \backslash \mathrm{H}, 0,0.178227,2.612991,-2.065141 \backslash \mathrm{H}, 0$ $, 1.903813,-0.525765,2.76578 \backslash \mathrm{H}, 0,1.903813,-0.525765,-2.76578 \backslash \mathrm{H}, 0,0.8635$ $01,1.594486,3.347586 \backslash \mathrm{H}, 0,1.941502,2.406985,-2.186945 \backslash \mathrm{H}, 0,1.941502,2.40$ $6985,2.186945 \backslash \mathrm{H}, 0,0.863501,1.594486,-3.347586 \backslash \mathrm{H}, 0,1.741419,-1.183275,1$ $.129435 \backslash \mathrm{H}, 0,2.914304,0.108578,-1.451327 \backslash \mathrm{H}, 0,2.914304,0.108578,1.451327$ $\backslash \mathrm{H}, 0,1.741419,-1.183275,-1.129435 \backslash \mathrm{C}, 0,-0.556524,-2.647326,0 . \backslash \mathrm{C}, 0,-2.30$ $3225,-0.81914,-1.47134 \backslash \mathrm{C}, 0,-2.303225,-0.81914,1.47134 \backslash \mathrm{H}, 0,-2.822044,0$. $143952,-1.467808 \backslash \mathrm{H}, 0,-2.822044,0.143952,1.467808 \backslash \mathrm{H}, 0,-1.387039,-3.3623$ $42,0 . \backslash \mathrm{H}, 0,-1.690768,-0.883192,-2.375084 \backslash \mathrm{H}, 0,-3.04419,-1.624679,-1.4777$ 
$39 \backslash \mathrm{H}, 0,-3.04419,-1.624679,1.477739 \backslash \mathrm{H}, 0,-1.690768,-0.883192,2.375084 \backslash \mathrm{H}$, $0,0.050607,-2.819232,-0.892444 \backslash \mathrm{H}, 0,0.050607,-2.819232,0.892444 \backslash \backslash$ Versio $\mathrm{n}=\mathrm{x} 86-\mathrm{Linux}-\mathrm{G} 98 \mathrm{RevA} .11 .1 \backslash$ State $=1-\mathrm{A}^{\prime} \backslash \mathrm{HF}=-1071.3657312 \backslash \mathrm{RMSD}=2.858 \mathrm{e}-09 \backslash \mathrm{Di}$ pole $=-0.6198967,-1.0514745,0 . \backslash P G=C S \quad[S G(C 1 H 1 P 2), X(C 6 H 20 N 2)] \backslash \backslash @$

\section{Single Point Energy Calculation of the $\left(\mathrm{Me}_{2} \mathrm{~N}\right)_{2} \mathrm{P}^{+}$Fragment from $\left(\mathrm{Me}_{2} \mathrm{~N}\right)_{2} \mathrm{P}-\mathrm{PMe}_{3}{ }^{+}, 3-$ $\mathbf{N M e}_{2}$, in $C_{s}$ symmetry}

$1 \backslash 1 \backslash G I N C-N E I L \backslash S P \backslash R B 3 P W 91 \backslash 6-311+G(3 \mathrm{df}, 2 \mathrm{p}) \backslash \mathrm{C} 4 \mathrm{H} 12 \mathrm{~N} 2 \mathrm{P} 1(1+) \backslash \mathrm{BOBBY} \backslash 28-\mathrm{Jan}-20$ $03 \backslash 0 \backslash \backslash \#$ B3PW91/6-311+G(3DF, 2P) SCF=TIGHT \#P GFINPUT IOP $(6 / 7=3)$ TEST \\S

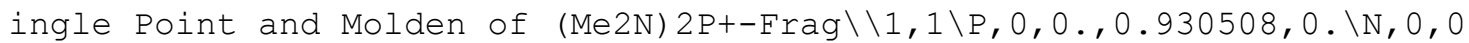
$.876087,0.722143,1.430678 \backslash \mathrm{N}, 0,0.876087,0.722143,-1.430678 \backslash \mathrm{C}, 0,0.973383$ $, 1.901276,2.30101 \backslash \mathrm{C}, 0,0.973383,1.901276,-2.30101 \backslash \mathrm{C}, 0,1.912605,-0.26793$ $5,1.699888 \backslash \mathrm{C}, 0,1.912605,-0.267935,-1.699888 \backslash \mathrm{H}, 0,0.178227,2.612991,2.06$ $5141 \backslash \mathrm{H}, 0,0.178227,2.612991,-2.065141 \backslash \mathrm{H}, 0,1.903813,-0.525765,2.76578 \backslash \mathrm{H}$, $0,1.903813,-0.525765,-2.76578 \backslash \mathrm{H}, 0,0.863501,1.594486,3.347586 \backslash \mathrm{H}, 0,1.941$ $502,2.406985,-2.186945 \backslash \mathrm{H}, 0,1.941502,2.406985,2.186945 \backslash \mathrm{H}, 0,0.863501,1.5$ $94486,-3.347586 \backslash \mathrm{H}, 0,1.741419,-1.183275,1.129435 \backslash \mathrm{H}, 0,2.914304,0.108578$, $-1.451327 \backslash \mathrm{H}, 0,2.914304,0.108578,1.451327 \backslash \mathrm{H}, 0,1.741419,-1.183275,-1.129$ $435 \backslash \backslash$ Version=x86-Linux-G98RevA.11.1 \State=1-A ' $\backslash H F=-610.1951531 \backslash R M S D=4$. $417 e-09 \backslash$ Dipole=0.4694887,0.0428745,0.\PG=CS [SG(P1),X (C4H12N2)] \\

\section{Single Point Energy Calculation of the $\mathrm{PMe}_{3}$ Fragment from $\left(\mathrm{Me}_{2} \mathrm{~N}\right)_{2} \mathrm{P}-\mathrm{PMe}_{3}{ }^{+}, \mathbf{3}-\mathrm{NMe}_{2}$, in $C_{s}$ symmetry}

$1 \backslash 1 \backslash G I N C-N E I L \backslash S P \backslash R B 3 P W 91 \backslash 6-311+G(3 d f, 2 p) \backslash C 3 H 9 P 1 \backslash B O B B Y \backslash 28-J a n-2003 \backslash 0 \backslash \backslash \#$ B3PW91/6-311+G(3DF,2P) SCF=TIGHT \#P GFINPUT IOP(6/7=3) TEST \Single P

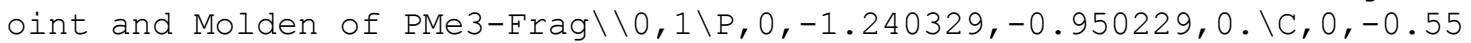
$6524,-2.647326,0 . \backslash \mathrm{C}, 0,-2.303225,-0.81914,-1.47134 \backslash \mathrm{C}, 0,-2.303225,-0.819$ $14,1.47134 \backslash \mathrm{H}, 0,-2.822044,0.143952,-1.467808 \backslash \mathrm{H}, 0,-2.822044,0.143952,1.4$ $67808 \backslash \mathrm{H}, 0,-1.387039,-3.362342,0 . \backslash \mathrm{H}, 0,-1.690768,-0.883192,-2.375084 \backslash \mathrm{H}, 0$ $,-3.04419,-1.624679,-1.477739 \backslash \mathrm{H}, 0,-3.04419,-1.624679,1.477739 \backslash \mathrm{H}, 0,-1.6$ $90768,-0.883192,2.375084 \backslash \mathrm{H}, 0,0.050607,-2.819232,-0.892444 \backslash \mathrm{H}, 0,0.050607$ $,-2.819232,0.892444 \backslash \backslash$ Version=x86-Linux-G98RevA.11. $1 \backslash$ State $=1-A^{\prime} \backslash H F=-461$ $.074719 \backslash \mathrm{RMSD}=2.143 e-09 \backslash \mathrm{Dipole}=-0.4437258,-0.4132123,0 . \backslash \mathrm{PG}=\mathrm{CS} \quad[\mathrm{SG}(\mathrm{C} 1 \mathrm{H} 1 \mathrm{P}$ 1), $\mathrm{X}(\mathrm{C} 2 \mathrm{H} 8)] \backslash \backslash \mathrm{Q}$

\section{Geometry Optimization of $\mathrm{Ph}_{2} \mathrm{P}-\mathrm{DHAP}{ }^{+}, \mathbf{4 - P h}$, in $C_{l}$ symmetry}

$1 \backslash 1 \backslash G I N C-N E I L \backslash F O p t \backslash R B 3 P W 91 \backslash 6-31 G(d) \backslash C 17 H 16 N 2 P 1(1+) \backslash B O B B Y \backslash 01-N o v-2003 \backslash 0$ $\backslash \backslash \#$ B3PW91/6-31G(D) OPT FREQ=NORAMAN POP=(FULL, NBO) TEST \\Geom and Fre $\mathrm{q}$ of $\mathrm{Ph} 2 \mathrm{P}-\mathrm{DHAP}+\backslash \backslash 1,1 \backslash \mathrm{P}, 0.2015414313,0.9287243521,0.9302467561 \backslash \mathrm{C}, 1.6329$ $652452,0.970580736,-0.1991202286 \backslash \mathrm{C},-1.2390489769,1.4172679722,-0.07878$ $63392 \backslash \mathrm{C}, 2.8983938498,0.8615169998,0.4047390353 \backslash \mathrm{C},-1.7854177156,2.67010$ $77676,0.2388988947 \backslash \mathrm{C}, 1.5479416434,1.1835864665,-1.5838956457 \backslash \mathrm{C},-1.8203$ $998103,0.655138928,-1.1036461776 \backslash \mathrm{C}, 4.0537781084,0.9280424034,-0.367653$ $8157 \backslash \mathrm{C},-2.8753879851,3.1642088208,-0.4750744359 \backslash \mathrm{C}, 2.7090258466,1.26056$ $12575,-2.3499980032 \backslash \mathrm{C},-2.9197905597,1.1454037624,-1.8023397356 \backslash \mathrm{C}, 3.958$ $9497575,1.1276820426,-1.7452642621 \backslash \mathrm{C},-3.4438811967,2.4017495971,-1.492$ $9354465 \backslash \mathrm{H}, 2.9776760132,0.736017873,1.482691377 \backslash \mathrm{H},-1.3594984819,3.26021$ $08286,1.0476539348 \backslash \mathrm{H}, 0.5792985566,1.3082215482,-2.05947997 \backslash \mathrm{H},-1.425557$ 
$9301,-0.3255554139,-1.3576790926 \backslash \mathrm{H}, 5.027356111,0.8420733097,0.10636197$ $01 \backslash \mathrm{H},-3.2866600744,4.1381989929,-0.2262149009 \backslash \mathrm{H}, 2.6373632501,1.4341342$ $884,-3.420070353 \backslash \mathrm{H},-3.3684300081,0.5478577307,-2.591161681 \backslash \mathrm{H}, 4.8614915$ $867,1.1949263758,-2.3463627143 \backslash \mathrm{H},-4.3010198653,2.7810843647,-2.0421006$ $723 \backslash \mathrm{N},-0.0850657446,-0.9059051557,1.0053243556 \backslash \mathrm{C},-0.8594347136,-1.3512$ $735141,2.0290764889 \backslash \mathrm{C}, 0.4379669805,-1.811303851,0.1413435907 \backslash \mathrm{C},-1.1446$ $965905,-2.6776363443,2.2094171387 \backslash \mathrm{C}, 0.2034779509,-3.1562716617,0.26428$ $81056 \backslash \mathrm{C},-0.6112146206,-3.6416288104,1.3171588768 \backslash \mathrm{H},-1.2382725164,-0.58$ $98111032,2.7032990289 \backslash \mathrm{H}, 1.0624993039,-1.409145072,-0.6485542333 \backslash \mathrm{H},-1.7$ $68859637,-2.9763085863,3.0453202587 \backslash \mathrm{H}, 0.6509776214,-3.8378262256,-0.45$ $20701782 \backslash \mathrm{N},-0.8612318814,-4.9498294792,1.4669802041 \backslash \mathrm{H},-1.4357981103,-5$ $.2940935688,2.2220306396 \backslash \mathrm{H},-0.4709671875,-5.6371036145,0.8392550821 \backslash \backslash \mathrm{V}$

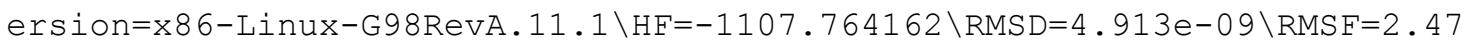
$0 e-06 \backslash$ Dipole $=-0.4938663,-3.1702282,0.6847849 \backslash \mathrm{PG}=\mathrm{C} 01 \quad[\mathrm{X}(\mathrm{C} 17 \mathrm{H} 16 \mathrm{~N} 2 \mathrm{P} 1)] \backslash \backslash \mathrm{C}$

\section{Single Point Energy Calculation of $\mathrm{Ph}_{2} \mathrm{P}-\mathrm{DHAP}{ }^{+}, \mathbf{4 - P h}$, in $C_{1}$ symmetry}

$1 \backslash 1 \backslash \mathrm{GINC}-\mathrm{NEIL} \backslash \mathrm{SP} \backslash \mathrm{RB} 3 \mathrm{PW} 91 \backslash 6-311+\mathrm{G}(3 \mathrm{df}, 2 \mathrm{p}) \backslash \mathrm{C} 17 \mathrm{H} 16 \mathrm{~N} 2 \mathrm{P} 1(1+) \backslash \mathrm{BOBBY} \backslash 06-\mathrm{Nov}-2$ $003 \backslash 0 \backslash \backslash \#$ B3PW91/6-311+G(3DF, 2P) SCF=TIGHT \#P GFINPUT IOP $(6 / 7=3)$ TEST \ Single Point and Molden of Ph2P-DHPAP+\\1,1\P,0,0.479019,-0.006406,-1. $240567 \backslash \mathrm{C}, 0,1.176195,1.451918,-0.395918 \backslash \mathrm{C}, 0,1.142139,-1.456586,-0.3521 \backslash$ $\mathrm{C}, 0,0.998718,2.685498,-1.047556 \backslash \mathrm{C}, 0,2.065564,-2.223417,-1.078947 \backslash \mathrm{C}, 0,1$ $.928947,1.401558,0.787735 \backslash \mathrm{C}, 0,0.801133,-1.847558,0.951741 \backslash \mathrm{C}, 0,1.533406$ $, 3.850033,-0.505433 \backslash \mathrm{C}, 0,2.658928,-3.344306,-0.501489 \backslash \mathrm{C}, 0,2.471212,2.56$ $9608,1.3193 \backslash \mathrm{C}, 0,1.383458,-2.977833,1.51793 \backslash \mathrm{C}, 0,2.269839,3.791938,0.678$ $184 \backslash \mathrm{C}, 0,2.316462,-3.72292,0.794635 \backslash \mathrm{H}, 0,0.450819,2.73073,-1.986586 \backslash \mathrm{H}, 0$, $2.320047,-1.945285,-2.099532 \backslash \mathrm{H}, 0,2.107376,0.450963,1.282219 \backslash \mathrm{H}, 0,0.0760$ $9,-1.279354,1.529366 \backslash \mathrm{H}, 0,1.391111,4.798984,-1.014529 \backslash \mathrm{H}, 0,3.37786,-3.92$ $728,-1.06983 \backslash \mathrm{H}, 0,3.060174,2.523299,2.231055 \backslash \mathrm{H}, 0,1.110975,-3.278059,2.5$ $25995 \backslash \mathrm{H}, 0,2.700338,4.698915,1.093499 \backslash \mathrm{H}, 0,2.769691,-4.603421,1.24125 \backslash \mathrm{N}$, $0,-1.242818,0.019926,-0.541833 \backslash \mathrm{C}, 0,-2.17129,-0.717803,-1.204981 \backslash \mathrm{C}, 0,-1$ $.630687,0.732115,0.545419 \backslash \mathrm{C}, 0,-3.478721,-0.781916,-0.80542 \backslash \mathrm{C}, 0,-2.9243$ $15,0.724018,0.99874 \backslash \mathrm{C}, 0,-3.906263,-0.048054,0.329773 \backslash \mathrm{H}, 0,-1.815204,-1$. $254061,-2.078794 \backslash \mathrm{H}, 0,-0.857957,1.31799,1.030548 \backslash \mathrm{H}, 0,-4.177218,-1.38734$ $6,-1.373815 \backslash \mathrm{H}, 0,-3.181779,1.316369,1.870963 \backslash \mathrm{N}, 0,-5.179979,-0.079039,0$. $745744 \backslash \mathrm{H}, 0,-5.879441,-0.62384,0.263365 \backslash \mathrm{H}, 0,-5.486933,0.453507,1.546285$ $\backslash \backslash$ Version=x86-Linux-G98RevA.11.1\HF=-1108.0296496\RMSD=4.941e-09\Dipol $e=-3.1072064,0.0253062,0.6517788 \backslash P G=C 01 \quad[X(C 17 H 16 \mathrm{~N} 2 \mathrm{P} 1)] \backslash \backslash @$

\section{Single Point Energy Calculation of the $\mathrm{Ph}_{2} \mathrm{P}^{+}$Fragment from $\mathrm{Ph}_{2} \mathrm{P}-\mathrm{DHAP}{ }^{+}, \mathbf{4 - P h}$, in $C_{1}$ symmetry}

$1 \backslash 1 \backslash G I N C-N E I L \backslash S P \backslash R B 3 P W 91 \backslash 6-311+G(3 d f, 2 p) \backslash C 12 H 10 P 1(1+) \backslash B O B B Y \backslash 05-N o v-200$ $3 \backslash 0 \backslash \backslash \#$ B3PW91/6-311+G(3DF,2P) SCF=TIGHT \#P GFINPUT IOP $(6 / 7=3)$ TEST $\backslash \mathrm{Si}$ ngle Point and Molden of Ph2P+-Frag \\1,1\P,0,0.479019,-0.006406,-1.240 $567 \backslash \mathrm{C}, 0,1.176195,1.451918,-0.395918 \backslash \mathrm{C}, 0,1.142139,-1.456586,-0.3521 \backslash \mathrm{C}, 0$ $, 0.998718,2.685498,-1.047556 \backslash \mathrm{C}, 0,2.065564,-2.223417,-1.078947 \backslash \mathrm{C}, 0,1.92$ $8947,1.401558,0.787735 \backslash \mathrm{C}, 0,0.801133,-1.847558,0.951741 \backslash \mathrm{C}, 0,1.533406,3$. $850033,-0.505433 \backslash \mathrm{C}, 0,2.658928,-3.344306,-0.501489 \backslash \mathrm{C}, 0,2.471212,2.56960$ $8,1.3193 \backslash \mathrm{C}, 0,1.383458,-2.977833,1.51793 \backslash \mathrm{C}, 0,2.269839,3.791938,0.678184$ $\backslash \mathrm{C}, 0,2.316462,-3.72292,0.794635 \backslash \mathrm{H}, 0,0.450819,2.73073,-1.986586 \backslash \mathrm{H}, 0,2.3$ $20047,-1.945285,-2.099532 \backslash \mathrm{H}, 0,2.107376,0.450963,1.282219 \backslash \mathrm{H}, 0,0.07609,-$ $1.279354,1.529366 \backslash \mathrm{H}, 0,1.391111,4.798984,-1.014529 \backslash \mathrm{H}, 0,3.37786,-3.92728$ 
$,-1.06983 \backslash \mathrm{H}, 0,3.060174,2.523299,2.231055 \backslash \mathrm{H}, 0,1.110975,-3.278059,2.5259$ $95 \backslash \mathrm{H}, 0,2.700338,4.698915,1.093499 \backslash \mathrm{H}, 0,2.769691,-4.603421,1.24125 \backslash \backslash$ Vers ion $=x 86-$ Linux-G98RevA.11. $1 \backslash H F=-804.2943917 \backslash R M S D=7.351 e-09 \backslash D i p o l e=0.141$ $0063,0.8453225,0.1198479 \backslash \mathrm{PG}=\mathrm{C} 01 \quad[\mathrm{X}(\mathrm{C} 12 \mathrm{H} 10 \mathrm{P} 1)] \backslash \backslash \mathrm{Q}$

\section{Single Point Energy Calculation of the DHAP Fragment from $\mathrm{Ph}_{2} \mathrm{P}-\mathrm{DHAP}^{+}, \mathbf{4 - P h}$, in $C_{l}$ symmetry}

$1 \backslash 1 \backslash G I N C-N E I L \backslash S P \backslash R B 3 P W 91 \backslash 6-311+G(3 d f, 2 p) \backslash C 5 H 6 N 2 \backslash B O B B Y \backslash 06-N o v-2003 \backslash 0 \backslash \backslash \#$ B3PW91/6-311+G(3DF, 2P) SCF=TIGHT \#P GFINPUT IOP(6/7=3) TEST \\Single P

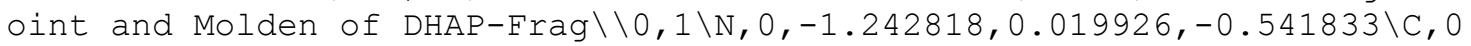
$,-2.17129,-0.717803,-1.204981 \backslash \mathrm{C}, 0,-1.630687,0.732115,0.545419 \backslash \mathrm{C}, 0,-3.4$ $78721,-0.781916,-0.80542 \backslash \mathrm{C}, 0,-2.924315,0.724018,0.99874 \backslash \mathrm{C}, 0,-3.906263$, $-0.048054,0.329773 \backslash \mathrm{H}, 0,-1.815204,-1.254061,-2.078794 \backslash \mathrm{H}, 0,-0.857957,1.3$ $1799,1.030548 \backslash \mathrm{H}, 0,-4.177218,-1.387346,-1.373815 \backslash \mathrm{H}, 0,-3.181779,1.316369$ $, 1.870963 \backslash \mathrm{N}, 0,-5.179979,-0.079039,0.745744 \backslash \mathrm{H}, 0,-5.879441,-0.62384,0.26$ $3365 \backslash \mathrm{H}, 0,-5.486933,0.453507,1.546285 \backslash \backslash$ Version=x86-Linux-G98RevA.11.1\H $\mathrm{F}=-303.6274634 \backslash \mathrm{RMSD}=3.291 \mathrm{e}-09 \backslash \mathrm{Dipole}=-1.7040082,-0.0272737,0.5438798 \backslash \mathrm{P}$ $\mathrm{G}=\mathrm{C} 01 \quad[\mathrm{X}(\mathrm{C} 5 \mathrm{H} 6 \mathrm{~N} 2)] \backslash \backslash @$

\section{Geometry Optimization of $\left(\mathrm{Me}_{2} \mathrm{~N}\right)_{2} \mathrm{P}-\mathrm{DHAP}^{+}, \mathbf{4}-\mathrm{NMe}_{2}$, in $C_{1}$ symmetry}

$1 \backslash 1 \backslash G I N C-N E I L \backslash F O p t \backslash R B 3 P W 91 \backslash 6-31 G(d) \backslash C 9 H 18 N 4 P 1(1+) \backslash B O B B Y \backslash 29-O c t-2003 \backslash 0 \backslash$ \\# B3PW91/6-31G(D) OPT FREQ=NORAMAN POP=(FULL, NBO) TEST \Geom and Freq of $(\mathrm{Me} 2 \mathrm{~N}) 2 \mathrm{P}-\mathrm{DHAP}+\backslash \backslash 1,1 \backslash \mathrm{P}, 0.2131889426,1.2642694202,-0.8339966731 \backslash \mathrm{N}, 1$. $5227088205,0.5668990124,-1.5902669365 \backslash \mathrm{N},-1.1053243712,0.6349371154,-1$. $7509393519 \backslash \mathrm{C}, 2.8156203407,1.2409992059,-1.4725695205 \backslash \mathrm{C},-2.4228553869,1$ $.0113214486,-1.2194852826 \backslash \mathrm{C}, 1.6018487376,-0.788831531,-2.1205743167 \backslash \mathrm{C}$, $-1.0477959368,0.8846446722,-3.1986237832 \backslash \mathrm{H}, 2.6812718981,2.2515540008,-$ $1.0771102892 \backslash \mathrm{H},-2.4824432252,0.822313692,-0.144470809 \backslash \mathrm{H}, 0.6027424487,-$ $1.2142314133,-2.2286915926 \backslash \mathrm{H},-0.0729841191,0.6133950558,-3.6047712727 \backslash$ $\mathrm{H}, 3.4931468094,0.6877141495,-0.8091579745 \backslash \mathrm{H},-2.6660485292,2.0688957292$ $,-1.4004673248 \backslash \mathrm{H}, 3.2833894292,1.3193579173,-2.4606510317 \backslash \mathrm{H},-3.18612428$ $34,0.3978060959,-1.7093804278 \backslash \mathrm{H}, 2.0790427022,-0.775927262,-3.107712207$ $\backslash \mathrm{H},-1.8073796797,0.2636302941,-3.6849155106 \backslash \mathrm{H}, 2.1996325785,-1.43176637$ $79,-1.4603115922 \backslash \mathrm{H},-1.2524807656,1.9355614708,-3.4521083838 \backslash \mathrm{N},-0.13710$ $55386,0.1099776776,0.6659932886 \backslash \mathrm{C},-0.3675136783,-1.5072385336,2.938725$ $4309 \backslash \mathrm{C}, 0.2455017926,0.5696092231,1.8805819803 \backslash \mathrm{C},-0.6389136577,-1.14420$ $22071,0.5679517323 \backslash \mathrm{C}, 0.1477404952,-0.1892428789,3.0180078298 \backslash \mathrm{C},-0.7666$ $993551,-1.966359528,1.6573396002 \backslash \mathrm{N},-0.4772685566,-2.2838421964,4.02641$ $47084 \backslash \mathrm{H}, 0.6311507523,1.585085249,1.9020172384 \backslash \mathrm{H},-0.9531096128,-1.44060$ $74344,-0.4277352949 \backslash \mathrm{H}, 0.4618352246,0.2295933169,3.9686424983 \backslash \mathrm{H},-1.1786$ $143603,-2.962521844,1.5291136428 \backslash \mathrm{H},-0.2013947802,-1.9546820637,4.93972$ $75297 \backslash \mathrm{H},-0.8521392127,-3.2192123699,3.9713989144 \backslash \backslash$ Version=x86-Linux-G9 8RevA.11.1 \HF=-913.6970023 $\backslash \mathrm{RMSD}=3.407 e-09 \backslash \mathrm{RMSF}=9.187 \mathrm{e}-06 \backslash \mathrm{Dipole}=-0.063$ $4222,-1.2437973,1.7696301 \backslash \mathrm{PG}=\mathrm{C} 01 \quad[\mathrm{X}(\mathrm{C} 9 \mathrm{H} 18 \mathrm{~N} 4 \mathrm{P} 1)] \backslash \backslash \mathrm{Q}$

\section{Single Point Energy Calculation of $\left(\mathrm{Me}_{2} \mathrm{~N}\right)_{2} \mathrm{P}-\mathrm{DHAP}{ }^{+}, \mathbf{4}-\mathrm{NMe}_{2}$, in $C_{l}$ symmetry}

$1 \backslash 1 \backslash G I N C-N E I L \backslash S P \backslash R B 3 P W 91 \backslash 6-311+G(3 d f, 2 p) \backslash C 9 H 18 N 4 P 1(1+) \backslash B O B B Y \backslash 04-N o v-20$ $03 \backslash 0 \backslash \backslash \#$ B3PW91/6-311+G(3DF, 2P) SCF=TIGHT \#P GFINPUT IOP(6/7=3) TEST \S 
ingle Point and Molden of (Me2N)2P-DHPAP+\\1,1\P,0,-1.290482,-0.113563 $,-0.813104 \backslash \mathrm{N}, 0,-1.853652,-1.299297,0.211585 \backslash \mathrm{N}, 0,-1.712644,1.325123,0.0$ $39705 \backslash \mathrm{C}, 0,-2.172099,-2.604509,-0.367117 \backslash \mathrm{C}, 0,-1.22373,2.553362,-0.60246$ $9 \backslash \mathrm{C}, 0,-1.800139,-1.288686,1.668472 \backslash \mathrm{C}, 0,-3.134264,1.461854,0.388639 \backslash \mathrm{H}, 0$ $,-2.202822,-2.537789,-1.458109 \backslash \mathrm{H}, 0,-0.164616,2.468584,-0.859412 \backslash \mathrm{H}, 0,-1$ $.608003,-0.278931,2.035005 \backslash \mathrm{H}, 0,-3.510649,0.554776,0.862278 \backslash \mathrm{H}, 0,-1.4285$ $42,-3.358992,-0.078743 \backslash \mathrm{H}, 0,-1.783715,2.805697,-1.515215 \backslash \mathrm{H}, 0,-3.156647$, $-2.93453,-0.016396 \backslash \mathrm{H}, 0,-1.330886,3.383743,0.103196 \backslash \mathrm{H}, 0,-2.75867,-1.627$ $356,2.079378 \backslash \mathrm{H}, 0,-3.235576,2.288187,1.099987 \backslash \mathrm{H}, 0,-1.013902,-1.962627,2$ $.034931 \backslash \mathrm{H}, 0,-3.762009,1.685982,-0.486734 \backslash \mathrm{N}, 0,0.576927,0.043898,-0.3737$ $22 \backslash \mathrm{C}, 0,3.317546,-0.016698,0.19119 \backslash \mathrm{C}, 0,1.449398,-0.506,-1.250809 \backslash \mathrm{C}, 0,1$. $051601,0.569977,0.780538 \backslash \mathrm{C}, 0,2.798602,-0.554342,-1.013186 \backslash \mathrm{C}, 0,2.386202$ $, 0.558699,1.093192 \backslash \mathrm{N}, 0,4.630153,-0.046175,0.463992 \backslash \mathrm{H}, 0,1.014923,-0.903$ $615,-2.163776 \backslash \mathrm{H}, 0,0.304153,1.020305,1.425901 \backslash \mathrm{H}, 0,3.455986,-0.999971,-1$ $.752811 \backslash \mathrm{H}, 0,2.719098,0.996613,2.02907 \backslash \mathrm{H}, 0,5.294235,-0.447748,-0.181242$ $\backslash \mathrm{H}, 0,5.000678,0.34433,1.317625 \backslash \backslash$ Version=x86-Linux-G98RevA. $11.1 \backslash \mathrm{HF}=-913$ $.9224432 \backslash \mathrm{RMSD}=4.302 e-09 \backslash \mathrm{Dipole}=2.06105,-0.1413257,0.4397329 \backslash \mathrm{PG}=\mathrm{C} 01 \quad[\mathrm{X}($ C9H18N4P1) ] \\@

\section{Single Point Energy Calculation of the $\left(\mathrm{Me}_{2} \mathrm{~N}\right)_{2} \mathrm{P}+$ Fragment from $\left(\mathrm{Me}_{2} \mathrm{~N}\right)_{2} \mathrm{P}-\mathrm{DHAP}{ }^{+}$, 4- $\mathbf{N M e}_{2}$, in $C_{1}$ symmetry}

$1 \backslash 1 \backslash G I N C-N E I L \backslash S P \backslash R B 3 P W 91 \backslash 6-311+G(3 d f, 2 p) \backslash C 4 H 12 N 2 P 1(1+) \backslash B O B B Y \backslash 04-N o v-20$ $03 \backslash 0 \backslash \backslash \#$ B3PW91/6-311+G(3DF, 2P) SCF=TIGHT \#P GFINPUT IOP (6/7=3) TEST \\S ingle Point and Molden of (Me2N)2P-DHPAP+\\1,1\P,0,-1.290482,-0.113563 $,-0.813104 \backslash \mathrm{N}, 0,-1.853652,-1.299297,0.211585 \backslash \mathrm{N}, 0,-1.712644,1.325123,0.0$ $39705 \backslash \mathrm{C}, 0,-2.172099,-2.604509,-0.367117 \backslash \mathrm{C}, 0,-1.22373,2.553362,-0.60246$ $9 \backslash \mathrm{C}, 0,-1.800139,-1.288686,1.668472 \backslash \mathrm{C}, 0,-3.134264,1.461854,0.388639 \backslash \mathrm{H}, 0$ $,-2.202822,-2.537789,-1.458109 \backslash \mathrm{H}, 0,-0.164616,2.468584,-0.859412 \backslash \mathrm{H}, 0,-1$ $.608003,-0.278931,2.035005 \backslash \mathrm{H}, 0,-3.510649,0.554776,0.862278 \backslash \mathrm{H}, 0,-1.4285$ $42,-3.358992,-0.078743 \backslash \mathrm{H}, 0,-1.783715,2.805697,-1.515215 \backslash \mathrm{H}, 0,-3.156647$, $-2.93453,-0.016396 \backslash \mathrm{H}, 0,-1.330886,3.383743,0.103196 \backslash \mathrm{H}, 0,-2.75867,-1.627$ $356,2.079378 \backslash \mathrm{H}, 0,-3.235576,2.288187,1.099987 \backslash \mathrm{H}, 0,-1.013902,-1.962627,2$ $.034931 \backslash \mathrm{H}, 0,-3.762009,1.685982,-0.486734 \backslash \backslash$ Version=x86-Linux-G98RevA. 11 $.1 \backslash \mathrm{HF}=-610.2205916 \backslash \mathrm{RMSD}=4.563 e-09 \backslash \mathrm{Dipole}=-0.1531409,-0.3060682,0.02452$ $23 \backslash \mathrm{PG}=\mathrm{C} 01 \quad[\mathrm{X}(\mathrm{C} 4 \mathrm{H} 12 \mathrm{~N} 2 \mathrm{P} 1)] \backslash \backslash \mathrm{C}$

\section{Single Point Energy Calculation of the DHAP Fragment from $\left(\mathrm{Me}_{2} \mathrm{~N}\right)_{2} \mathrm{P}-\mathrm{DHAP}^{+}, 4-$ $\mathbf{N M e}_{2}$, in $C_{1}$ symmetry}

$1 \backslash 1 \backslash G I N C-N E I L \backslash S P \backslash R B 3 P W 91 \backslash 6-311+G(3 d f, 2 p) \backslash C 5 H 6 N 2 \backslash B O B B Y \backslash 05-N o v-2003 \backslash 0 \backslash \backslash \#$ B3PW91/6-311+G(3DF, 2P) SCF=TIGHT \#P GFINPUT IOP(6/7=3) TEST \\Single P

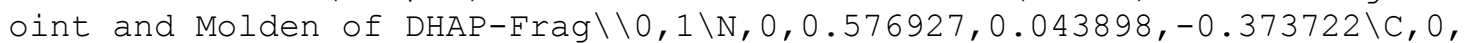
$3.317546,-0.016698,0.19119 \backslash \mathrm{C}, 0,1.449398,-0.506,-1.250809 \backslash \mathrm{C}, 0,1.051601$, $0.569977,0.780538 \backslash \mathrm{C}, 0,2.798602,-0.554342,-1.013186 \backslash \mathrm{C}, 0,2.386202,0.5586$ $99,1.093192 \backslash \mathrm{N}, 0,4.630153,-0.046175,0.463992 \backslash \mathrm{H}, 0,1.014923,-0.903615,-2$. $163776 \backslash \mathrm{H}, 0,0.304153,1.020305,1.425901 \backslash \mathrm{H}, 0,3.455986,-0.999971,-1.752811$ $\backslash \mathrm{H}, 0,2.719098,0.996613,2.02907 \backslash \mathrm{H}, 0,5.294235,-0.447748,-0.181242 \backslash \mathrm{H}, 0,5$. $000678,0.34433,1.317625 \backslash \backslash$ Version=x86-Linux-G98RevA.11.1 \HF=-303.627756 $1 \backslash \mathrm{RMSD}=3.018 \mathrm{e}-09 \backslash \mathrm{Dipole}=1.7437603,-0.0215119,0.3514957 \backslash \mathrm{PG}=\mathrm{C} 01 \quad[\mathrm{X}(\mathrm{C} 5 \mathrm{H} 6 \mathrm{~N}$ 2) $] \backslash \backslash @$ 


\section{Geometry Optimization of $\mathrm{Ph}_{2} \mathrm{P}-$ Carbene ${ }^{+}, \mathbf{5 - P h}$, in $C_{1}$ symmetry}

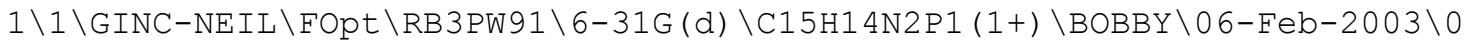
$\backslash \backslash \#$ B3PW91/6-31G(D) OPT FREQ=NORAMAN POP=(FULL, NBO) TEST \\Geom and Fre $\mathrm{q}$ of $\mathrm{Ph} 2 \mathrm{P}-\mathrm{CarbH}+\backslash \backslash 1,1 \backslash \mathrm{P},-0.0209454117,0.8229173917,0.8336971257 \backslash \mathrm{C}, 1.57$ $02504148,0.6561719052,-0.0515857323 \backslash C,-1.3740193806,0.7860000655,-0.41$ $01354177 \backslash C, 1.9741456357,-0.4835421719,-0.7678588867 \backslash C,-2.657735847,1.1$ $128352208,0.0608295645 \backslash \mathrm{C}, 2.4264748187,1.7661166056,0.0136226612 \backslash \mathrm{C},-1.1$ $898371686,0.4999917003,-1.7692047227 \backslash C, 3.2102543534,-0.5059009616,-1.4$ $072582971 \backslash \mathrm{C},-3.7461546992,1.1119164542,-0.810733825 \backslash \mathrm{C}, 3.6583010979,1.7$ $418353777,-0.6376022315 \backslash \mathrm{C},-2.2789729678,0.5222931246,-2.6379535458 \backslash \mathrm{C}, 4$ $.0506291778,0.6072058893,-1.3448511649 \backslash C,-3.555980341,0.8176321823,-2$. $1603452433 \backslash \mathrm{H}, 1.3242822948,-1.3533858568,-0.8325477758 \backslash \mathrm{H},-2.802452316,1$ $.3965622679,1.1020962152 \backslash \mathrm{H}, 2.1295756681,2.6488056333,0.5750755978 \backslash \mathrm{H},-0$ $.1983985182,0.2793533735,-2.1538280651 \backslash \mathrm{H}, 3.5171533054,-1.3900749091,-1$ $.9591056842 \backslash \mathrm{H},-4.7350515441,1.3663303098,-0.4398164812 \backslash \mathrm{H}, 4.3125636518$, $2.6072234667,-0.5859633846 \backslash \mathrm{H},-2.1283559249,0.3105555118,-3.6928327815 \backslash$ $\mathrm{H}, 5.0136891674,0.5867797027,-1.8470667193 \backslash \mathrm{H},-4.4004103676,0.8342682876$ $,-2.8435019705 \backslash \mathrm{C},-0.2122549071,-0.9019478687,1.4575702408 \backslash \mathrm{N}, 0.64948674$ $76,-1.5882192723,2.229595294 \backslash \mathrm{N},-1.3192822421,-1.6597559774,1.404101394$ $5 \backslash C, 0.0926132909,-2.7769023258,2.6547298152 \backslash C,-1.1620015097,-2.8203032$ $567,2.1326334395 \backslash \mathrm{H}, 1.5866770377,-1.2685612002,2.4419319884 \backslash \mathrm{H},-2.140106$ $0845,-1.3831832668,0.8740743794 \backslash \mathrm{H}, 0.6275036812,-3.4774623737,3.2772149$ $581 \backslash \mathrm{H},-1.9381922241,-3.5655567191,2.2117960937 \backslash \backslash$ Version=x86-Linux-G98R evA.11. $1 \backslash \mathrm{HF}=-1030.3606223 \backslash \mathrm{RMSD}=9.009 \mathrm{e}-09 \backslash \mathrm{RMSF}=9.624 \mathrm{e}-06 \backslash \mathrm{Dipole}=-0.1713$ $983,-2.0720519,1.3729107 \backslash \mathrm{PG}=\mathrm{C} 01 \quad[\mathrm{X}(\mathrm{C} 15 \mathrm{H} 14 \mathrm{~N} 2 \mathrm{P} 1)] \backslash \backslash @$

\section{Single Point Energy Calculation of $\mathrm{Ph}_{2} \mathrm{P}-$ Carbene ${ }^{+}, \mathbf{5}-\mathrm{Ph}$, in $C_{1}$ symmetry}

$1 \backslash 1 \backslash G I N C-N E I L \backslash S P \backslash R B 3 P W 91 \backslash 6-311+G(3 d f, 2 p) \backslash C 15 H 14 N 2 P 1(1+) \backslash B O B B Y \backslash 09-F e b-2$ $003 \backslash 0 \backslash \backslash \#$ B3PW91/6-311+G(3DF, 2P) SCF=TIGHT \#P GFINPUT IOP $(6 / 7=3)$ TEST \ Single Point and Molden of Ph2P-CarbH+\\1,1\P,0,0.030107,0.097797,-1.1 $67138 \backslash \mathrm{C}, 0,1.596589,-0.441609,-0.39344 \backslash \mathrm{C}, 0,-1.344896,-0.843854,-0.39128$ $3 \backslash \mathrm{C}, 0,1.937047,-0.238299,0.954848 \backslash \mathrm{C}, 0,-2.604437,-0.718494,-1.003163 \backslash \mathrm{C}$, $0,2.50208,-1.10061,-1.239107 \backslash \mathrm{C}, 0,-1.197423,-1.687309,0.717603 \backslash \mathrm{C}, 0,3.15$ $9546,-0.690319,1.443183 \backslash \mathrm{C}, 0,-3.706959,-1.396708,-0.484752 \backslash \mathrm{C}, 0,3.720009$ $,-1.560428,-0.741818 \backslash \mathrm{C}, 0,-2.299585,-2.378496,1.216542 \backslash \mathrm{C}, 0,4.049296,-1$. $353749,0.596226 \backslash \mathrm{C}, 0,-3.553701,-2.227505,0.624531 \backslash \mathrm{H}, 0,1.248312,0.268156$ $, 1.627367 \backslash \mathrm{H}, 0,-2.718167,-0.114332,-1.901904 \backslash \mathrm{H}, 0,2.254412,-1.252726,-2$. $286981 \backslash \mathrm{H}, 0,-0.223616,-1.82066,1.179647 \backslash \mathrm{H}, 0,3.417305,-0.529787,2.486387$ $\backslash \mathrm{H}, 0,-4.676974,-1.295691,-0.963385 \backslash \mathrm{H}, 0,4.412712,-2.073829,-1.402275 \backslash \mathrm{H}$, $0,-2.177016,-3.039718,2.069801 \backslash \mathrm{H}, 0,5.001548,-1.707046,0.981607 \backslash \mathrm{H}, 0,-4$. $408428,-2.770261,1.018105 \backslash \mathrm{C}, 0,-0.226328,1.690324,-0.273227 \backslash \mathrm{N}, 0,0.61763$ $2,2.736287,-0.215941 \backslash \mathrm{N}, 0,-1.366557,2.125385,0.286452 \backslash \mathrm{C}, 0,0.016275,3.82$ $3554,0.384088 \backslash \mathrm{C}, 0,-1.248,3.435835,0.700012 \backslash \mathrm{H}, 0,1.571626,2.704184,-0.55$ $426 \backslash \mathrm{H}, 0,-2.18352,1.530276,0.384381 \backslash \mathrm{H}, 0,0.530498,4.760176,0.534733 \backslash \mathrm{H}, 0$, $-2.054885,3.968584,1.17882 \backslash \backslash$ Version=x86-Linux-G98RevA $.11 .1 \backslash H F=-1030.60$ $15074 \backslash \mathrm{RMSD}=5.432 e-09 \backslash \mathrm{Dipole}=-0.236323,2.2700158,0.607429 \backslash \mathrm{PG}=\mathrm{C} 01] \mathrm{X}(\mathrm{C} 15$ $\mathrm{H} 14 \mathrm{~N} 2 \mathrm{P} 1)] \backslash \backslash \mathrm{Q}$ 


\section{Single Point Energy Calculation of the $\mathrm{Ph}_{2} \mathrm{P}^{+}$Fragment from $\mathrm{Ph}_{2} \mathrm{P}-$ Carbene ${ }^{+}$, 5-Ph, in $C_{l}$ symmetry}

$1 \backslash 1 \backslash G I N C-N E I L \backslash S P \backslash R B 3 P W 91 \backslash 6-311+G(3 d f, 2 p) \backslash C 12 H 10 P 1(1+) \backslash B O B B Y \backslash 09-F e b-200$ $3 \backslash 0 \backslash \backslash \#$ B3PW91/6-311+G(3DF,2P) SCF=TIGHT \#P GFINPUT IOP (6/7=3) TEST \\i

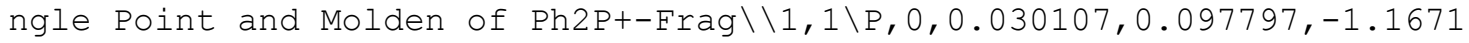
$38 \backslash \mathrm{C}, 0,1.596589,-0.441609,-0.39344 \backslash \mathrm{C}, 0,-1.344896,-0.843854,-0.391283 \backslash \mathrm{C}$ $, 0,1.937047,-0.238299,0.954848 \backslash \mathrm{C}, 0,-2.604437,-0.718494,-1.003163 \backslash \mathrm{C}, 0,2$ $.50208,-1.10061,-1.239107 \backslash \mathrm{C}, 0,-1.197423,-1.687309,0.717603 \backslash \mathrm{C}, 0,3.15954$ $6,-0.690319,1.443183 \backslash \mathrm{C}, 0,-3.706959,-1.396708,-0.484752 \backslash \mathrm{C}, 0,3.720009,-1$ $.560428,-0.741818 \backslash \mathrm{C}, 0,-2.299585,-2.378496,1.216542 \backslash \mathrm{C}, 0,4.049296,-1.353$ $749,0.596226 \backslash \mathrm{C}, 0,-3.553701,-2.227505,0.624531 \backslash \mathrm{H}, 0,1.248312,0.268156,1$. $627367 \backslash \mathrm{H}, 0,-2.718167,-0.114332,-1.901904 \backslash \mathrm{H}, 0,2.254412,-1.252726,-2.286$ $981 \backslash \mathrm{H}, 0,-0.223616,-1.82066,1.179647 \backslash \mathrm{H}, 0,3.417305,-0.529787,2.486387 \backslash \mathrm{H}$, $0,-4.676974,-1.295691,-0.963385 \backslash \mathrm{H}, 0,4.412712,-2.073829,-1.402275 \backslash \mathrm{H}, 0,-$ $2.177016,-3.039718,2.069801 \backslash \mathrm{H}, 0,5.001548,-1.707046,0.981607 \backslash \mathrm{H}, 0,-4.408$ $428,-2.770261,1.018105 \backslash \backslash V e r s i o n=x 86-L i n u x-G 98 R e v A .11 .1 \backslash H F=-804.2939296$ $\backslash \mathrm{RMSD}=7.644 \mathrm{e}-09 \backslash \mathrm{Dipole}=-0.7068848,-0.2588541,0.1398106 \backslash \mathrm{PG}=\mathrm{C} 01 \quad[\mathrm{X}(\mathrm{C} 12 \mathrm{H} 1$ $0 \mathrm{P} 1)] \backslash \backslash @$

\section{Single Point Energy Calculation of the Carbene Fragment from $\mathrm{Ph}_{2} \mathrm{P}-$ Carbene ${ }^{+}, \mathbf{5 - P h}$, in $C_{l}$ symmetry}

$1 \backslash 1 \backslash G I N C-N E I L \backslash S P \backslash R B 3 P W 91 \backslash 6-311+G(3 d f, 2 p) \backslash C 3 H 4 N 2 \backslash B O B B Y \backslash 09-F e b-2003 \backslash 0 \backslash \backslash \#$ B3PW91/6-311+G(3DF,2P) SCF=TIGHT \#P GFINPUT IOP(6/7=3) TEST \Single P

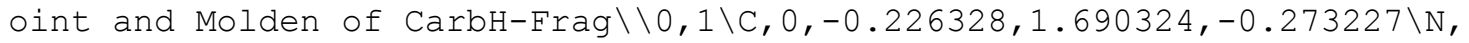
$0,0.617632,2.736287,-0.215941 \backslash \mathrm{N}, 0,-1.366557,2.125385,0.286452 \backslash \mathrm{C}, 0,0.01$ $6275,3.823554,0.384088 \backslash \mathrm{C}, 0,-1.248,3.435835,0.700012 \backslash \mathrm{H}, 0,1.571626,2.704$ $184,-0.55426 \backslash \mathrm{H}, 0,-2.18352,1.530276,0.384381 \backslash \mathrm{H}, 0,0.530498,4.760176,0.53$ $4733 \backslash \mathrm{H}, 0,-2.054885,3.968584,1.17882 \backslash \backslash$ Version=x86-Linux-G98RevA.11.1 $\backslash \mathrm{HF}$ $=-226.1647446 \backslash \mathrm{RMSD}=3.645 e-09 \backslash \mathrm{Dipole}=-0.2026812,1.0301197,0.4486864 \backslash \mathrm{PG}=$ $\mathrm{C} 01[\mathrm{X}(\mathrm{C} 3 \mathrm{H} 4 \mathrm{~N} 2)] \backslash \backslash \mathrm{Q}$

\section{Geometry Optimization of $\left(\mathrm{Me}_{2} \mathrm{~N}\right)_{2} \mathrm{P}-\mathrm{Carbene}^{+}, \mathbf{5}-\mathrm{NMe}_{2}$, in $C_{s}$ symmetry}

$1 \backslash 1 \backslash G I N C-N E I L \backslash F O p t \backslash R B 3 P W 91 \backslash 6-31 G(d) \backslash C 7 H 16 N 4 P 1(1+) \backslash B O B B Y \backslash 11-$ Feb-2003\0 \\# B3PW91/6-31G(D) OPT FREQ=NORAMAN POP=(FULL, NBO) TEST \Geom and Freq of $(\mathrm{Me} 2 \mathrm{~N}) 2 \mathrm{P}-\mathrm{CarbH}+-\mathrm{Cs}-\mathrm{side} \backslash \backslash 1,1 \backslash \mathrm{P}, 0.7897785571,0 .,-0.6344955716 \backslash \mathrm{C}, 0.2$ $107909317,0 ., 1.1168855178 \backslash \mathrm{N}, 0.0319734267,-1.4609246123,-1.0809438339 \backslash \mathrm{N}$ $, 0.0319734267,1.4609246123,-1.0809438339 \backslash \mathrm{C}, 0.725229411,-2.2372978853,-$ $2.1173891017 \backslash \mathrm{C}, 0.725229411,2.2372978853,-2.1173891017 \backslash \mathrm{C},-1.410849714,-$ $1.7009049723,-1.0709371269 \backslash \mathrm{C},-1.410849714,1.7009049723,-1.0709371269 \backslash \mathrm{H}$ $, 1.7991251616,-2.0366923894,-2.0807703339 \backslash \mathrm{H}, 1.7991251616,2.0366923894$, $-2.0807703339 \backslash \mathrm{H},-1.5987059655,-2.7768583113,-0.973990506 \backslash \mathrm{H},-1.59870596$ $55,2.7768583113,-0.973990506 \backslash \mathrm{H}, 0.5678953202,-3.306357001,-1.9351520137$ $\backslash \mathrm{H}, 0.3562290344,2.0024457494,-3.1248511988 \backslash \mathrm{H}, 0.3562290344,-2.002445749$ $4,-3.1248511988 \backslash \mathrm{H}, 0.5678953202,3.306357001,-1.9351520137 \backslash \mathrm{H},-1.88545780$ $9,-1.1960009123,-0.2249197813 \backslash \mathrm{H},-1.8951685039,1.3598108048,-1.99589738$ $78 \backslash \mathrm{H},-1.8951685039,-1.3598108048,-1.9958973878 \backslash \mathrm{H},-1.885457809,1.196000$ $9123,-0.2249197813 \backslash \mathrm{N}, 0.0947964278,1.0677218934,1.9218290865 \backslash \mathrm{N}, 0.094796$ $4278,-1.0677218934,1.9218290865 \backslash \mathrm{C},-0.1010785928,0.6808145545,3.2302130$ $749 \backslash \mathrm{C},-0.1010785928,-0.6808145545,3.2302130749 \backslash \mathrm{H}, 0.1583154105,2.016741$ 
$2337,1.571325536 \backslash \mathrm{H}, 0.1583154105,-2.0167412337,1.571325536 \backslash \mathrm{H},-0.2251402$ $281,1.3891767319,4.0347980729 \backslash \mathrm{H},-0.2251402281,-1.3891767319,4.03479807$ $29 \backslash \backslash$ Version=x86-Linux-G98RevA.11. $1 \backslash$ State $=1-A^{\prime} \backslash \mathrm{HF}=-836.2899198 \backslash \mathrm{RMSD}=3.6$ $80 e-09 \backslash \mathrm{RMSF}=7.006 e-06 \backslash \mathrm{Dipole}=-0.501233,0 ., 1.6312818 \backslash \mathrm{PG}=\mathrm{CS} \quad[\mathrm{SG}(\mathrm{C} 1 \mathrm{P} 1), \mathrm{X}($ $\mathrm{C} 6 \mathrm{H} 16 \mathrm{~N} 4)] \backslash \backslash @$

\section{Single Point Energy Calculation of $\left(\mathrm{Me}_{2} \mathrm{~N}\right)_{2} \mathrm{P}-$ Carbene ${ }^{+}, \mathbf{5}-\mathrm{NMe}_{2}$, in $C_{s}$ symmetry}

$1 \backslash 1 \backslash G I N C-N E I L \backslash S P \backslash R B 3 P W 91 \backslash 6-311+G(3 \mathrm{df}, 2 \mathrm{p}) \backslash \mathrm{C} 7 \mathrm{H} 16 \mathrm{~N} 4 \mathrm{P} 1(1+) \backslash \mathrm{BOBBY} \backslash 12-\mathrm{Feb}-20$ $03 \backslash 0 \backslash \backslash \#$ B3PW91/6-311+G(3DF, 2P) SCF=TIGHT \#P GFINPUT IOP $(6 / 7=3)$ TEST \\S

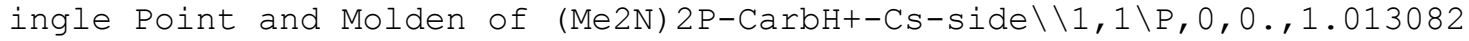
$, 0 . \backslash \mathrm{C}, 0,-1.002721,-0.53518,0 . \backslash \mathrm{N}, 0,0.822657,0.701924,1.460925 \backslash \mathrm{N}, 0,0.822$ $657,0.701924,-1.460925 \backslash \mathrm{C}, 0,1.196462,1.8915,2.237298 \backslash \mathrm{C}, 0,1.196462,1.891$ $5,-2.237298 \backslash C, 0,1.7185,-0.42914,1.700905 \backslash C, 0,1.7185,-0.42914,-1.700905$ $\backslash \mathrm{H}, 0,0.495331,2.705754,2.036692 \backslash \mathrm{H}, 0,0.495331,2.705754,-2.036692 \backslash \mathrm{H}, 0,1$. $760577,-0.636307,2.776858 \backslash \mathrm{H}, 0,1.760577,-0.636307,-2.776858 \backslash \mathrm{H}, 0,1.15293$ $2,1.65471,3.306357 \backslash \mathrm{H}, 0,2.212965,2.234811,-2.002446 \backslash \mathrm{H}, 0,2.212965,2.2348$ $11,2.002446 \backslash \mathrm{H}, 0,1.152932,1.65471,-3.306357 \backslash \mathrm{H}, 0,1.35621,-1.328998,1.196$ $001 \backslash \mathrm{H}, 0,2.742911,-0.227401,-1.359811 \backslash \mathrm{H}, 0,2.742911,-0.227401,1.359811 \backslash \mathrm{H}$ $, 0,1.35621,-1.328998,-1.196001 \backslash \mathrm{N}, 0,-1.557591,-1.129745,-1.067722 \backslash \mathrm{N}, 0,-$ $1.557591,-1.129745,1.067722 \backslash C, 0,-2.454904,-2.101889,-0.680815 \backslash C, 0,-2.4$ $54904,-2.101889,0.680815 \backslash \mathrm{H}, 0,-1.324128,-0.860705,-2.016741 \backslash \mathrm{H}, 0,-1.3241$ $28,-0.860705,2.016741 \backslash \mathrm{H}, 0,-3.004443,-2.702519,-1.389177 \backslash \mathrm{H}, 0,-3.004443$, $-2.702519,1.389177 \backslash \backslash$ Version=x86-Linux-G98RevA.11.1 $\backslash$ State $=1-A \cdot \backslash H F=-836$. $4905427 \backslash \mathrm{RMS} D=3.519 \mathrm{e}-09 \backslash \mathrm{Dipole}=-0.9177906,-1.3590076,0 . \backslash \mathrm{PG}=\mathrm{CS} \quad[\mathrm{SG}(\mathrm{C} 1 \mathrm{P} 1)$ $, \mathrm{X}(\mathrm{C} 6 \mathrm{H} 16 \mathrm{~N} 4)] \backslash \backslash @$

\section{Single Point Energy Calculation of the $\left(\mathrm{Me}_{2} \mathrm{~N}\right)_{2} \mathrm{P}^{+}$Fragment from $\left(\mathrm{Me}_{2} \mathrm{~N}\right)_{2} \mathrm{P}-$ Carbene ${ }^{+}, 5-$ $\mathbf{N M e}_{2}$, in $C_{s}$ symmetry}

$1 \backslash 1 \backslash G I N C-N E I L \backslash S P \backslash R B 3 P W 91 \backslash 6-311+G(3 \mathrm{df}, 2 \mathrm{p}) \backslash \mathrm{C} 4 \mathrm{H} 12 \mathrm{~N} 2 \mathrm{P} 1(1+) \backslash \mathrm{BOBBY} \backslash 12-\mathrm{Feb}-20$ $03 \backslash 0 \backslash \backslash \#$ B3PW91/6-311+G(3DF, 2P) SCF=TIGHT \#P GFINPUT IOP $(6 / 7=3) \quad$ TEST $\backslash \backslash S$ ingle Point and Molden of (Me2N) 2P+-Cs-side-Frag \\1,1\P,0,0.,1.013082, $0 . \backslash \mathrm{N}, 0,0.822657,0.701924,1.460925 \backslash \mathrm{N}, 0,0.822657,0.701924,-1.460925 \backslash \mathrm{C}, 0$, $1.196462,1.8915,2.237298 \backslash \mathrm{C}, 0,1.196462,1.8915,-2.237298 \backslash \mathrm{C}, 0,1.7185,-0.4$ $2914,1.700905 \backslash \mathrm{C}, 0,1.7185,-0.42914,-1.700905 \backslash \mathrm{H}, 0,0.495331,2.705754,2.03$ $6692 \backslash \mathrm{H}, 0,0.495331,2.705754,-2.036692 \backslash \mathrm{H}, 0,1.760577,-0.636307,2.776858 \backslash \mathrm{H}$ $, 0,1.760577,-0.636307,-2.776858 \backslash \mathrm{H}, 0,1.152932,1.65471,3.306357 \backslash \mathrm{H}, 0,2.21$ $2965,2.234811,-2.002446 \backslash \mathrm{H}, 0,2.212965,2.234811,2.002446 \backslash \mathrm{H}, 0,1.152932,1$. $65471,-3.306357 \backslash \mathrm{H}, 0,1.35621,-1.328998,1.196001 \backslash \mathrm{H}, 0,2.742911,-0.227401$, $-1.359811 \backslash \mathrm{H}, 0,2.742911,-0.227401,1.359811 \backslash \mathrm{H}, 0,1.35621,-1.328998,-1.196$ $001 \backslash \backslash$ Version=x86-Linux-G98RevA.11.1 \State $=1-A^{\prime} \backslash \mathrm{HF}=-610.1832757 \backslash \mathrm{RMSD}=8$. $509 e-09 \backslash$ Dipole $=0.5100684,-0.001356,0 . \backslash P G=C S \quad[S G(P 1), X(C 4 H 12 N 2)] \backslash \backslash @$

\section{Single Point Energy Calculation of the Carbene Fragment from $\left(\mathrm{Me}_{2} \mathrm{~N}\right)_{2} \mathrm{P}-$ Carbene ${ }^{+}, 5-$ $\mathbf{N M e}_{2}$, in $C_{s}$ symmetry}

$1 \backslash 1 \backslash G I N C-N E I L \backslash S P \backslash R B 3 P W 91 \backslash 6-311+G(3 d f, 2 p) \backslash C 3 H 4 N 2 \backslash B O B B Y \backslash 12-F e b-2003 \backslash 0 \backslash \backslash \#$ B3PW91/6-311+G(3DF, 2P) SCF=TIGHT \#P GFINPUT IOP(6/7=3) TEST \\ingle P

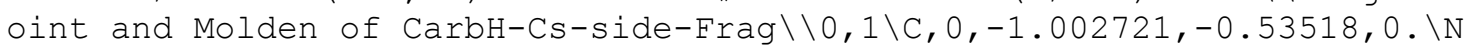
$, 0,-1.557591,-1.129745,-1.067722 \backslash \mathrm{N}, 0,-1.557591,-1.129745,1.067722 \backslash \mathrm{C}, 0$, 
$-2.454904,-2.101889,-0.680815 \backslash \mathrm{C}, 0,-2.454904,-2.101889,0.680815 \backslash \mathrm{H}, 0,-1$. $324128,-0.860705,-2.016741 \backslash \mathrm{H}, 0,-1.324128,-0.860705,2.016741 \backslash \mathrm{H}, 0,-3.004$ $443,-2.702519,-1.389177 \backslash H, 0,-3.004443,-2.702519,1.389177 \backslash \backslash$ Version $=x 86-$ Linux-G98RevA.11.1 \State=1-A' $\backslash \mathrm{HF}=-226.1645331 \backslash \mathrm{RMSD}=4.583 \mathrm{e}-09 \backslash \mathrm{Dipole}=-0$ $.7759354,-0.824174,0 . \backslash \mathrm{PG}=\mathrm{CS} \quad[\mathrm{SG}(\mathrm{C} 1), \mathrm{X}(\mathrm{C} 2 \mathrm{H} 4 \mathrm{~N} 2)] \backslash \backslash \mathrm{Q}$

\section{STRUCTURAL ALTERNATIVE CALCULATIONS (Schemes 4 and 5)}

\section{Geometry Optimization of Planar Carbene-P(NMe $)_{2}{ }^{+}$in $C_{2}$ symmetry}

$1 \backslash 1 \backslash G I N C-N E I L \backslash F O p t \backslash R B 3 P W 91 \backslash 6-31 G(d) \backslash C 7 H 16 N 4 P 1(1+) \backslash B O B B Y \backslash 30-O c t-2003 \backslash 0 \backslash$ $\backslash \#$ B3PW91/6-31G(D) OPT FREQ=NORAMAN POP=(FULL, NBO) TEST \\Geom and Freq

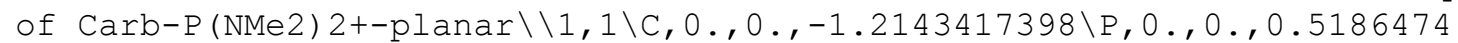
$929 \backslash \mathrm{N}, 1.0511920715,0.2382412741,-2.0330687275 \backslash \mathrm{N},-1.0511920715,-0.23824$ $12741,-2.0330687275 \backslash \mathrm{C}, 0.6593262605,0.1493687119,-3.3623033078 \backslash \mathrm{C},-0.659$ $3262605,-0.1493687119,-3.3623033078 \backslash \mathrm{H}, 1.9797168588,0.4485188969,-1.695$ $5231303 \backslash \mathrm{H},-1.9797168588,-0.4485188969,-1.6955231303 \backslash \mathrm{H}, 1.3515292065,0.3$ $065843089,-4.1743275831 \backslash \mathrm{H},-1.3515292065,-0.3065843089,-4.1743275831 \backslash \mathrm{N}$, $1.5029215232,0.3402267006,1.1472244269 \backslash \mathrm{N},-1.5029215232,-0.3402267006,1$ $.1472244269 \backslash \mathrm{C}, 2.3265858338,-0.732036549,1.7298787542 \backslash \mathrm{C},-2.3265858338,0$ $.732036549,1.7298787542 \backslash \mathrm{C}, 1.7844630351,1.6628362015,1.7298039616 \backslash \mathrm{C},-1$. $7844630351,-1.6628362015,1.7298039616 \backslash \mathrm{H}, 3.3828837001,-0.4596006822,1.6$ $2638664 \backslash \mathrm{H},-3.3828837001,0.4596006822,1.62638664 \backslash \mathrm{H}, 2.1132341415,-0.8779$ $972892,2.7962271925 \backslash \mathrm{H},-2.1132341415,0.8779972892,2.7962271925 \backslash \mathrm{H}, 2.1501$ $272893,-1.6684242684,1.1974051173 \backslash \mathrm{H},-2.1501272893,1.6684242684,1.19740$ $51173 \backslash \mathrm{H}, 1.2214569351,2.4317204071,1.1975476932 \backslash \mathrm{H},-1.2214569351,-2.4317$ $204071,1.1975476932 \backslash \mathrm{H}, 1.5294918082,1.7025723648,2.796261 \backslash \mathrm{H},-1.52949180$ $82,-1.7025723648,2.796261 \backslash \mathrm{H}, 2.8550157749,1.8720704173,1.625825749 \backslash \mathrm{H},-2$ $.8550157749,-1.8720704173,1.625825749 \backslash \backslash$ Version=x86-Linux-G98RevA.11.1\} State $=1-\mathrm{A} \backslash \mathrm{HF}=-836.2375588 \backslash \mathrm{RMSD}=3.388 \mathrm{e}-09 \backslash \mathrm{RMSF}=1.384 \mathrm{e}-05 \backslash \mathrm{Dipole}=0 ., 0 .,-$ $0.8198459 \backslash \mathrm{PG}=\mathrm{C} 02 \quad[\mathrm{C} 2(\mathrm{C} 1 \mathrm{P} 1), \mathrm{X}(\mathrm{C} 6 \mathrm{H} 16 \mathrm{~N} 4)] \backslash \backslash @$

\section{Single Point Energy Calculation of Planar Carbene-P(NMe $)_{2}{ }_{2}^{+}$in $C_{2}$ symmetry}

$1 \backslash 1 \backslash G I N C-N E I L \backslash S P \backslash R B 3 P W 91 \backslash 6-311+G(3 d f, 2 p) \backslash C 7 H 16 N 4 P 1(1+) \backslash B O B B Y \backslash 14-N o v-20$ $03 \backslash 0 \backslash \backslash \#$ B3PW91/6-311+G(3DF, 2P) SCF=TIGHT \#P GFINPUT IOP $(6 / 7=3) \quad \mathrm{TEST} \backslash \backslash \mathrm{S}$ ingle Point and Molden of Carb-P(NMe2)2-planar \\1,1\C,0,0.,0.,-1.21434 $2 \backslash \mathrm{P}, 0,0.0 .0 .518647 \backslash \mathrm{N}, 0,-0.000269,1.077851,-2.033069 \backslash \mathrm{N}, 0,0.000269,-1$. $077851,-2.033069 \backslash \mathrm{C}, 0,-0.00011,0.676034,-3.362303 \backslash \mathrm{C}, 0,0.00011,-0.676034$ $,-3.362303 \backslash \mathrm{H}, 0,-0.000348,2.029889,-1.695523 \backslash \mathrm{H}, 0,0.000348,-2.029889,-1$. $695523 \backslash \mathrm{H}, 0,-0.000614,1.385866,-4.174328 \backslash \mathrm{H}, 0,0.000614,-1.385866,-4.1743$ $28 \backslash \mathrm{N}, 0,0.1 .54095,1.147224 \backslash \mathrm{N}, 0,0 .,-1.54095,1.147224 \backslash \mathrm{C}, 0,1.227658,2.107$ $542,1.729879 \backslash \mathrm{C}, 0,-1.227658,-2.107542,1.729879 \backslash \mathrm{C}, 0,-1.227808,2.107563,1$ $.729804 \backslash \mathrm{C}, 0,1.227808,-2.107563,1.729804 \backslash \mathrm{H}, 0,1.195166,3.197923,1.626387$ $\backslash \mathrm{H}, 0,-1.195166,-3.197923,1.626387 \backslash \mathrm{H}, 0,1.322911,1.867229,2.796227 \backslash \mathrm{H}, 0,-$ $1.322911,-1.867229,2.796227 \backslash \mathrm{H}, 0,2.101977,1.728693,1.197405 \backslash \mathrm{H}, 0,-2.1019$ $77,-1.728693,1.197405 \backslash \mathrm{H}, 0,-2.102023,1.728213,1.197548 \backslash \mathrm{H}, 0,2.102023,-1$. $728213,1.197548 \backslash \mathrm{H}, 0,-1.322858,1.867657,2.796261 \backslash \mathrm{H}, 0,1.322858,-1.867657$

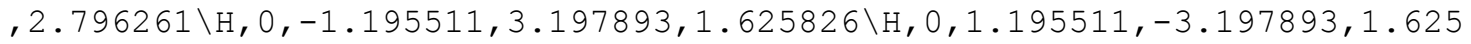
$826 \backslash \backslash$ Version=x86-Linux-G98RevA.11.1 \State=1-A \HF=-836.4400382 $\backslash$ RMSD=6.6 $20 e-09 \backslash$ Dipole=0.,0.,-0.6996718\PG=C02 [C2 (C1P1), X (C6H16N4) $\backslash \backslash @$ 


\section{Geometry Optimization of Planar $\mathrm{Me}_{3} \mathrm{P}-\mathrm{P}\left(\mathrm{NMe}_{2}\right)_{2}{ }^{+}$in $C_{s}$ symmetry}

$1 \backslash 1 \backslash G I N C-N E I L \backslash F O p t \backslash R B 3 P W 91 \backslash 6-31 G(d) \backslash C 7 H 21 N 2 P 2(1+) \backslash B O B B Y \backslash 12-N o v-2003 \backslash 0 \backslash$ $\backslash \#$ B3PW91/6-31G(D) OPT FREQ=NORAMAN POP=(FULL, NBO) TEST \\Geom and Freq

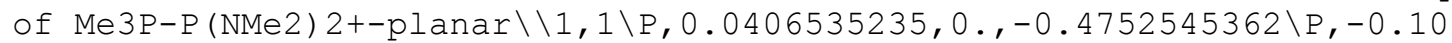
$15707973,0 ., 1.6051512465 \backslash \mathrm{C}, 1.5889483699,0.2 .2676331177 \backslash \mathrm{C},-0.973704293$ $2,1.4508015948,2.2854041108 \backslash \mathrm{C},-0.9737042932,-1.4508015948,2.2854041108$ $\backslash \mathrm{H}, 1.5374498472,0 ., 3.3614067762 \backslash \mathrm{H}, 2.1260235782,-0.8904418813,1.9326380$ $056 \backslash \mathrm{H}, 2.1260235782,0.8904418813,1.9326380056 \backslash \mathrm{H},-1.9770494528,1.5105108$ $683,1.85380712 \backslash \mathrm{H},-1.0603054836,1.3611057154,3.3739708247 \backslash \mathrm{H},-0.42384378$ $46,2.3612671904,2.0328935389 \backslash \mathrm{H},-1.9770494528,-1.5105108683,1.85380712 \backslash$ $\mathrm{H},-0.4238437846,-2.3612671904,2.0328935389 \backslash \mathrm{H},-1.0603054836,-1.36110571$ $54,3.3739708247 \backslash \mathrm{N},-1.4700273987,0 .,-1.1911854913 \backslash \mathrm{N}, 1.5730669007,0 .,-1$. $117120007 \backslash \mathrm{C},-2.0077801621,-1.2233503501,-1.8016140299 \backslash \mathrm{C},-2.0077801621$, $1.2233503501,-1.8016140299 \backslash \mathrm{C}, 2.1942909913,-1.2253384897,-1.6355781262 \backslash$ $\mathrm{C}, 2.1942909913,1.2253384897,-1.6355781262 \backslash \mathrm{H},-3.1017837798,-1.201088708$ $3,-1.7379903426 \backslash \mathrm{H},-1.7284094427,-1.30890274,-2.8599881681 \backslash \mathrm{H},-1.6397722$ $747,-2.1002787152,-1.2662495643 \backslash \mathrm{H}, 3.2672585362,-1.2090019689,-1.410399$ $2351 \backslash \mathrm{H}, 1.7475289947,-2.1013873636,-1.1629932337 \backslash \mathrm{H}, 2.0741851568,-1.3066$ $396958,-2.7237194973 \backslash \mathrm{H},-3.1017837798,1.2010887083,-1.7379903426 \backslash \mathrm{H},-1.6$ $397722747,2.1002787152,-1.2662495643 \backslash \mathrm{H},-1.7284094427,1.30890274,-2.859$ $9881681 \backslash \mathrm{H}, 3.2672585362,1.2090019689,-1.4103992351 \backslash \mathrm{H}, 2.0741851568,1.306$ $6396958,-2.7237194973 \backslash \mathrm{H}, 1.7475289947,2.1013873636,-1.1629932337 \backslash \backslash V e r s i$ on=x86-Linux-G98RevA.11.1 \State=1-A ' $\backslash \mathrm{HF}=-1071.1171695 \backslash \mathrm{RMSD}=5.909 e-09 \backslash \mathrm{R}$ $\mathrm{MSF}=4.928 e-06 \backslash \mathrm{Dipole}=-0.0244088,0 ., 1.1448754 \backslash \mathrm{PG}=\mathrm{CS} \quad[\mathrm{SG}(\mathrm{C} 1 \mathrm{H} 1 \mathrm{~N} 2 \mathrm{P} 2), \mathrm{X}(\mathrm{C} 6 \mathrm{H}$ 20) $] \backslash \backslash Q$

\section{Single Point Energy Calculation of Planar $\mathrm{Me}_{3} \mathrm{P}-\mathrm{P}\left(\mathrm{NMe}_{2}\right)_{2}{ }^{+}$in $C_{s}$ symmetry}

$1 \backslash 1 \backslash G I N C-N E I L \backslash S P \backslash R B 3 P W 91 \backslash 6-311+G(3 d f, 2 p) \backslash C 7 H 21 N 2 P 2(1+) \backslash B O B B Y \backslash 14-N o v-20$ $03 \backslash 0 \backslash \backslash \#$ B3PW91/6-311+G(3DF, 2P) SCF=TIGHT \#P GFINPUT IOP (6/7=3) TEST \\S ingle Point and Molden of Me3P-P(NMe2)2+-planar \\1,1\P,0,0.,0.47699,0. $\backslash \mathrm{P}, 0,0.035605,-1.607967,0 . \backslash \mathrm{C}, 0,1.776435,-2.123957,0 . \backslash \mathrm{C}, 0,-0.775378,-2$. $360076,1.450802 \backslash \mathrm{C}, 0,-0.775378,-2.360076,-1.450802 \backslash \mathrm{H}, 0,1.818346,-3.2181$ $4,0 . \backslash \mathrm{H}, 0,2.283005,-1.744406,-0.890442 \backslash \mathrm{H}, 0,2.283005,-1.744406,0.890442 \backslash$ $\mathrm{H}, 0,-1.811857,-2.015564,1.510511 \backslash \mathrm{H}, 0,-0.768886,-3.452063,1.361106 \backslash \mathrm{H}, 0$, $-0.24904,-2.06162,2.361267 \backslash \mathrm{H}, 0,-1.811857,-2.015564,-1.510511 \backslash \mathrm{H}, 0,-0.24$ $904,-2.06162,-2.361267 \backslash \mathrm{H}, 0,-0.768886,-3.452063,-1.361106 \backslash \mathrm{N}, 0,-1.566202$ $, 1.061562,0 . \backslash \mathrm{N}, 0,1.472132,1.247127,0 . \backslash \mathrm{C}, 0,-2.154025,1.623937,-1.22335 \backslash$ $\mathrm{C}, 0,-2.154025,1.623937,1.22335 \backslash \mathrm{C}, 0,2.046908,1.816645,-1.225338 \backslash \mathrm{C}, 0,2.0$ $46908,1.816645,1.225338 \backslash \mathrm{H}, 0,-3.238625,1.467304,-1.201089 \backslash \mathrm{H}, 0,-1.965875$

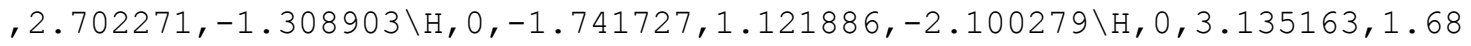
$3733,-1.209002 \backslash \mathrm{H}, 0,1.642049,1.307702,-2.101387 \backslash \mathrm{H}, 0,1.834497,2.89059,-1$ $.30664 \backslash \mathrm{H}, 0,-3.238625,1.467304,1.201089 \backslash \mathrm{H}, 0,-1.741727,1.121886,2.100279$ $\backslash \mathrm{H}, 0,-1.965875,2.702271,1.308903 \backslash \mathrm{H}, 0,3.135163,1.683733,1.209002 \backslash \mathrm{H}, 0,1$. $834497,2.89059,1.30664 \backslash \mathrm{H}, 0,1.642049,1.307702,2.101387 \backslash \backslash$ Version=x86-Lin

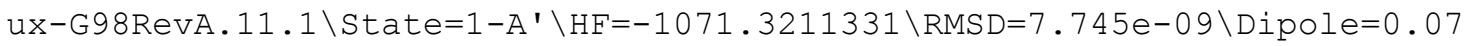
$31414,-1.0724788,0 . \backslash \mathrm{PG}=\mathrm{CS} \quad[\mathrm{SG}(\mathrm{C} 1 \mathrm{H} 1 \mathrm{~N} 2 \mathrm{P} 2), \mathrm{X}(\mathrm{C} 6 \mathrm{H} 20)] \backslash \backslash @$

\section{Geometry Optimization of Planar Carbene- $\mathrm{PPh}_{2}{ }^{+}$in $C_{1}$ symmetry}

$1 \backslash 1 \backslash G I N C-N E I L \backslash F T S \backslash R B 3 P W 91 \backslash 6-31 G(d) \backslash C 15 H 14 N 2 P 1(1+) \backslash B O B B Y \backslash 29-N o v-2003 \backslash 0 \backslash$ \\# B3PW91/6-31G(D) OPT=(TS, NOEIGEN) FREQ=NORAMAN POP=(FULL, NBO) TEST \ 
Geom and Freq of Carb-PPh2+-planar-ts \\1,1\P,0.0578952096,-0.000097084 $1,-0.0005875557 \backslash \mathrm{C}, 1.8044758976,-0.0030065344,-0.0177140775 \backslash \mathrm{C},-0.711908$ $3179,-1.6214895859,-0.1016688347 \backslash C,-0.7043731047,1.6238431064,0.115552$ $0053 \backslash \mathrm{C},-0.8069656052,-2.4301996869,1.0456210245 \backslash \mathrm{C},-1.2356008585,-2.076$ $8779524,-1.3247443995 \backslash \mathrm{C},-1.4039704761,-3.6845783983,0.9600537903 \backslash \mathrm{C},-1$. $845784782,-3.3256264748,-1.3937549656 \backslash C,-1.9258169419,-4.1284724668,-0$ $.2554179895 \backslash \mathrm{C},-1.2026172792,2.0808372092,1.3486182916 \backslash \mathrm{C},-0.819036852,2$ $.433006571,-1.0296253018 \backslash \mathrm{C},-1.8071522562,3.3316134191,1.4295634865 \backslash \mathrm{C},-$ $1.4100422065,3.6893748173,-0.9323793435 \backslash C,-1.9066318421,4.1348666973,0$ $.2930466581 \backslash \mathrm{H},-0.422128827,-2.0717417144,1.9958088295 \backslash \mathrm{H},-1.1660591479$, $-1.4529079089,-2.2105274032 \backslash \mathrm{H},-1.47482636,-4.3092606725,1.8457968453 \backslash \mathrm{H}$ $,-2.2552608984,-3.6736847305,-2.3375836561 \backslash \mathrm{H},-2.4010383761,-5.10364255$ $14,-0.3147437101 \backslash \mathrm{H},-1.1179241985,1.4565344937,2.232846205 \backslash \mathrm{H},-0.4540018$ $746,2.073364737,-1.9871511513 \backslash \mathrm{H},-2.1970070602,3.6809263752,2.381204665$ $1 \backslash \mathrm{H},-1.4960326753,4.3144014484,-1.8165381207 \backslash \mathrm{H},-2.3773282137,5.1116205$ $875,0.3616673655 \backslash \mathrm{N}, 2.6288032585,1.0647377216,0.1029108825 \backslash \mathrm{N}, 2.62271788$ $14,-1.0734880429,-0.1544432165 \backslash \mathrm{C}, 3.9567381081,0.6646184156,0.041110699$ $3 \backslash \mathrm{C}, 3.9529353523,-0.6778018011,-0.1187049266 \backslash \mathrm{H}, 2.3029802994,2.01346685$ $08,0.2258977054 \backslash \mathrm{H}, 2.2913862749,-2.0211206895,-0.2709976129 \backslash \mathrm{H}, 4.7702315$ $621,1.36870854,0.1166786441 \backslash \mathrm{H}, 4.7624403578,-1.3846002658,-0.2101556322$

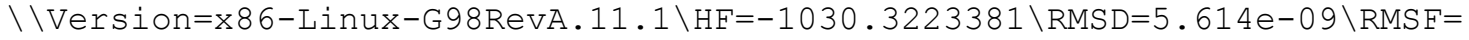
$2.113 e-06 \backslash \mathrm{Dipole}=1.8308697,-0.0030829,-0.0179409 \backslash \mathrm{PG}=\mathrm{C} 01 \quad[\mathrm{X}(\mathrm{C} 15 \mathrm{H} 14 \mathrm{~N} 2 \mathrm{P} 1)$ ]$\backslash \backslash$ Q

\section{Single Point Energy Calculation of Planar Carbene- $\mathrm{PPh}_{2}{ }^{+}$in $C_{1}$ symmetry}

$1 \backslash 1 \backslash G I N C-N E I L \backslash S P \backslash R B 3 P W 91 \backslash 6-311+G(3 d f, 2 p) \backslash C 15 H 14 N 2 P 1(1+) \backslash B O B B Y \backslash 03-D e c-2$ $003 \backslash 0 \backslash \backslash \#$ B3PW91/6-311+G(3DF, 2P) SCF=TIGHT \#P GFINPUT IOP $(6 / 7=3)$ TEST \

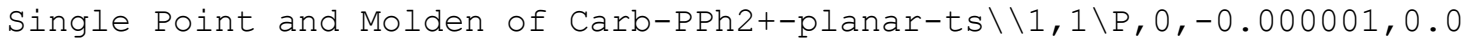
$57898,0.000018 \backslash \mathrm{C}, 0,0.000022,1.804565,-0.000025 \backslash \mathrm{C}, 0,-1.626231,-0.708153$ $, 0.016419 \backslash \mathrm{C}, 0,1.626206,-0.708198,-0.01638 \backslash \mathrm{C}, 0,-2.368776,-0.813128,-1.1$ $73929 \backslash \mathrm{C}, 0,-2.151426,-1.219032,1.216719 \backslash \mathrm{C}, 0,-3.627319,-1.407157,-1.1538$ $72 \backslash \mathrm{C}, 0,-3.403438,-1.826412,1.220694 \backslash \mathrm{C}, 0,-4.140603,-1.916284,0.03947 \backslash \mathrm{C}$, $0,2.15129,-1.219306,-1.21663 \backslash \mathrm{C}, 0,2.368856,-0.812956,1.173923 \backslash \mathrm{C}, 0,3.403$ $291,-1.826705,-1.220598 \backslash \mathrm{C}, 0,3.627395,-1.406996,1.153867 \backslash \mathrm{C}, 0,4.140565,-$ $1.916355,-0.039424 \backslash \mathrm{H}, 0,-1.956143,-0.438252,-2.105943 \backslash \mathrm{H}, 0,-1.578548,-1$. $141833,2.13576 \backslash \mathrm{H}, 0,-4.200913,-1.485669,-2.072901 \backslash \mathrm{H}, 0,-3.805382,-2.2260$ $06,2.147189 \backslash \mathrm{H}, 0,-5.118626,-2.389263,0.047987 \backslash \mathrm{H}, 0,1.578335,-1.142262,-2$ $.135637 \backslash \mathrm{H}, 0,1.956299,-0.437923,2.105908 \backslash \mathrm{H}, 0,3.805146,-2.226482,-2.1470$ $53 \backslash \mathrm{H}, 0,4.201073,-1.485339,2.072858 \backslash \mathrm{H}, 0,5.118585,-2.389341,-0.047936 \backslash \mathrm{N}$, $0,1.074727,2.625875,-0.06788 \backslash \mathrm{N}, 0,-1.074662,2.625907,0.067788 \backslash \mathrm{C}, 0,0.674$ $712,3.955022,-0.041791 \backslash \mathrm{C}, 0,-0.674616,3.955043,0.04163 \backslash \mathrm{H}, 0,2.028175,2.2$ $97267,-0.133667 \backslash \mathrm{H}, 0,-2.028117,2.297327,0.133602 \backslash \mathrm{H}, 0,1.38377,4.766551,-$ $0.085192 \backslash \mathrm{H}, 0,-1.383655,4.766592,0.084958 \backslash \backslash$ Version=x86-Linux-G98RevA. 11 $.1 \backslash \mathrm{HF}=-1030.5646037 \backslash \mathrm{RMSD}=8.727 e-09 \backslash \mathrm{Dipole}=-0.0000114,1.6604936,-0.0000$ $546 \backslash \mathrm{PG}=\mathrm{C} 01 \quad[\mathrm{X}(\mathrm{C} 15 \mathrm{H} 14 \mathrm{~N} 2 \mathrm{P} 1)] \backslash \backslash @$

\section{Geometry Optimization of Planar $\mathrm{Me}_{3} \mathrm{P}-\mathrm{PPh}_{2}{ }^{+}$in $C_{1}$ symmetry}

$1 \backslash 1 \backslash G I N C-N E I L \backslash F T S \backslash R B 3 P W 91 \backslash 6-31 G(d) \backslash C 15 H 19 P 2(1+) \backslash B O B B Y \backslash 21-D e C-2003 \backslash 0 \backslash \backslash \#$ B3PW91/6-31G (D) OPT=(TS, NOEIGEN) FREQ=NORAMAN POP=(FULL, NBO) TEST \\Ge om and Freq of Me3P-PPh2+-planar-ts \\1,1\P,0.1030555557,-0.0042815285, $-0.0008965638 \backslash P, 2.2050269673,0.0346228832,0.0146936494 \backslash C,-0.696772403$, 
$-1.6001284031,-0.2856009889 \backslash \mathrm{C},-0.7229851486,1.5780799768,0.2757844686 \backslash$ $\mathrm{C},-1.0285908684,-2.4260530041,0.8020016055 \backslash \mathrm{C},-1.015865819,-2.005134675$ $,-1.5929969394 \backslash \mathrm{C},-1.6675850806,-3.6425024879,0.5791193175 \backslash \mathrm{C},-1.6547495$ $36,-3.2236209713,-1.8048358256 \backslash \mathrm{C},-1.9780381046,-4.0406970959,-0.721332$ $2783 \backslash \mathrm{C},-1.0577224951,1.9810394568,1.5802109714 \backslash \mathrm{C},-1.0426915672,2.40728$ $20589,-0.8133283358 \backslash \mathrm{C},-1.6982705556,3.1992706973,1.7876812189 \backslash \mathrm{C},-1.683$ $053178,3.6237700804,-0.5953016648 \backslash \mathrm{C},-2.0085947757,4.018991257,0.702368$ $3087 \backslash \mathrm{H},-0.793719336,-2.10946869,1.8135529326 \backslash \mathrm{H},-0.771265233,-1.3629437$ $82,-2.4334646415 \backslash \mathrm{H},-1.9264470562,-4.2777414067,1.4213514353 \backslash \mathrm{H},-1.90366$ $94628,-3.5332168995,-2.8158171507 \backslash \mathrm{H},-2.478219443,-4.9901314692,-0.8908$ $512773 \backslash \mathrm{H},-0.8221559547,1.3375175046,2.4223994121 \backslash \mathrm{H},-0.7955153388,2.093$ $4019001,-1.8229128762 \backslash \mathrm{H},-1.9584018692,3.5069985845,2.7964066366 \backslash \mathrm{H},-1.9$ $313879875,4.2614269163,-1.438876116 \backslash \mathrm{H},-2.5099419545,4.9684555195,0.868$ $2968569 \backslash \mathrm{C}, 2.9140727756,0.6078760801,1.594065597 \backslash \mathrm{C}, 2.7799907524,-1.6643$ $32685,-0.2781107187 \backslash \mathrm{C}, 2.9272041984,1.1054290323,-1.2728959538 \backslash \mathrm{H}, 2.5272$ $31793,1.6038533384,1.8301064091 \backslash \mathrm{H}, 2.6247070391,-0.080505682,2.39253024$ $35 \backslash \mathrm{H}, 4.0062826549,0.6574549183,1.5248895242 \backslash \mathrm{H}, 2.4151043647,-2.32816498$ $74,0.5096372813 \backslash \mathrm{H}, 2.4176531519,-2.0251270954,-1.243997246 \backslash \mathrm{H}, 3.87478906$ $,-1.6696455716,-0.2774972157 \backslash \mathrm{H}, 4.0187523848,1.1264986879,-1.1820021821$ $\backslash \mathrm{H}, 2.6453183763,0.7271219235,-2.2591039813 \backslash \mathrm{H}, 2.5415577991,2.1234800656$ ,$-1.1625770228 \backslash \backslash V e r s i o n=x 86-L i n u x-G 98 R e v A .11 .1 \backslash H F=-1265.2037627 \backslash R M S D=5$ $.230 e-09 \backslash \mathrm{RMSF}=3.895 e-06 \backslash \mathrm{Dipole}=2.0587567,-0.0244498,0.0049786 \backslash \mathrm{PG}=\mathrm{C} 01 \quad[$ $\mathrm{X}(\mathrm{C} 15 \mathrm{H} 19 \mathrm{P} 2) \mathrm{]} \backslash \backslash \mathrm{Q}$

\section{Single Point Energy Calculation of Planar $\mathrm{Me}_{3} \mathrm{P}-\mathrm{PPh}_{2}{ }^{+}$in $C_{1}$ symmetry}

$1 \backslash 1 \backslash G I N C-N E I L \backslash S P \backslash R B 3 P W 91 \backslash 6-311+G(3 d f, 2 p) \backslash C 15 H 19 P 2(1+) \backslash B O B B Y \backslash 23-D e c-200$ $3 \backslash 0 \backslash \backslash \#$ B3PW91/6-311+G(3DF,2P) SCF=TIGHT \#P GFINPUT IOP (6/7=3) TEST \\i ngle Point and Molden of Me3P-PPh2+-planar-ts \\1,1\P,0,-0.003475,0.103 $089,0.000463 \backslash \mathrm{P}, 0,0.055838,2.20464,-0.001507 \backslash \mathrm{C}, 0,-1.631416,-0.682607,0$. $000728 \backslash \mathrm{C}, 0,1.595642,-0.736904,0.000636 \backslash \mathrm{C}, 0,-2.258403,-1.005157,-1.2149$ $96 \backslash \mathrm{C}, 0,-2.260443,-1.000068,1.216737 \backslash \mathrm{C}, 0,-3.500591,-1.633387,-1.209112 \backslash$ $\mathrm{C}, 0,-3.502715,-1.628151,1.211392 \backslash \mathrm{C}, 0,-4.121628,-1.942258,0.001271 \backslash \mathrm{C}, 0$, $2.216448,-1.073233,-1.214863 \backslash \mathrm{C}, 0,2.219924,-1.065884,1.216365 \backslash \mathrm{C}, 0,3.446$ $575,-1.724531,-1.209288 \backslash \mathrm{C}, 0,3.450173,-1.716963,1.211243 \backslash \mathrm{C}, 0,4.062263,-$ $2.044036,0.001081 \backslash \mathrm{H}, 0,-1.768644,-0.771578,-2.155316 \backslash \mathrm{H}, 0,-1.772149,-0.7$ $62665,2.156861 \backslash \mathrm{H}, 0,-3.981858,-1.885111,-2.149809 \backslash \mathrm{H}, 0,-3.985616,-1.8758$ $41,2.152323 \backslash \mathrm{H}, 0,-5.090392,-2.434031,0.001487 \backslash \mathrm{H}, 0,1.73132,-0.830474,-2$. $155402 \backslash \mathrm{H}, 0,1.737365,-0.817457,2.156737 \backslash \mathrm{H}, 0,3.922814,-1.985857,-2.14992$ $4 \backslash \mathrm{H}, 0,3.929173,-1.972438,2.152086 \backslash \mathrm{H}, 0,5.021716,-2.553755,0.001254 \backslash \mathrm{C}, 0$, $0.901247,2.910934,-1.454853 \backslash \mathrm{C}, 0,-1.663081,2.794604,-0.006873 \backslash \mathrm{C}, 0,0.892$ $571,2.914982,1.454977 \backslash \mathrm{H}, 0,1.919696,2.515403,-1.515272 \backslash \mathrm{H}, 0,0.359771,2.6$ $29122,-2.361789 \backslash \mathrm{H}, 0,0.947539,4.002535,-1.37467 \backslash \mathrm{H}, 0,-2.182904,2.437035$, $-0.899224 \backslash \mathrm{H}, 0,-2.189539,2.43404,0.880388 \backslash \mathrm{H}, 0,-1.658678,3.889405,-0.004$ $956 \backslash \mathrm{H}, 0,0.938629,4.006436,1.372569 \backslash \mathrm{H}, 0,0.346032,2.634994,2.359457 \backslash \mathrm{H}, 0$, $1.910895,2.520246,1.522205 \backslash \backslash$ Version=x86-Linux-G98RevA $.11 .1 \backslash H F=-1265.44$ $68054 \backslash \mathrm{RMSD}=5.359 \mathrm{e}-09 \backslash \mathrm{Dipole}=-0.0113239,1.9556937,-0.0025172 \backslash \mathrm{PG}=\mathrm{C} 01] \mathrm{X}($ C15H19P2) ] \\@

\section{SIMPLE P ORBITAL OCCUPANCY CALCULATIONS}

\section{Geometry Optimization of $\mathrm{PH}_{2}{ }^{+}$in $C_{2 v}$ symmetry}

$1 \backslash 1 \backslash G I N C-N E I L \backslash F O p t \backslash R B 3 P W 91 \backslash 6-31 G(d) \backslash H 2 P 1(1+) \backslash C H U C K \backslash 15-J u n-2004 \backslash 0 \backslash \backslash \#$ B3 
PW91/6-31G(D) OPT POP=(FULL, NBO) TEST \\DFT OPTIMIZATION OF PH2 \1, $1 \backslash \mathrm{P}$, $-0.0892532101,0 .,-0.0748923357 \backslash \mathrm{H}, 0.0087425653,0.1 .3490322871 \backslash \mathrm{H}, 1.3300$ $555859,0 .,-0.2256472522 \backslash \backslash$ Version=x86-Linux-G98RevA.11.1 $\backslash$ State $=1-\mathrm{A} 1 \backslash \mathrm{HF}=$ $-342.0851386 \backslash \mathrm{RMSD}=6.915 e-09 \backslash \mathrm{RMSF}=3.468 e-05 \backslash \mathrm{Dipole}=0.3195723,0 ., 0.26815$ $3 \backslash \mathrm{PG}=\mathrm{C} 02 \mathrm{~V} \quad[\mathrm{C} 2(\mathrm{P} 1), \mathrm{SGV}(\mathrm{H} 2)] \backslash \backslash \mathrm{C}$

\section{Geometry Optimization of $\mathrm{PMe}_{2}{ }^{+}$in $C_{2}$ symmetry}

$1 \backslash 1 \backslash \mathrm{GINC}-\mathrm{NEIL} \backslash \mathrm{FOpt} \backslash \mathrm{RB} 3 \mathrm{PW} 91 \backslash 6-31 \mathrm{G}(\mathrm{d}) \backslash \mathrm{C} 2 \mathrm{H} 6 \mathrm{P} 1(1+) \backslash \mathrm{BOBBY} \backslash 11-\mathrm{Nov}-2003 \backslash 1 \backslash \backslash \#$ B3PW91/6-31G (D) FOPT=Z-MATRIX FREQ=NORAMAN POP=(FULL, NBO) TEST \\Geom a

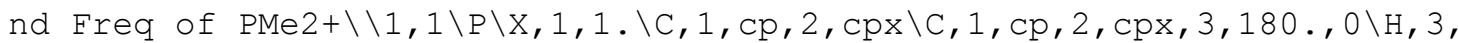
$\mathrm{hc1}, 1, \mathrm{hcp} 1,2, \mathrm{~d} 1,0 \backslash \mathrm{H}, 4, \mathrm{hcl}, 1, \mathrm{hcp} 1,2, \mathrm{~d} 1,0 \backslash \mathrm{H}, 3, \mathrm{hc} 2,1, \mathrm{hcp} 2,5, \mathrm{~d} 2,0 \backslash \mathrm{H}, 4, \mathrm{hc} 2$,

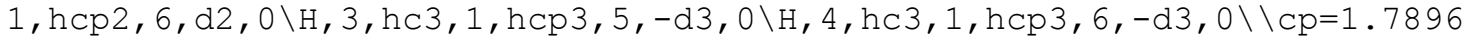
$1592 \backslash \mathrm{cpx}=128.2843412 \backslash \mathrm{hc1}=1.09623548 \backslash \mathrm{hcp} 1=111.66188124 \backslash \mathrm{hc} 2=1.09487306 \backslash \mathrm{h}$ $\mathrm{cp} 2=116.31984005 \backslash \mathrm{hc} 3=1.11528864 \backslash \mathrm{hcp} 3=103.13637665 \backslash \mathrm{d} 1=27.47225317 \backslash \mathrm{d} 2=13$ $1.15108299 \backslash \mathrm{d} 3=112.07135692 \backslash \backslash$ Version=x86-Linux-G98RevA.11.1 $\backslash$ State $=1-\mathrm{A} \backslash \mathrm{H}$ $\mathrm{F}=-420.7722914 \backslash \mathrm{RMSD}=2.691 e-09 \backslash \mathrm{RMSF}=3.716 e-05 \backslash \mathrm{Dipole}=0 ., 0 .,-0.2924307 \backslash \mathrm{P}$ $\mathrm{G}=\mathrm{C} 02 \quad[\mathrm{C} 2(\mathrm{P} 1), \mathrm{X}(\mathrm{C} 2 \mathrm{H} 6)] \backslash \backslash @$

\section{TRIPLET ENERGY CALCULATIONS}

\section{Geometry Optimization of triplet $\mathrm{PPh}_{2}^{+}$in $C_{2}$ symmetry}

$1 \backslash 1 \backslash G I N C-N E I L \backslash F O p t \backslash U B 3 P W 91 \backslash 6-31 G(d) \backslash C 12 H 10 P 1(1+, 3) \backslash B O B B Y \backslash 24-J u 1-2004 \backslash 0$ $\backslash \backslash \#$ UB3PW91/6-31G(D) OPT FREQ=NORAMAN POP=(FULL, NBO) TEST \\Geom and Fr

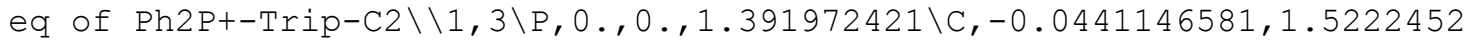
$736,0.4630616714 \backslash \mathrm{C}, 0.0441146581,-1.5222452736,0.4630616714 \backslash \mathrm{C}, 0.8068127$ $628,2.5859909275,0.8459886302 \backslash \mathrm{C},-0.8068127628,-2.5859909275,0.84598863$ $02 \backslash \mathrm{C},-0.9883856007,1.6945550632,-0.5790420924 \backslash \mathrm{C}, 0.9883856007,-1.694555$ $0632,-0.5790420924 \backslash \mathrm{C}, 0.7684103259,3.7753479601,0.135670083 \backslash \mathrm{C},-0.768410$ $3259,-3.7753479601,0.135670083 \backslash \mathrm{C},-0.9982293911,2.8847304931,-1.2864093$ $286 \backslash \mathrm{C}, 0.9982293911,-2.8847304931,-1.2864093286 \backslash \mathrm{C},-0.1298667042,3.92612$ $52596,-0.9276411101 \backslash \mathrm{C}, 0.1298667042,-3.9261252596,-0.9276411101 \backslash \mathrm{H}, 1.499$ $7251254,2.4636689485,1.673368551 \backslash \mathrm{H},-1.4997251254,-2.4636689485,1.67336$ $8551 \backslash \mathrm{H},-1.6732454697,0.8939914775,-0.840695925 \backslash \mathrm{H}, 1.6732454697,-0.89399$ $14775,-0.840695925 \backslash \mathrm{H}, 1.4359013044,4.5878112843,0.4057913685 \backslash \mathrm{H},-1.43590$ $13044,-4.5878112843,0.4057913685 \backslash \mathrm{H},-1.6893058176,3.0130299613,-2.11424$ $02784 \backslash \mathrm{H}, 1.6893058176,-3.0130299613,-2.1142402784 \backslash \mathrm{H},-0.1638394855,4.864$ $4874361,-1.4737839947 \backslash \mathrm{H}, 0.1638394855,-4.8644874361,-1.4737839947 \backslash \backslash$ Vers

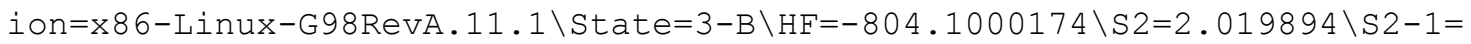
$0 . \backslash \mathrm{S} 2 \mathrm{~A}=2.000231 \backslash \mathrm{RMSD}=9.204 \mathrm{e}-09 \backslash \mathrm{RMSF}=8.642 e-06 \backslash \mathrm{Dipole}=0 ., 0 .,-0.2956122 \backslash$ $\mathrm{PG}=\mathrm{C} 02 \quad[\mathrm{C} 2(\mathrm{P} 1), \mathrm{X}(\mathrm{C} 12 \mathrm{H} 10)] \backslash \backslash \mathrm{Q}$

\section{Single Point Calculation of triplet $\mathrm{PPh}_{2}^{+}$in $C_{2}$ symmetry}

$1 \backslash 1 \backslash G I N C-N E I L \backslash S P \backslash U B 3 P W 91 \backslash 6-311+G(3 \mathrm{df}, 2 \mathrm{p}) \backslash \mathrm{C} 12 \mathrm{H} 10 \mathrm{P} 1(1+, 3) \backslash \mathrm{BOBBY} \backslash 25-\mathrm{Jul}-2$ $004 \backslash 0 \backslash \backslash \#$ UB3PW91/6-311+G(3DF, 2P) SCF=TIGHT \#P GFINPUT IOP $(6 / 7=3)$ TEST $\backslash$ Single Point and Molden of Ph2P+-Trip-C2\\1,3\P,0,0.,0.,1.391972\C,0, $0 ., 1.522884,0.463062 \backslash \mathrm{C}, 0,0 .,-1.522884,0.463062 \backslash \mathrm{C}, 0,0.881385,2.561534,0$ $.845989 \backslash \mathrm{C}, 0,-0.881385,-2.561534,0.845989 \backslash \mathrm{C}, 0,-0.938883,1.722475,-0.579$ $042 \backslash \mathrm{C}, 0,0.938883,-1.722475,-0.579042 \backslash \mathrm{C}, 0,0.877452,3.751504,0.13567 \backslash \mathrm{C}, 0$ $,-0.877452,-3.751504,0.13567 \backslash \mathrm{C}, 0,-0.914246,2.912436,-1.286409 \backslash \mathrm{C}, 0,0.91$ $4246,-2.912436,-1.286409 \backslash \mathrm{C}, 0,-0.016081,3.92824,-0.927641 \backslash \mathrm{C}, 0,0.016081$, $-3.92824,-0.927641 \backslash \mathrm{H}, 0,1.570463,2.419191,1.673369 \backslash \mathrm{H}, 0,-1.570463,-2.419$ $191,1.673369 \backslash \mathrm{H}, 0,-1.646646,0.942087,-0.840696 \backslash \mathrm{H}, 0,1.646646,-0.942087,-$ 
$0.840696 \backslash \mathrm{H}, 0,1.568198,4.544291,0.405791 \backslash \mathrm{H}, 0,-1.568198,-4.544291,0.4057$ $91 \backslash \mathrm{H}, 0,-1.601316,3.060701,-2.11424 \backslash \mathrm{H}, 0,1.601316,-3.060701,-2.11424 \backslash \mathrm{H}, 0$ $,-0.022857,4.867192,-1.473784 \backslash \mathrm{H}, 0,0.022857,-4.867192,-1.473784 \backslash \backslash$ Versio $\mathrm{n}=\mathrm{x} 86-\mathrm{Linux}-\mathrm{G} 98 \mathrm{RevA} .11 .1 \backslash \mathrm{State}=3-\mathrm{B} \backslash \mathrm{HF}=-804.2617971 \backslash \mathrm{S} 2=2.02098 \backslash \mathrm{S} 2-1=0 . \backslash$ $\mathrm{S} 2 \mathrm{~A}=2.000257 \backslash \mathrm{RMSD}=6.262 \mathrm{e}-09 \backslash \mathrm{Dipole}=0 ., 0 .,-0.2222954 \backslash \mathrm{PG}=\mathrm{C} 02 \quad[\mathrm{C} 2(\mathrm{P} 1), \mathrm{X}(\mathrm{C}$ $12 \mathrm{H} 10)] \backslash \backslash \mathrm{e}$

\section{Geometry Optimization of triplet $\mathrm{P}\left(\mathrm{NMe}_{2}\right)_{2}{ }^{+}$in $C_{2}$ symmetry}

$1 \backslash 1 \backslash G I N C-N E I L \backslash F O p t \backslash U B 3 P W 91 \backslash 6-31 G(d) \backslash C 4 H 12 N 2 P 1(1+, 3) \backslash B O B B Y \backslash 24-J u 1-2004 \backslash$ $0 \backslash \backslash \#$ UB3PW91/6-31G(D) OPT FREQ=NORAMAN POP=(FULL, NBO) TEST \Geom and $F$ req of $(\mathrm{Me} 2 \mathrm{~N}) 2 \mathrm{P}+-\mathrm{Trip}-\mathrm{C} 2 \backslash \backslash 1,3 \backslash \mathrm{P}, 0 ., 0 ., 1.0399844884 \backslash \mathrm{N},-0.0753876974,1.3$ $294918085,-0.0863425331 \backslash N, 0.0753876974,-1.3294918085,-0.0863425331 \backslash \mathrm{C},-$ $0.7631773173,2.5365799759,0.3545837864 \backslash \mathrm{C}, 0.7631773173,-2.5365799759,0$. $3545837864 \backslash \mathrm{C}, 0.9515982321,1.5116726824,-1.1011765957 \backslash \mathrm{C},-0.9515982321,-$ $1.5116726824,-1.1011765957 \backslash \mathrm{H},-1.5338365056,2.2871809852,1.0868832133 \backslash \mathrm{H}$ $, 1.5338365056,-2.2871809852,1.0868832133 \backslash \mathrm{H}, 1.2288711142,0.5440694117,-$ $1.5272491677 \backslash \mathrm{H},-1.2288711142,-0.5440694117,-1.5272491677 \backslash \mathrm{H},-0.06027346$ $29,3.2575385675,0.7926885593 \backslash \mathrm{H}, 0.0602734629,-3.2575385675,0.7926885593$ $\backslash \mathrm{H},-1.2286230819,3.0063237094,-0.5201023832 \backslash \mathrm{H}, 1.2286230819,-3.00632370$ $94,-0.5201023832 \backslash \mathrm{H}, 0.5690364625,2.1767135224,-1.8817067946 \backslash \mathrm{H},-0.569036$ $4625,-2.1767135224,-1.8817067946 \backslash \mathrm{H}, 1.8513935842,1.9696652945,-0.666442$ $5026 \backslash \mathrm{H},-1.8513935842,-1.9696652945,-0.6664425026 \backslash \backslash$ Version=x86-Linux-G9

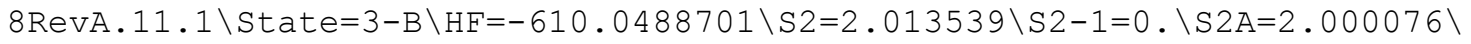
$\mathrm{RMSD}=3.966 \mathrm{e}-09 \backslash \mathrm{RMSF}=2.797 e-06 \backslash \mathrm{Dipole}=0 ., 0 .,-0.5533939 \backslash \mathrm{PG}=\mathrm{C} 02[\mathrm{C} 2(\mathrm{P} 1), \mathrm{X}$ (C4H12N2) ] \\@

\section{Single Point Calculation of triplet $\mathrm{P}\left(\mathrm{NMe}_{2}\right)_{2}{ }^{+}$in $C_{2}$ symmetry}

$1 \backslash 1 \backslash G I N C-N E I L \backslash S P \backslash U B 3 P W 91 \backslash 6-311+G(3 d f, 2 p) \backslash C 4 H 12 N 2 P 1(1+, 3) \backslash B O B B Y \backslash 25-J u 1-$ $2004 \backslash 0 \backslash \backslash \#$ UB3PW91/6-311+G(3DF, 2P) SCF=TIGHT \#P GFINPUT IOP (6/7=3) TEST $\backslash \backslash$ Single Point and Molden of (Me2N) 2P+-Trip-C2\\1,3\P,0,0.,0.,1.039984 $\backslash \mathrm{N}, 0,0.1 .331627,-0.086343 \backslash \mathrm{N}, 0,0 .,-1.331627,-0.086343 \backslash \mathrm{C}, 0,-0.618349,2$. $575718,0.354584 \backslash \mathrm{C}, 0,0.618349,-2.575718,0.354584 \backslash \mathrm{C}, 0,1.035653,1.455375$, $-1.101177 \backslash \mathrm{C}, 0,-1.035653,-1.455375,-1.101177 \backslash \mathrm{H}, 0,-1.401892,2.370348,1.0$ $86883 \backslash \mathrm{H}, 0,1.401892,-2.370348,1.086883 \backslash \mathrm{H}, 0,1.257702,0.473626,-1.527249 \backslash$ $\mathrm{H}, 0,-1.257702,-0.473626,-1.527249 \backslash \mathrm{H}, 0,0.124243,3.255726,0.792689 \backslash \mathrm{H}, 0,-$ $0.124243,-3.255726,0.792689 \backslash \mathrm{H}, 0,-1.056455,3.071058,-0.520102 \backslash \mathrm{H}, 0,1.056$ $455,-3.071058,-0.520102 \backslash \mathrm{H}, 0,0.691355,2.141008,-1.881707 \backslash \mathrm{H}, 0,-0.691355$, $-2.141008,-1.881707 \backslash \mathrm{H}, 0,1.959933,1.861693,-0.666443 \backslash \mathrm{H}, 0,-1.959933,-1.8$ $61693,-0.666443 \backslash \backslash$ Version=x86-Linux-G98RevA.11.1 \State=3-B \HF=-610.1686 $411 \backslash S 2=2.016715 \backslash S 2-1=0 . \backslash S 2 A=2.000123 \backslash R M S D=3.836 e-09 \backslash D i p o l e=0,0 .,-0.55$ $21117 \backslash \mathrm{PG}=\mathrm{C} 02 \quad[\mathrm{C} 2(\mathrm{P} 1), \mathrm{X}(\mathrm{C} 4 \mathrm{H} 12 \mathrm{~N} 2)] \backslash \backslash \mathrm{Q}$

\section{PROTON AFFINITY CALCULATIONS}

\section{Geometry Optimization of $\mathrm{HPMe}_{3}{ }^{+}$in $C_{3}$ symmetry}

$1 \backslash 1 \backslash G I N C-N E I L \backslash F O p t \backslash R B 3 P W 91 \backslash 6-31 G(d) \backslash C 3 H 10 P 1(1+) \backslash C H U C K \backslash 31-J u 1-2004 \backslash 0 \backslash \backslash \#$ B3PW91/6-31G(D) OPT FREQ=NORAMAN POP=(FULL, NBO) TEST \\Geom and Freq 0 f $\mathrm{PMe} 3-\mathrm{H}$ Cation $\backslash \backslash 1,1 \backslash \mathrm{P}, 0 ., 0 ., 0.3315486087 \backslash \mathrm{H}, 0 ., 0 ., 1.7337829911 \backslash \mathrm{C}, 1.723$ $6670547,0.0578897425,-0.2110053729 \backslash \mathrm{C},-0.8116995397,-1.5216843283,-0.21$ $10053729 \backslash \mathrm{C},-0.911967515,1.4637945858,-0.2110053729 \backslash \mathrm{H}, 2.2635845825,-0.8$ $161630591,0.1659779632 \backslash \mathrm{H},-1.8386102341,-1.5522402225,0.1659779632 \backslash \mathrm{H},-0$ $.4249743484,2.3684032816,0.1659779632 \backslash \mathrm{H}, 1.7690045671,0.0612157845,-1.3$ 
$046700977 \backslash \mathrm{H},-0.831487859,-1.5626107867,-1.3046700977 \backslash \mathrm{H},-0.937516708,1$. $5013950022,-1.3046700977 \backslash \mathrm{H}, 2.2042586685,0.9645981683,0.1690536642 \backslash \mathrm{H},-0$ $.266762816,-2.3912430876,0.1690536642 \backslash \mathrm{H},-1.9374958525,1.4266449193,0.1$ $690536642 \backslash \backslash$ Version=x86-Linux-G98RevA.11. $1 \backslash \mathrm{HF}=-461.3831811 \backslash \mathrm{RMSD}=5.418 \mathrm{e}-$ $09 \backslash \mathrm{RMSF}=1.415 \mathrm{e}-05 \backslash \mathrm{Dipole}=0 ., 0 ., 0.0862672 \backslash \mathrm{PG}=\mathrm{C} 03 \quad[\mathrm{C} 3(\mathrm{P} 1 \mathrm{H} 1), \mathrm{X}(\mathrm{C} 3 \mathrm{H} 9)] \backslash \backslash @$

\section{Single Point Energy Calculation of $\mathrm{HPMe}_{3}{ }^{+}$in $C_{3}$ symmetry}

$1 \backslash 1 \backslash G I N C-N E I L \backslash S P \backslash R B 3 P W 91 \backslash 6-311+G(3 d f, 2 p) \backslash C 3 H 10 P 1(1+) \backslash C H U C K \backslash 31-J u 1-2004$ $\backslash 0 \backslash \backslash \#$ B3PW91/6-311+G(3DF, 2P) GEOM=CHECK GUESS=READ SCF=TIGHT \#P GFINPU

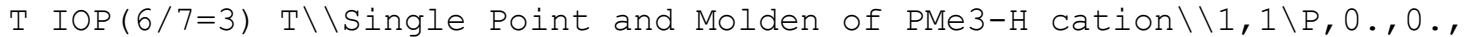
$0.3315486087 \backslash \mathrm{H}, 0.0 .1 .7337829911 \backslash \mathrm{C}, 1.7236670547,0.0578897425,-0.21100$ $53729 \backslash \mathrm{C},-0.8116995397,-1.5216843283,-0.2110053729 \backslash \mathrm{C},-0.911967515,1.463$ $7945858,-0.2110053729 \backslash \mathrm{H}, 2.2635845825,-0.8161630591,0.1659779632 \backslash \mathrm{H},-1.8$ $386102341,-1.5522402225,0.1659779632 \backslash \mathrm{H},-0.4249743484,2.3684032816,0.16$ $59779632 \backslash \mathrm{H}, 1.7690045671,0.0612157845,-1.3046700977 \backslash \mathrm{H},-0.831487859,-1.5$ $626107867,-1.3046700977 \backslash \mathrm{H},-0.937516708,1.5013950022,-1.3046700977 \backslash \mathrm{H}, 2$. $2042586685,0.9645981683,0.1690536642 \backslash \mathrm{H},-0.266762816,-2.3912430876,0.16$ $90536642 \backslash \mathrm{H},-1.9374958525,1.4266449193,0.1690536642 \backslash \backslash$ Version=x86-Linux-

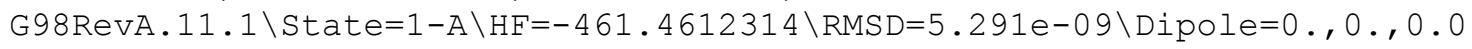
$631188 \backslash \mathrm{PG}=\mathrm{C} 03 \quad[\mathrm{C} 3(\mathrm{P} 1 \mathrm{H} 1), \mathrm{X}(\mathrm{C} 3 \mathrm{H} 9)] \backslash \backslash \mathrm{Q}$

\section{Geometry Optimization of $\mathrm{H}-\mathrm{DHAP}^{+}$in $C_{s}$ symmetry}

$1 \backslash 1 \backslash G I N C-N E I L \backslash F O p t \backslash R B 3 P W 91 \backslash 6-31 G(d) \backslash C 5 H 7 N 2(1+) \backslash$ CHUCK $31-J u l-2004 \backslash 0 \backslash \backslash \#$ B3PW91/6-31G(D) OPT FREQ=NORAMAN POP=(FULL, NBO) TEST \\Geom and Freq of

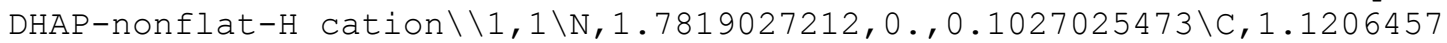
$119,-1.1828334859,0.0643988891 \backslash \mathrm{C}, 1.1206457119,1.1828334859,0.064398889$ $1 \backslash \mathrm{C},-0.2428959597,-1.2157092857,-0.0142158686 \backslash \mathrm{C},-0.2428959597,1.215709$ $2857,-0.0142158686 \backslash \mathrm{C},-0.9803094422,0 \ldots,-0.0562626334 \backslash \mathrm{N},-2.3130763798,0$. $,-0.1329781215 \backslash \mathrm{H},-2.8422144433,0.8602089268,-0.1624952012 \backslash \mathrm{H},-2.8422144$ $433,-0.8602089268,-0.1624952012 \backslash \mathrm{H}, 1.7278785055,-2.0798497747,0.0992999$ $557 \backslash \mathrm{H}, 1.7278785055,2.0798497747,0.0992999557 \backslash \mathrm{H},-0.749063264,-2.1746501$ $689,-0.0438235381 \backslash \mathrm{H},-0.749063264,2.1746501689,-0.0438235381 \backslash \mathrm{H}, 2.793873$ $6403,0 ., 0.1613461404 \backslash \backslash$ Version=x86-Linux-G98RevA.11.1 $\backslash$ State=1-A' $\backslash H F=-30$ $3.922339 \backslash \mathrm{RMSD}=3.675 e-09 \backslash \mathrm{RMSF}=2.903 e-05 \backslash \mathrm{Dipole}=0.1659891,0 ., 0.010871 \backslash \mathrm{PG}$ $=\mathrm{CS} \quad[\mathrm{SG}(\mathrm{C} 1 \mathrm{H} 1 \mathrm{~N} 2), \mathrm{X}(\mathrm{C} 4 \mathrm{H} 6)] \backslash \backslash @$

\section{Single Point Energy Calculation of $\mathrm{H}-\mathrm{DHAP}^{+}$in $C_{s}$ symmetry}

$1 \backslash 1 \backslash G I N C-N E I L \backslash S P \backslash R B 3 P W 91 \backslash 6-311+G(3 d f, 2 p) \backslash C 5 H 7 N 2(1+) \backslash C H U C K \backslash 31-J u 1-2004 \backslash$ $0 \backslash \backslash \#$ B3PW91/6-311+G (3DF, 2P) GEOM=CHECK GUESS=READ SCF=TIGHT \#P GFINPUT

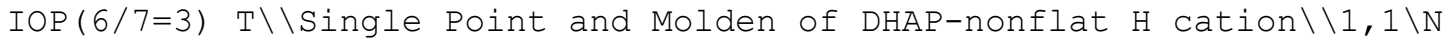
, 1.7819027212,0.,0.1027025473\C,1.1206457119,-1.1828334859,0.064398889 $1 \backslash \mathrm{C}, 1.1206457119,1.1828334859,0.0643988891 \backslash \mathrm{C},-0.2428959597,-1.21570928$ $57,-0.0142158686 \backslash \mathrm{C},-0.2428959597,1.2157092857,-0.0142158686 \backslash \mathrm{C},-0.98030$ $94422,0 \ldots,-0.0562626334 \backslash \mathrm{N},-2.3130763798,0 \ldots,-0.1329781215 \backslash \mathrm{H},-2.842214443$ $3,0.8602089268,-0.1624952012 \backslash \mathrm{H},-2.8422144433,-0.8602089268,-0.16249520$ $12 \backslash \mathrm{H}, 1.7278785055,-2.0798497747,0.0992999557 \backslash \mathrm{H}, 1.7278785055,2.07984977$ $47,0.0992999557 \backslash \mathrm{H},-0.749063264,-2.1746501689,-0.0438235381 \backslash \mathrm{H},-0.749063$ $264,2.1746501689,-0.0438235381 \backslash \mathrm{H}, 2.7938736403,0.0 .1613461404 \backslash \backslash$ Version

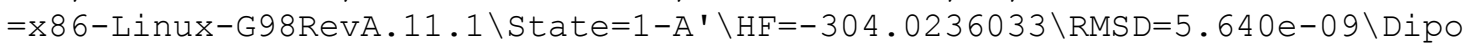
$l e=0.1625616,0 ., 0.010427 \backslash \mathrm{PG}=\mathrm{CS} \quad[\mathrm{SG}(\mathrm{C} 1 \mathrm{H} 1 \mathrm{~N} 2), \mathrm{X}(\mathrm{C} 4 \mathrm{H} 6)] \backslash \backslash \mathrm{C}$

\section{Geometry Optimization of H-Carbene ${ }^{+}$in $C_{2 v}$ symmetry}




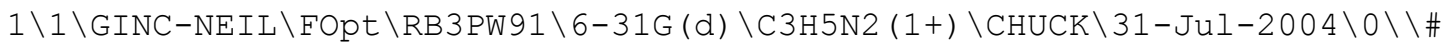
B3PW91/6-31G(D) OPT FREQ=NORAMAN POP=(FULL, NBO) TEST \\Geom and Freq of

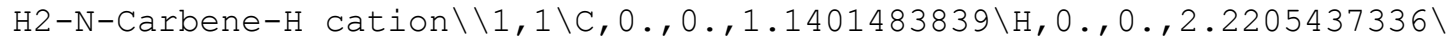
$\mathrm{N}, 1.0710515936,0.0 .3470139332 \backslash \mathrm{N},-1.0710515936,0 \ldots, 0.3470139332 \backslash \mathrm{C}, 0.680$ $9400271,0 .,-0.9749692056 \backslash \mathrm{C},-0.6809400271,0 .,-0.9749692056 \backslash \mathrm{H}, 1.39072583$ $48,0 .,-1.7880133615 \backslash \mathrm{H},-1.3907258348,0 \ldots,-1.7880133615 \backslash \mathrm{H}, 2.0297961505,0$. $, 0.6780140445 \backslash \mathrm{H},-2.0297961505,0 ., 0.6780140445 \backslash \backslash$ Version=x86-Linux-G98Re $\mathrm{VA} .11 .1 \backslash \mathrm{State}=1-\mathrm{A} 1 \backslash \mathrm{HF}=-226.5088149 \backslash \mathrm{RMSD}=9.443 e-09 \backslash \mathrm{RMSF}=1.344 \mathrm{e}-04 \backslash \mathrm{Dipol}$ $\mathrm{e}=0 ., 0 ., 0.5579937 \backslash \mathrm{PG}=\mathrm{C} 02 \mathrm{~V} \quad[\mathrm{C} 2$ (C1H1), SGV (C2H4N2) ] \\

\section{Single Point Energy Calculation of H-DHAP ${ }^{+}$in $C_{2 v}$ symmetry}

$1 \backslash 1 \backslash G I N C-N E I L \backslash S P \backslash R B 3 P W 91 \backslash 6-311+G(3 d f, 2 p) \backslash C 3 H 5 N 2(1+) \backslash C H U C K \backslash 31-J u 1-2004 \backslash$ $0 \backslash \backslash \#$ B3PW91/6-311+G (3DF, 2P) GEOM=CHECK GUESS=READ SCF=TIGHT \#P GFINPUT

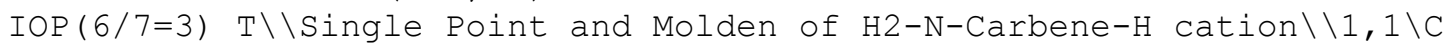
$, 0 ., 0.1 .1401483839 \backslash \mathrm{H}, 0.0 ., 2.2205437336 \backslash \mathrm{N}, 1.0710515936,0 ., 0.347013933$ $2 \backslash \mathrm{N},-1.0710515936,0.0 .3470139332 \backslash \mathrm{C}, 0.6809400271,0 .,-0.9749692056 \backslash \mathrm{C},-0$ $.6809400271,0 \ldots,-0.9749692056 \backslash \mathrm{H}, 1.3907258348,0 .,-1.7880133615 \backslash \mathrm{H},-1.3907$ $258348,0 .,-1.7880133615 \backslash \mathrm{H}, 2.0297961505,0 ., 0.6780140445 \backslash \mathrm{H},-2.0297961505$ , $0 ., 0.6780140445 \backslash \backslash$ Version=x86-Linux-G98RevA.11.1 State=1-A1 \HF=-226.58 $54071 \backslash \mathrm{RMSD}=2.232 e-09 \backslash \mathrm{Dipole}=0 ., 0 ., 0.5416845 \backslash \mathrm{PG}=\mathrm{C} 02 \mathrm{~V} \quad[\mathrm{C} 2(\mathrm{C} 1 \mathrm{H} 1), \mathrm{SGV}(\mathrm{C} 2 \mathrm{H} 4$ N2) $] \backslash \backslash$ Q

\section{Geometry Optimization of $\mathrm{HP}\left(\mathrm{Ph}_{2}\right)_{2}^{+2}$ in $C_{2}$ symmetry}

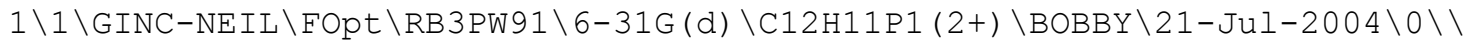
\# B3PW91/6-31G(D) OPT FREQ=NORAMAN POP=(FULL, NBO) TEST \\Geom and Freq of $\mathrm{Ph} 2 \mathrm{P}-\mathrm{H}-2+-\mathrm{C} 2 \backslash \backslash 2,1 \backslash \mathrm{P}, 0 ., 0 ., 0.8942245724 \backslash \mathrm{H}, 0,0 ., 2.293122674 \backslash \mathrm{C}, 1.5740$ $280681,-0.1681432688,0.238164677 \backslash \mathrm{C},-1.5740280681,0.1681432688,0.238164$ $677 \backslash \mathrm{C}, 2.6204187107,-0.2798570515,1.2178933721 \backslash \mathrm{C},-2.6204187107,0.279857$ $0515,1.2178933721 \backslash \mathrm{C}, 1.8783895363,-0.2006132086,-1.1583963267 \backslash \mathrm{C},-1.8783$ $895363,0.2006132086,-1.1583963267 \backslash \mathrm{C}, 3.9244972478,-0.4190475475,0.79393$ $15852 \backslash \mathrm{C},-3.9244972478,0.4190475475,0.7939315852 \backslash \mathrm{C}, 3.1903409986,-0.3405$ $686773,-1.5495547462 \backslash \mathrm{C},-3.1903409986,0.3405686773,-1.5495547462 \backslash \mathrm{C}, 4.21$ $1568358,-0.4495987565,-0.581423179 \backslash \mathrm{C},-4.211568358,0.4495987565,-0.5814$ $23179 \backslash \mathrm{H}, 2.4005416941,-0.2563967171,2.2824116308 \backslash \mathrm{H},-2.4005416941,0.2563$ $967171,2.2824116308 \backslash \mathrm{H}, 1.099926233,-0.1176094483,-1.9077460596 \backslash \mathrm{H},-1.099$ $926233,0.1176094483,-1.9077460596 \backslash \mathrm{H}, 4.7252576677,-0.504573501,1.522182$ $3358 \backslash \mathrm{H},-4.7252576677,0.504573501,1.5221823358 \backslash \mathrm{H}, 3.441718598,-0.3673462$ $548,-2.6055115333 \backslash \mathrm{H},-3.441718598,0.3673462548,-2.6055115333 \backslash \mathrm{H}, 5.242759$ $805,-0.5596294532,-0.9082742978 \backslash \mathrm{H},-5.242759805,0.5596294532,-0.9082742$ 978 \Version=x86-Linux-G98RevA.11. $1 \backslash$ State=1-A \HF=-804.324589 RMSD=8.92 $4 e-09 \backslash \mathrm{RMSF}=6.678 e-06 \backslash \mathrm{Dipole}=0 ., 0 ., 0.4046717 \backslash \mathrm{PG}=\mathrm{C} 02 \quad[\mathrm{C} 2(\mathrm{P} 1 \mathrm{H} 1), \mathrm{X}(\mathrm{C} 12 \mathrm{H} 10)$ ]$\backslash \backslash @$

\section{Single Point Energy Calculation of $\mathrm{HP}\left(\mathrm{Ph}_{2}\right)_{2}{ }^{+2}$ in $C_{2}$ symmetry}

$1 \backslash 1 \backslash G I N C-N E I L \backslash S P \backslash R B 3 P W 91 \backslash 6-311+G(3 d f, 2 p) \backslash C 12 H 11 P 1(2+) \backslash B O B B Y \backslash 22-J u l-200$ $4 \backslash 0 \backslash \backslash \#$ B3PW91/6-311+G(3DF,2P) SCF=TIGHT \#P GFINPUT IOP $(6 / 7=3)$ TEST $\backslash$ Si

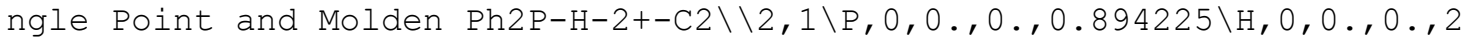
$.293123 \backslash \mathrm{C}, 0,0.000072,1.582983,0.238165 \backslash \mathrm{C}, 0,-0.000072,-1.582983,0.23816$ $5 \backslash \mathrm{C}, 0,0.000055,2.635321,1.217893 \backslash \mathrm{C}, 0,-0.000055,-2.635321,1.217893 \backslash \mathrm{C}, 0$, $0.000043,1.889072,-1.158396 \backslash \mathrm{C}, 0,-0.000043,-1.889072,-1.158396 \backslash \mathrm{C}, 0,0 ., 3$ $.946806,0.793932 \backslash \mathrm{C}, 0,0 .,-3.946806,0.793932 \backslash \mathrm{C}, 0,-0.000087,3.208467,-1.5$ $49555 \backslash \mathrm{C}, 0,0.000087,-3.208467,-1.549555 \backslash \mathrm{C}, 0,-0.000101,4.235498,-0.58142$ 
$3 \backslash \mathrm{C}, 0,0.000101,-4.235498,-0.581423 \backslash \mathrm{H}, 0,0.000073,2.414195,2.282412 \backslash \mathrm{H}, \mathrm{O}$, $-0.000073,-2.414195,2.282412 \backslash \mathrm{H}, 0,0.000161,1.106196,-1.907746 \backslash \mathrm{H}, 0,-0.00$ $0161,-1.106196,-1.907746 \backslash \mathrm{H}, 0,0.000023,4.752121,1.522182 \backslash \mathrm{H}, 0,-0.000023$, $-4.752121,1.522182 \backslash \mathrm{H}, 0,-0.000151,3.461267,-2.605512 \backslash \mathrm{H}, 0,0.000151,-3.46$ $1267,-2.605512 \backslash \mathrm{H}, 0,-0.000178,5.272544,-0.908274 \backslash \mathrm{H}, 0,0.000178,-5.272544$ ,$-0.908274 \backslash \backslash$ Version=x86-Linux-G98RevA.11.1 \State=1-A \HF=-804.4905452\R $\mathrm{MSD}=3.670 \mathrm{e}-09 \backslash \mathrm{Dipole}=0 ., 0 ., 0.3876025 \backslash \mathrm{PG}=\mathrm{C} 02 \quad[\mathrm{C} 2(\mathrm{P} 1 \mathrm{H} 1), \mathrm{X}(\mathrm{C} 12 \mathrm{H} 10)] \backslash \backslash @$

\section{Geometry Optimization of $\mathrm{HP}\left(\mathrm{NMe}_{2}\right)_{2}{ }^{+2}$ in $C_{2}$ symmetry}

$1 \backslash 1 \backslash G I N C-N E I L \backslash F O p t \backslash R B 3 P W 91 \backslash 6-31 G(d) \backslash C 4 H 13 N 2 P 1(2+) \backslash B O B B Y \backslash 21-J u l-2004 \backslash 0 \backslash$ $\backslash \#$ B3PW91/6-31G(D) OPT FREQ=NORAMAN POP=(FULL, NBO) TEST \\Geom and Freq of $(\mathrm{Me} 2 \mathrm{~N}) 2 \mathrm{P}-\mathrm{H}-2+-\mathrm{C} 2 \backslash \backslash 2,1 \backslash \mathrm{P}, 0 ., 0.0 .6638852248 \backslash \mathrm{H}, 0,0 ., 2.0621908469 \backslash \mathrm{N}$, $1.4363337079,-0.1045468738,-0.0113449703 \backslash \mathrm{N},-1.4363337079,0.1045468738$, $-0.0113449703 \backslash \mathrm{C}, 2.5989636065,-0.5333033898,0.8355034004 \backslash \mathrm{C},-2.598963606$ $5,0.5333033898,0.8355034004 \backslash \mathrm{C}, 1.7525448131,0.141354921,-1.4455637283 \backslash \mathrm{C}$ $,-1.7525448131,-0.141354921,-1.4455637283 \backslash \mathrm{H}, 2.3070604934,-0.6966737239$ $, 1.8760162468 \backslash \mathrm{H},-2.3070604934,0.6966737239,1.8760162468 \backslash \mathrm{H}, 0.9481358448$ $, 0.6844248076,-1.9412739884 \backslash \mathrm{H},-0.9481358448,-0.6844248076,-1.941273988$ $4 \backslash \mathrm{H}, 2.9854649077,-1.468265488,0.4189539925 \backslash \mathrm{H},-2.9854649077,1.468265488$ $, 0.4189539925 \backslash \mathrm{H}, 3.3516351986,0.2572853425,0.781374954 \backslash \mathrm{H},-3.3516351986$, $-0.2572853425,0.781374954 \backslash \mathrm{H}, 2.6586251712,0.7540286407,-1.466737043 \backslash \mathrm{H},-$ $2.6586251712,-0.7540286407,-1.466737043 \backslash \mathrm{H}, 1.9443536975,-0.8163041972,-$ $1.9387920116 \backslash \mathrm{H},-1.9443536975,0.8163041972,-1.9387920116 \backslash \backslash$ Version $=x 86-\mathrm{L}$ inux-G98RevA.11.1 \State=1-A \HF=-610.2360584 $\backslash \mathrm{RMSD}=3.200 e-09 \backslash \mathrm{RMSF}=1.803 e$ $-05 \backslash \mathrm{Dipole}=0 ., 0 ., 0.4908849 \backslash \mathrm{PG}=\mathrm{C} 02 \quad[\mathrm{C} 2(\mathrm{P} 1 \mathrm{H} 1), \mathrm{X}(\mathrm{C} 4 \mathrm{H} 12 \mathrm{~N} 2)] \backslash \backslash @$

\section{Single Point Energy Calculation of $\mathrm{HP}\left(\mathrm{NMe}_{2}\right)_{2}{ }^{+2}$ in $C_{2}$ symmetry}

$1 \backslash 1 \backslash G I N C-N E I L \backslash S P \backslash R B 3 P W 91 \backslash 6-311+G(3 d f, 2 p) \backslash C 4 H 13 N 2 P 1(2+) \backslash B O B B Y \backslash 21-J u l-20$ $04 \backslash 0 \backslash \backslash \#$ B3PW91/6-311+G(3DF, 2P) SCF=TIGHT \#P GFINPUT IOP(6/7=3) TEST \\S

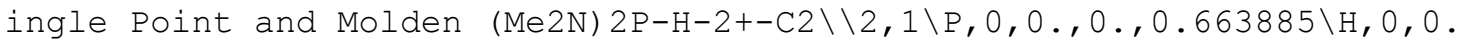
$, 0 ., 2.062191 \backslash \mathrm{N}, 0,0.1 .440134,-0.011345 \backslash \mathrm{N}, 0,0 .,-1.440134,-0.011345 \backslash \mathrm{C}, 0$, $0.343224,2.630821,0.835503 \backslash \mathrm{C}, 0,-0.343224,-2.630821,0.835503 \backslash \mathrm{C}, 0,-0.268$ $208,1.737659,-1.445564 \backslash \mathrm{C}, 0,0.268208,-1.737659,-1.445564 \backslash \mathrm{H}, 0,0.527354,2$ $.351548,1.876016 \backslash \mathrm{H}, 0,-0.527354,-2.351548,1.876016 \backslash \mathrm{H}, 0,-0.751449,0.8959$ $48,-1.941274 \backslash \mathrm{H}, 0,0.751449,-0.895948,-1.941274 \backslash \mathrm{H}, 0,1.247661,3.084177,0$. $418954 \backslash \mathrm{H}, 0,-1.247661,-3.084177,0.418954 \backslash \mathrm{H}, 0,-0.499919,3.324114,0.78137$ $5 \backslash \mathrm{H}, 0,0.499919,-3.324114,0.781375 \backslash \mathrm{H}, 0,-0.945043,2.596871,-1.466737 \backslash \mathrm{H}, 0$ $, 0.945043,-2.596871,-1.466737 \backslash \mathrm{H}, 0,0.672999,1.998483,-1.938792 \backslash \mathrm{H}, 0,-0.6$ $72999,-1.998483,-1.938792 \backslash \backslash$ Version=x86-Linux-G98RevA.11.1 $\backslash$ State $=1-A \backslash H F$ $=-610.3697334 \backslash \mathrm{RMSD}=4.838 \mathrm{e}-09 \backslash \mathrm{Dipole}=0 ., 0 ., 0.4574318 \backslash \mathrm{PG}=\mathrm{C} 02 \quad[\mathrm{C} 2(\mathrm{P} 1 \mathrm{H} 1), \mathrm{X}$ (C4H12N2) ] \\

\section{Geometry Optimization of $\mathrm{Ph}_{2} \mathrm{PH}-\mathrm{PMe}_{3}{ }^{+2}$ in $C_{1}$ symmetry}

$1 \backslash 1 \backslash G I N C-N E I L \backslash F O p t \backslash R B 3 P W 91 \backslash 6-31 G(d) \backslash C 15 H 20 P 2(2+) \backslash B O B B Y \backslash 19-J u 1-2004 \backslash 0 \backslash \backslash$ \# B3PW91/6-31G(D) OPT FREQ=NORAMAN POP=(FULL, NBO) TEST \\Geom and Freq of $\mathrm{Ph} 2 \mathrm{P}-\mathrm{PMe} 3-\mathrm{H}-2+\backslash \backslash 2,1 \backslash \mathrm{P}, 0.0023076206,0.1734753081,-0.6569186681 \backslash \mathrm{C}, 1.4$ $735620822,-0.7766586603,-0.3259438475 \backslash \mathrm{C},-1.5935083592,-0.5364944766,-0$ $.3110636893 \backslash \mathrm{C}, 2.6066075211,-0.5609521479,-1.1360807222 \backslash \mathrm{C},-2.4195387715$ 
$,-0.8363095023,-1.4117819311 \backslash C, 1.4910043845,-1.7304690741,0.7079065877$ $\backslash C,-2.004350212,-0.8348821536,1.0028218413 \backslash C, 3.757940192,-1.3011882238$ $,-0.8955858929 \backslash \mathrm{C},-3.6522271421,-1.4411001196,-1.1867785551 \backslash \mathrm{C}, 2.6537226$ $24,-2.4599828881,0.9304572621 \backslash C,-3.2402231671,-1.4370744066,1.20475302$ $43 \backslash \mathrm{C}, 3.7821234762,-2.2431184807,0.1364472984 \backslash \mathrm{C},-4.0603588194,-1.739680$ $5341,0.113606155 \backslash \mathrm{H}, 2.5857699487,0.1447744822,-1.9634500678 \backslash \mathrm{H},-2.108322$ $6494,-0.6165842296,-2.4298097354 \backslash \mathrm{H}, 0.6069454668,-1.9310913936,1.305685$ $58 \backslash \mathrm{H},-1.3715408254,-0.6177435502,1.8594020146 \backslash \mathrm{H}, 4.6309650057,-1.156941$ $1649,-1.5248475428 \backslash \mathrm{H},-4.291859806,-1.6812401049,-2.0306296042 \backslash \mathrm{H}, 2.6747$ $008994,-3.2094207263,1.7157489859 \backslash \mathrm{H},-3.5645314632,-1.6755744088,2.2131$ $58961 \backslash \mathrm{H}, 4.6830085614,-2.8238261923,0.31230925 \backslash \mathrm{H},-5.0235387587,-2.21306$ $83854,0.2804211573 \backslash \mathrm{P}, 0.1642015611,2.2142424429,0.2938206039 \backslash \mathrm{C}, 0.380491$ $9861,2.0216331068,2.0823681042 \backslash C, 1.614332892,3.0361017758,-0.418571421$ $1 \backslash \mathrm{C},-1.351068695,3.134247757,-0.0794690244 \backslash \mathrm{H}, 1.2391124839,1.3794512054$ $, 2.3020769005 \backslash \mathrm{H}, 0.5669478501,3.0135845801,2.5117459171 \backslash \mathrm{H},-0.5229808148$ $, 1.6112391488,2.5419047874 \backslash \mathrm{H}, 1.4890811582,3.1860818874,-1.4955252705 \backslash \mathrm{H}$ $, 1.7231550756,4.0180176307,0.0582019097 \backslash \mathrm{H}, 2.5194390249,2.4515368138,-0$ $.2269972515 \backslash \mathrm{H},-1.4610317829,3.2792502614,-1.1587973966 \backslash \mathrm{H},-2.2261690258$ $, 2.605374244,0.310224019 \backslash \mathrm{H},-1.287483888,4.1183685458,0.4008320973 \backslash \mathrm{H}, 0$. $0096358617,0.5376132592,-2.0136948846 \backslash \backslash$ Version=x86-Linux-G98RevA.11.1\} $\mathrm{HF}=-1265.4712374 \backslash \mathrm{RMSD}=7.750 \mathrm{e}-09 \backslash \mathrm{RMSF}=2.811 \mathrm{e}-06 \backslash \mathrm{Dipole}=0.1379067,2.3637$ $546,0.2008499 \backslash \mathrm{PG}=\mathrm{C} 01 \quad[\mathrm{X}(\mathrm{C} 15 \mathrm{H} 20 \mathrm{P} 2)] \backslash \backslash @$

\section{Single Point Energy Calculation of $\mathrm{Ph}_{2} \mathrm{PH}_{-} \mathrm{PMe}_{3}{ }^{+2}$ in $C_{1}$ symmetry}

$1 \backslash 1 \backslash G I N C-N E I L \backslash S P \backslash R B 3 P W 91 \backslash 6-311+G(3 d f, 2 p) \backslash C 15 H 20 P 2(2+) \backslash B O B B Y \backslash 24-J u 1-200$ $4 \backslash 0 \backslash \backslash \#$ B3PW91/6-311+G(3DF, 2P) SCF=TIGHT \#P GFINPUT IOP(6/7=3) TEST \\Si ngle Point and Molden Ph2P-PMe3-H-2+\\2,1\P,0,0.006166,0.144679,-0.663 $831 \backslash \mathrm{C}, 0,1.488425,-0.766888,-0.278027 \backslash \mathrm{C}, 0,-1.581956,-0.574516,-0.301995$ $\backslash C, 0,2.626014,-0.56894,-1.086327 \backslash C, 0,-2.392354,-0.934984,-1.396122 \backslash C, 0$ $, 1.510201,-1.674317,0.796684 \backslash C, 0,-2.001192,-0.821744,1.019838 \backslash C, 0,3.78$ $6091,-1.279806,-0.803051 \backslash \mathrm{C}, 0,-3.61782,-1.548678,-1.156266 \backslash \mathrm{C}, 0,2.681675$ $,-2.375076,1.06167 \backslash \mathrm{C}, 0,-3.229653,-1.433899,1.236502 \backslash \mathrm{C}, 0,3.814443,-2.17$ $5341,0.269387 \backslash \mathrm{C}, 0,-4.034208,-1.796645,0.152113 \backslash \mathrm{H}, 0,2.60259,0.099622,-1$ $.94394 \backslash \mathrm{H}, 0,-2.074458,-0.754998,-2.419872 \backslash \mathrm{H}, 0,0.623431,-1.862545,1.3944$ $79 \backslash \mathrm{H}, 0,-1.380299,-0.557545,1.871904 \backslash \mathrm{H}, 0,4.663003,-1.149439,-1.429932 \backslash \mathrm{H}$ $, 0,-4.245309,-1.835406,-1.994673 \backslash \mathrm{H}, 0,2.706326,-3.089135,1.879161 \backslash \mathrm{H}, 0,-$ $3.560256,-1.633265,2.25134 \backslash \mathrm{H}, 0,4.722315,-2.733603,0.478801 \backslash \mathrm{H}, 0,-4.9916$ $43,-2.277381,0.330579 \backslash \mathrm{P}, 0,0.127403,2.227255,0.19816 \backslash \mathrm{C}, 0,0.328873,2.116$ $242,1.995343 \backslash \mathrm{C}, 0,1.571788,3.039947,-0.536088 \backslash \mathrm{C}, 0,-1.397984,3.106189,-0$ $.229005 \backslash \mathrm{H}, 0,1.194993,1.49782,2.25086 \backslash \mathrm{H}, 0,0.495873,3.12878,2.382611 \backslash \mathrm{H}, 0$ $,-0.572729,1.712134,2.464033 \backslash \mathrm{H}, 0,1.454939,3.140837,-1.619683 \backslash \mathrm{H}, 0,1.660$ $851,4.043308,-0.10175 \backslash \mathrm{H}, 0,2.483783,2.478584,-0.310769 \backslash \mathrm{H}, 0,-1.499447,3$. $202243,-1.314614 \backslash \mathrm{H}, 0,-2.268714,2.581129,0.175357 \backslash \mathrm{H}, 0,-1.354219,4.11119$ $5,0.208343 \backslash \mathrm{H}, 0,0.021375,0.449377,-2.035115 \backslash \backslash$ Version=x86-Linux-G98RevA. $11.1 \backslash \mathrm{HF}=-1265.7159309 \backslash \mathrm{RMSD}=3.895 \mathrm{e}-09 \backslash \mathrm{Dipole}=0.1117055,2.3347586,0.0882$ $133 \backslash \mathrm{PG}=\mathrm{CO} 1 \quad[\mathrm{X}(\mathrm{C} 15 \mathrm{H} 20 \mathrm{P} 2)] \backslash \backslash \mathrm{Q}$

\section{Geometry Optimization of $\left(\mathrm{Me}_{2} \mathrm{~N}\right)_{2} \mathrm{PH}-\mathrm{PMe}_{3}{ }^{+2}$ in $C_{1}$ symmetry}

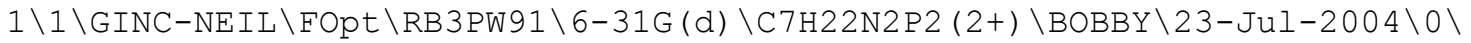
$\backslash \#$ B3PW91/6-31G(D) OPT FREQ=NORAMAN POP=(FULL, NBO) TEST \\Geom and Freq

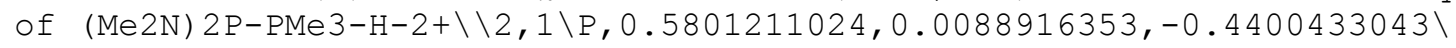


$\mathrm{P},-1.6488586677,-0.0592841662,-0.0890211649 \backslash \mathrm{N}, 1.064315824,1.5316262287$ $,-0.0676234714 \backslash \mathrm{N}, 1.2748445549,-1.3788883889,0.0801636486 \backslash \mathrm{C}, 2.230681560$ $5,2.0894170778,-0.7985554469 \backslash \mathrm{C}, 2.0257332411,-2.2787801306,-0.826357187$ $2 \backslash \mathrm{C}, 0.8319239822,2.1618676625,1.2449906947 \backslash \mathrm{C}, 1.4747280954,-1.647221651$ $8,1.5170267598 \backslash \mathrm{H}, 2.3002753592,1.6646442909,-1.8034058547 \backslash \mathrm{H}, 1.818138216$ $4,-2.046725253,-1.873469446 \backslash \mathrm{H}, 0.6749094482,3.2330117161,1.0906846178 \backslash \mathrm{H}$ $, 1.1131207303,-2.651673927,1.7563041881 \backslash \mathrm{H}, 2.0757567458,3.1673787286,-0$ $.8955794743 \backslash \mathrm{H}, 3.100138167,-2.1806476299,-0.6430756676 \backslash \mathrm{H}, 3.1633395755,1$ $.903283952,-0.2573273676 \backslash \mathrm{H}, 1.7167214894,-3.3095295927,-0.6326927948 \backslash \mathrm{H}$, $-0.0567191193,1.7507852089,1.7317587307 \backslash \mathrm{H}, 2.5394943256,-1.5855056319,1$ $.7636680455 \backslash \mathrm{H}, 1.6909952908,2.0225178346,1.9095431496 \backslash \mathrm{H}, 0.9386647739,-0$ $.9205954081,2.1345181668 \backslash \mathrm{C},-2.0971533239,-0.54005802,1.6019884168 \backslash \mathrm{C},-2$ $.2175216441,-1.3443503949,-1.2363900257 \backslash \mathrm{C},-2.3746592054,1.5408101728,-$ $0.5299583336 \backslash \mathrm{H},-2.0245362266,-1.0588959972,-2.2752762354 \backslash \mathrm{H},-2.10302658$ $24,1.8188366045,-1.5530664008 \backslash \mathrm{H},-3.1916865759,-0.613253309,1.637746686$ $\backslash \mathrm{H},-1.7356769916,-2.3023421729,-1.018204814 \backslash \mathrm{H},-3.3005783809,-1.4615103$ $837,-1.1066448501 \backslash \mathrm{H},-3.4665583873,1.4495157242,-0.4701019175 \backslash \mathrm{H},-2.0481$ $819758,2.3247201173,0.1585683064 \backslash \mathrm{H},-1.6796800999,-1.5199273866,1.85085$ $41912 \backslash \mathrm{H},-1.7792267226,0.2013888065,2.3398520764 \backslash \mathrm{H}, 0.6688615313,-0.0888$ $614999,-1.8329368069 \backslash \backslash V e r s i o n=x 86-L i n u x-G 98 R e v A .11 .1 \backslash H F=-1071.385046 \backslash R$ $\mathrm{MSD}=5.609 e-09 \backslash \mathrm{RMSF}=6.058 e-06 \backslash \mathrm{Dipole}=-0.9416425,-0.0953142,-0.113665 \backslash \mathrm{PG}$ $=\mathrm{C} 01 \quad[\mathrm{X}(\mathrm{C} 7 \mathrm{H} 22 \mathrm{~N} 2 \mathrm{P} 2)] \backslash \backslash \mathrm{C}$

\section{Single Point Energy Calculation of $\left(\mathrm{Me}_{2} \mathrm{~N}\right)_{2} \mathrm{PH}-\mathrm{PMe}_{3}{ }^{+2}$ in $C_{1}$ symmetry}

$1 \backslash 1 \backslash G I N C-N E I L \backslash S P \backslash R B 3 P W 91 \backslash 6-311+G(3 d f, 2 p) \backslash C 7 H 22 N 2 P 2(2+) \backslash B O B B Y \backslash 25-J u l-20$ $04 \backslash 0 \backslash \backslash \#$ B3PW91/6-311+G(3DF, 2P) SCF=TIGHT \#P GFINPUT IOP (6/7=3) TEST \\S ingle Point and Molden of (Me2N)2P-PMe3-H-2+\\2,1\P,0,0.577621,0.00432 $6,-0.443388 \backslash \mathrm{P}, 0,-1.648722,-0.075772,-0.078424 \backslash \mathrm{N}, 0,1.050193,1.539097,-0$ $.107211 \backslash \mathrm{N}, 0,1.287542,-1.365491,0.10324 \backslash \mathrm{C}, 0,2.207539,2.090941,-0.856758$ $\backslash \mathrm{C}, 0,2.041516,-2.278449,-0.787518 \backslash \mathrm{C}, 0,0.819303,2.195988,1.192539 \backslash \mathrm{C}, 0,1$ $.497614,-1.600376,1.5445 \backslash \mathrm{H}, 0,2.275472,1.644795,-1.852418 \backslash \mathrm{H}, 0,1.826172$, $-2.071326,-1.838288 \backslash \mathrm{H}, 0,0.65188,3.262051,1.015644 \backslash \mathrm{H}, 0,1.146308,-2.6024$ $72,1.807798 \backslash \mathrm{H}, 0,2.042455,3.165099,-0.976547 \backslash \mathrm{H}, 0,3.115979,-2.166834,-0$. $61248 \backslash \mathrm{H}, 0,3.144748,1.924994,-0.316829 \backslash \mathrm{H}, 0,1.74279,-3.307374,-0.569541 \backslash$ $\mathrm{H}, 0,-0.062972,1.78789,1.693203 \backslash \mathrm{H}, 0,2.563109,-1.523858,1.783735 \backslash \mathrm{H}, 0,1.6$ $83181,2.078875,1.855149 \backslash \mathrm{H}, 0,0.958434,-0.865099,2.148898 \backslash \mathrm{C}, 0,-2.083512$, $-0.523164,1.625225 \backslash \mathrm{C}, 0,-2.212089,-1.390734,-1.194093 \backslash \mathrm{C}, 0,-2.391186,1.5$ $0777,-0.550288 \backslash \mathrm{H}, 0,-2.027307,-1.126512,-2.240064 \backslash \mathrm{H}, 0,-2.127609,1.76560$ $7,-1.580764 \backslash \mathrm{H}, 0,-3.177137,-0.605208,1.668737 \backslash \mathrm{H}, 0,-1.720519,-2.339407,-$ $0.957646 \backslash \mathrm{H}, 0,-3.293335,-1.514562,-1.055719 \backslash \mathrm{H}, 0,-3.481884,1.408186,-0.4$ $82303 \backslash \mathrm{H}, 0,-2.067999,2.309487,0.119018 \backslash \mathrm{H}, 0,-1.655948,-1.493601,1.893188$ $\backslash \mathrm{H}, 0,-1.768228,0.237111,2.344837 \backslash \mathrm{H}, 0,0.659669,-0.123259,-1.834277 \backslash \backslash \mathrm{Ver}$

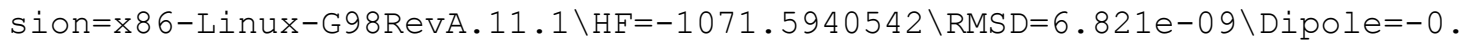
$9499491,-0.0942711,-0.1082388 \backslash \mathrm{PG}=\mathrm{C} 01 \quad[\mathrm{X}(\mathrm{C} 7 \mathrm{H} 22 \mathrm{~N} 2 \mathrm{P} 2)] \backslash \backslash \mathrm{C}$

\section{Geometry Optimization of $\mathrm{Ph}_{2} \mathrm{PH}-\mathrm{DHAP}{ }^{+2}$ in $C_{l}$ symmetry}

$1 \backslash 1 \backslash G I N C-N E I L \backslash F O p t \backslash R B 3 P W 91 \backslash 6-31 G(d) \backslash C 17 H 17 N 2 P 1(2+) \backslash B O B B Y \backslash 19-J u l-2004 \backslash 0$ $\backslash \backslash \#$ B3PW91/6-31G(D) OPT FREQ=NORAMAN POP=(FULL, NBO) TEST \\Geom and Fre $\mathrm{q}$ of $\mathrm{Ph} 2 \mathrm{P}-\mathrm{DHAP}-\mathrm{H}-2+\backslash \backslash 2,1 \backslash \mathrm{P}, 0.4302270836,0.0394807067,-0.7838500045 \backslash \mathrm{C}, 1$ $.1841282727,1.5203082338,-0.1834858553 \backslash \mathrm{C}, 1.1284178134,-1.5189309103,-0$ $.3254988812 \backslash \mathrm{C}, 1.1233429013,2.6804909435,-0.9847906924 \backslash \mathrm{C}, 1.9311724074,-$ $2.1760004366,-1.2778785159 \backslash \mathrm{C}, 1.8813023314,1.5197567289,1.0406900926 \backslash \mathrm{C}$, 
$0.9647449793,-2.0419109098,0.9723410635 \backslash C, 1.7467833611,3.8397525166,-0$ $.5412055046 \backslash \mathrm{C}, 2.5719783363,-3.3576108091,-0.9200901204 \backslash \mathrm{C}, 2.4959023565$, $2.6909018939,1.465824062 \backslash \mathrm{C}, 1.611468275,-3.2248673759,1.3068866301 \backslash \mathrm{C}, 2$. $4273847648,3.8442979049,0.6797834979 \backslash C, 2.4137936698,-3.8772985069,0.36$ $53604171 \backslash \mathrm{H}, 0.6125170686,2.6776937418,-1.9452484705 \backslash \mathrm{H}, 2.0589824443,-1.7$ $781187766,-2.2817989243 \backslash \mathrm{H}, 1.963968798,0.6164340161,1.6382905118 \backslash \mathrm{H}, 0.33$ $61543056,-1.5442671564,1.7068568775 \backslash \mathrm{H}, 1.7154910971,4.7358508632,-1.153$ $3933541 \backslash \mathrm{H}, 3.1907336193,-3.8739701722,-1.6474669937 \backslash \mathrm{H}, 3.0429007731,2.70$ $14573804,2.403520027 \backslash \mathrm{H}, 1.4904468057,-3.6418907115,2.3020204296 \backslash \mathrm{H}, 2.921$ $6051716,4.7518882088,1.0144449569 \backslash \mathrm{H}, 2.9156295439,-4.8015401547,0.63682$ $42578 \backslash \mathrm{N},-1.2842749173,0.0190596218,-0.3262923278 \backslash \mathrm{C},-2.0479781793,-1.08$ $37470903,-0.6390746714 \backslash \mathrm{C},-1.8908116011,1.1075075623,0.2546040354 \backslash \mathrm{C},-3$. $3843740021,-1.1237525329,-0.3892230229 \backslash \mathrm{C},-3.2230767554,1.1199854447,0$. $5333962343 \backslash \mathrm{C},-4.0381333752,-0.006398436,0.21366385 \backslash \mathrm{H},-1.5283898249,-1$. $9239065696,-1.087907989 \backslash \mathrm{H},-1.2500525075,1.9516876116,0.4827341989 \backslash \mathrm{H},-3$ $.9404983784,-2.0172718254,-0.6547173233 \backslash \mathrm{H},-3.6515257505,2.00147707,0.9$ $996229217 \backslash \mathrm{N},-5.3392405044,-0.0178407057,0.4652883908 \backslash \mathrm{H},-5.9215258723,-$ $0.8142354836,0.2358238662 \backslash \mathrm{H},-5.8083823135,0.7724982543,0.8910068758 \backslash \mathrm{H}$, $0.3868733844,0.1105653672,-2.1836499558 \backslash \backslash$ Version=x86-Linux-G98RevA.11. $1 \backslash \mathrm{HF}=-1108.0118935 \backslash \mathrm{RMSD}=4.101 \mathrm{e}-09 \backslash \mathrm{RMSF}=9.895 e-07 \backslash \mathrm{Dipole}=-2.9658024,0.0$ $545264,-0.044173 \backslash \mathrm{PG}=\mathrm{C} 01 \quad[\mathrm{X}(\mathrm{C} 17 \mathrm{H} 17 \mathrm{~N} 2 \mathrm{P} 1)] \backslash \backslash @$

\section{Single Point Energy Calculation of $\mathrm{Ph}_{2} \mathrm{PH}-\mathrm{DHAP}{ }^{+2}$ in $C_{1}$ symmetry}

$1 \backslash 1 \backslash G I N C-N E I L \backslash S P \backslash R B 3 P W 91 \backslash 6-311+G(3 d f, 2 p) \backslash C 17 H 17 N 2 P 1(2+) \backslash B O B B Y \backslash 22-J u l-2$ $004 \backslash 0 \backslash \backslash \#$ B3PW91/6-311+G(3DF, 2P) SCF=TIGHT \#P GFINPUT IOP $(6 / 7=3) \quad \mathrm{TEST} \backslash \backslash$ Single Point and Molden Ph2P-DHAP-H-2+\\2,1\P,0,0.430987,0.012114,-0.7 $84333 \backslash \mathrm{C}, 0,1.159365,1.530089,-0.248727 \backslash \mathrm{C}, 0,1.153796,-1.51296,-0.25717 \backslash \mathrm{C}$ $, 0,1.081051,2.652817,-1.100288 \backslash \mathrm{C}, 0,1.969004,-2.197891,-1.178846 \backslash \mathrm{C}, 0,1$. $854162,1.594764,0.975092 \backslash \mathrm{C}, 0,0.996298,-1.981086,1.0622 \backslash \mathrm{C}, 0,1.684555,3$. $840548,-0.707334 \backslash \mathrm{C}, 0,2.628441,-3.351938,-0.768766 \backslash \mathrm{C}, 0,2.448667,2.79340$ $9,1.34908 \backslash \mathrm{C}, 0,1.661717,-3.137401,1.449126 \backslash \mathrm{C}, 0,2.362705,3.909901,0.5130$ $58 \backslash \mathrm{C}, 0,2.476399,-3.817229,0.538088 \backslash \mathrm{H}, 0,0.572138,2.599444,-2.060282 \backslash \mathrm{H}, 0$ , 2.092147,-1.842412,-2.199127\H,0,1.950521,0.720031, $1.611888 \backslash \mathrm{H}, 0,0.358$ $253,-1.462101,1.773425 \backslash \mathrm{H}, 0,1.63971,4.70827,-1.358327 \backslash \mathrm{H}, 0,3.256945,-3.8$ $89467,-1.472049 \backslash \mathrm{H}, 0,2.993663,2.854129,2.286035 \backslash \mathrm{H}, 0,1.545692,-3.512274$, $2.461477 \backslash \mathrm{H}, 0,2.84134,4.839327,0.808098 \backslash \mathrm{H}, 0,2.992824,-4.72027,0.850463 \backslash$ $\mathrm{N}, 0,-1.283802,-0.016443,-0.32829 \backslash \mathrm{C}, 0,-2.028721,-1.144349,-0.593207 \backslash \mathrm{C}, 0$ $,-1.909202,1.086317,0.203539 \backslash \mathrm{C}, 0,-3.364746,-1.195358,-0.343375 \backslash \mathrm{C}, 0,-3$. $242012,1.089071,0.479984 \backslash \mathrm{C}, 0,-4.037878,-0.063537,0.209099 \backslash \mathrm{H}, 0,-1.49457$ $8,-1.994728,-1.004107 \backslash \mathrm{H}, 0,-1.282809,1.950139,0.395105 \backslash \mathrm{H}, 0,-3.905637,-2$ $.108712,-0.570005 \backslash \mathrm{H}, 0,-3.685738,1.983002,0.906549 \backslash \mathrm{N}, 0,-5.33909,-0.0853$ $55,0.459489 \backslash \mathrm{H}, 0,-5.9078,-0.90054,0.264558 \backslash \mathrm{H}, 0,-5.821933,0.715074,0.849$ $542 \backslash \mathrm{H}, 0,0.389093,0.020956,-2.185953 \backslash \backslash$ Version=x86-Linux-G98RevA.11.1\HF $=-1108.2806879 \backslash \mathrm{RMSD}=3.725 e-09 \backslash \mathrm{Dipole}=-2.8835589,0.0027999,-0.0534403 \backslash \mathrm{P}$ $\mathrm{G}=\mathrm{C} 01 \quad[\mathrm{X}(\mathrm{C} 17 \mathrm{H} 17 \mathrm{~N} 2 \mathrm{P} 1)] \backslash \backslash \mathrm{Q}$

\section{Geometry Optimization of $\left(\mathrm{Me}_{2} \mathrm{~N}\right)_{2} \mathrm{PH}-\mathrm{DHAP}^{+2}$ in $C_{l}$ symmetry}

$1 \backslash 1 \backslash G I N C-N E I L \backslash F O p t \backslash R B 3 P W 91 \backslash 6-31 G(d) \backslash C 9 H 19 N 4 P 1(2+) \backslash B O B B Y \backslash 24-J u 1-2004 \backslash 0 \backslash$ \\# B3PW91/6-31G(D) OPT FREQ=NORAMAN POP=(FULL, NBO) TEST \Geom and Freq of $(\mathrm{Me} 2 \mathrm{~N}) 2 \mathrm{P}-\mathrm{DHAP}-\mathrm{H}-2+\backslash \backslash 2,1 \backslash \mathrm{P},-1.1488231375,-0.0906140462,-0.554346186$

$7 \backslash \mathrm{N},-1.9468246278,-1.2779328808,0.1964260732 \backslash \mathrm{N},-1.6930782301,1.3787839$ 
$541,-0.1259884134 \backslash \mathrm{C},-2.2430102571,-2.5611293969,-0.4793410351 \backslash \mathrm{C},-0.960$ $8163665,2.6347997347,-0.4039276334 \backslash C,-2.2337773355,-1.2655809278,1.644$ $3628601 \backslash \mathrm{C},-3.1425519265,1.601894542,0.1056358401 \backslash \mathrm{H},-2.067086087,-2.493$ $0995908,-1.555404209 \backslash \mathrm{H}, 0.0954078789,2.4528072601,-0.6023995223 \backslash \mathrm{H},-2.03$ $29478145,-0.2828363318,2.0755501227 \backslash \mathrm{H},-3.6723678061,0.6590638719,0.239$ $246863 \backslash \mathrm{H},-1.6237794989,-3.3598398251,-0.0611398286 \backslash \mathrm{H},-1.4023034309,3.1$ $35447234,-1.2712991037 \backslash \mathrm{H},-3.2987153075,-2.7972198402,-0.3208224055 \backslash \mathrm{H},-$ $1.0497685468,3.287577398,0.4687891759 \backslash \mathrm{H},-3.2908875529,-1.5013644668,1$. $7968684898 \backslash \mathrm{H},-3.2575198365,2.2073060257,1.0086911789 \backslash \mathrm{H},-1.6231405293,-$ $2.0195024838,2.1508979178 \backslash \mathrm{H},-3.5733959712,2.1360460579,-0.746601232 \backslash \mathrm{N}$, $0.5976656876,0.0077847295,-0.2973144236 \backslash \mathrm{C}, 3.3651682395,-0.0853102514,0$ $.1879983217 \backslash \mathrm{C}, 1.4671551483,-0.4394656112,-1.2682247553 \backslash \mathrm{C}, 1.1107501207$, $0.3995978683,0.9208808541 \backslash \mathrm{C}, 2.8109959011,-0.4883404519,-1.0651520356 \backslash \mathrm{C}$ ,2.444152644,0.3670761101,1.1836312759\N,4.6680247733,-0.1270611178,0. $416758405 \backslash \mathrm{H}, 1.0341631399,-0.742804512,-2.2161545394 \backslash \mathrm{H}, 0.3947357599,0.7$ $49374437,1.6565192024 \backslash \mathrm{H}, 3.45041819,-0.8371824137,-1.8699440151 \backslash \mathrm{H}, 2.795$ $3360152,0.6921722729,2.1579787143 \backslash \mathrm{H}, 5.3251424466,-0.4449147166,-0.2863$ $849519 \backslash \mathrm{H}, 5.0661726702,0.157769953,1.3041083233 \backslash \mathrm{H},-1.1260268852,-0.4918$ $621258,-1.8976610218 \backslash \backslash$ Version=x86-Linux-G98RevA.11.1 \HF=-913.9355353\R $\mathrm{MSD}=6.892 \mathrm{e}-09 \backslash \mathrm{RMSF}=3.823 e-06 \backslash \mathrm{Dipole}=1.5678949,-0.189056,-0.0163411 \backslash \mathrm{PG}=$ $\mathrm{C} 01[\mathrm{X}(\mathrm{C} 9 \mathrm{H} 19 \mathrm{~N} 4 \mathrm{P} 1)] \backslash \backslash \mathrm{Q}$

\section{Single Point Energy Calculation of $\left(\mathrm{Me}_{2} \mathrm{~N}\right)_{2} \mathrm{PH}-\mathrm{DHAP}^{+2}$ in $C_{1}$ symmetry}

$1 \backslash 1 \backslash G I N C-N E I L \backslash S P \backslash R B 3 P W 91 \backslash 6-311+G(3 d f, 2 p) \backslash C 9 H 19 N 4 P 1(2+) \backslash B O B B Y \backslash 26-J u l-20$ $04 \backslash 0 \backslash \backslash \#$ B3PW91/6-311+G(3DF, 2P) SCF=TIGHT \#P GFINPUT IOP (6/7=3) TEST \\S ingle Point and Molden of (Me2N)2P-DHAP-H-2+\\2,1\P,0,-1.148167,-0.087 $949,-0.556132 \backslash \mathrm{N}, 0,-1.947347,-1.278329,0.188514 \backslash \mathrm{N}, 0,-1.692617,1.379702$, $-0.122073 \backslash \mathrm{C}, 0,-2.242993,-2.558517,-0.493169 \backslash \mathrm{C}, 0,-0.959738,2.636744,-0$. $393673 \backslash \mathrm{C}, 0,-2.236061,-1.272188,1.63614 \backslash \mathrm{C}, 0,-3.142322,1.602136,0.108751$ $\backslash \mathrm{H}, 0,-2.065743,-2.485864,-1.568712 \backslash \mathrm{H}, 0,0.096687,2.455373,-0.591646 \backslash \mathrm{H}, 0$ $,-2.035539,-0.291367,2.071827 \backslash \mathrm{H}, 0,-3.67251,0.658856,0.237629 \backslash \mathrm{H}, 0,-1.62$ $445,-3.359174,-0.07768 \backslash \mathrm{H}, 0,-1.400056,3.141247,-1.259403 \backslash \mathrm{H}, 0,-3.298943$, $-2.795053,-0.336961 \backslash \mathrm{H}, 0,-1.049609,3.285753,0.481756 \backslash \mathrm{H}, 0,-3.293409,-1.5$ $0839,1.786335 \backslash \mathrm{H}, 0,-3.258256,2.203654,1.014281 \backslash \mathrm{H}, 0,-1.62621,-2.028437,2$ $.140146 \backslash \mathrm{H}, 0,-3.572009,2.140075,-0.741687 \backslash \mathrm{N}, 0,0.598029,0.008937,-0.2965$ $5 \backslash \mathrm{C}, 0,3.364918,-0.08689,0.191725 \backslash \mathrm{C}, 0,1.468602,-0.434298,-1.26833 \backslash \mathrm{C}, 0,1$ $.109716,0.39535,0.923956 \backslash \mathrm{C}, 0,2.812184,-0.484359,-1.063834 \backslash \mathrm{C}, 0,2.44279$, $0.361387,1.188187 \backslash \mathrm{N}, 0,4.667485,-0.129928,0.421889 \backslash \mathrm{H}, 0,1.036699,-0.7334$ $27,-2.218092 \backslash \mathrm{H}, 0,0.392883,0.742098,1.660231 \backslash \mathrm{H}, 0,3.452509,-0.829855,-1$. $869352 \backslash \mathrm{H}, 0,2.792857,0.682177,2.164362 \backslash \mathrm{H}, 0,5.325389,-0.444881,-0.281825$ $\backslash \mathrm{H}, 0,5.064614,0.150964,1.310949 \backslash \mathrm{H}, 0,-1.123822,-0.483376,-1.901145 \backslash \backslash V e r$

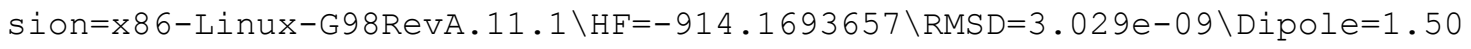
$36106,-0.1671477,-0.0179199 \backslash \mathrm{PG}=\mathrm{CO} 1 \quad[\mathrm{X}(\mathrm{C} 9 \mathrm{H} 19 \mathrm{~N} 4 \mathrm{P} 1)] \backslash \backslash \mathrm{Q}$

\section{Geometry Optimization of $\mathrm{Ph}_{2} \mathrm{PH}-$ Carbene ${ }^{+2}$ in $C_{1}$ symmetry}

$1 \backslash 1 \backslash$ GINC-NEIL \FOpt \RB3PW91 \6-31G(d) \C15H15N2P1(2+) \BOBBY $17-J u 1-2004 \backslash 0$ $\backslash \backslash \#$ B3PW91/6-31G(D) OPT FREQ=NORAMAN POP=(FULL, NBO) TEST \\Geom and Fre $\mathrm{q}$ of $\mathrm{Ph} 2 \mathrm{P}-\mathrm{CarbH}-\mathrm{H}-2+\backslash \backslash 2,1 \backslash \mathrm{P}, 0.0117003215,0.0747817835,-0.7728792183 \backslash \mathrm{C}$, $1.6438553657,-0.4312169628,-0.2962662943 \backslash \mathrm{C},-1.4025548624,-0.8836778997$ $,-0.2757604587 \backslash \mathrm{C}, 2.1031255196,-0.2601942175,1.0252202591 \backslash \mathrm{C},-2.55151390$ $29,-0.8626416554,-1.0933116562 \backslash C, 2.4339547813,-1.0847010328,-1.2618584$ $437 \backslash \mathrm{C},-1.3555692163,-1.6620560406,0.8949757075 \backslash \mathrm{C}, 3.3590179431,-0.74552$ $47015,1.3688091756 \backslash \mathrm{C},-3.6593087322,-1.6134976268,-0.7155507248 \backslash \mathrm{C}, 3.687$ 
$8939717,-1.5619021117,-0.895254222 \backslash \mathrm{C},-2.4741187292,-2.4075087171,1.251$ $1781695 \backslash \mathrm{C}, 4.1463474145,-1.3945289036,0.4125628673 \backslash \mathrm{C},-3.6196728363,-2.3$ $806576722,0.4520165322 \backslash \mathrm{H}, 1.4962706371,0.2397812979,1.7770466028 \backslash \mathrm{H},-2.5$ $767004067,-0.2981351977,-2.0235338122 \backslash \mathrm{H}, 2.0830396194,-1.2224424362,-2$. $2816903752 \backslash \mathrm{H},-0.4566613592,-1.7083032068,1.5035696729 \backslash \mathrm{H}, 3.7261280296,-$ $0.6194513756,2.3828728306 \backslash \mathrm{H},-4.5456382775,-1.6169713285,-1.3428301433 \backslash$ $\mathrm{H}, 4.3069853698,-2.0636535084,-1.632603435 \backslash \mathrm{H},-2.4473079479,-3.021734816$ $8,2.1460489787 \backslash \mathrm{H}, 5.1266309963,-1.7706411841,0.6907211066 \backslash \mathrm{H},-4.48435656$ $59,-2.9752365859,0.7325302453 \backslash \mathrm{C},-0.2479623203,1.7904608207,-0.17797776$ $81 \backslash \mathrm{N}, 0.6382275586,2.8005791013,-0.1481634228 \backslash \mathrm{N},-1.4050625612,2.3009757$ $973,0.2744038322 \backslash \mathrm{C}, 0.0465257633,3.9447249811,0.3187318398 \backslash \mathrm{C},-1.2575536$ $387,3.6256185404,0.588615643 \backslash \mathrm{H}, 1.6142827282,2.7209649044,-0.4211940596$ $\backslash \mathrm{H},-2.263633751,1.7636048096,0.3714594641 \backslash \mathrm{H}, 0.5846177754,4.8761686077$, $0.4259030689 \backslash \mathrm{H},-2.0682461023,4.2269191153,0.9753195372 \backslash \mathrm{H},-0.0178696754$ $, 0.2003390594,-2.1708980316 \backslash \backslash$ Version=x86-Linux-G98RevA.11.1 \HF=-1030.5 $935088 \backslash \mathrm{RMSD}=2.838 e-09 \backslash \mathrm{RMSF}=3.257 e-06 \backslash \mathrm{Dipole}=-0.3463285,2.727555,-0.046$ $923 \backslash \mathrm{PG}=\mathrm{C} 01 \quad[\mathrm{X}(\mathrm{C} 15 \mathrm{H} 15 \mathrm{~N} 2 \mathrm{P} 1)] \backslash \backslash \mathrm{C}$

\section{Single Point Energy Calculation of $\mathrm{Ph}_{2} \mathrm{PH}-$ Carbene $^{+2}$ in $C_{1}$ symmetry}

$1 \backslash 1 \backslash G I N C-N E I L \backslash S P \backslash R B 3 P W 91 \backslash 6-311+G(3 d f, 2 p) \backslash C 15 H 15 N 2 P 1(2+) \backslash B O B B Y \backslash 21-J u l-2$ $004 \backslash 0 \backslash \backslash \#$ B3PW91/6-311+G(3DF, 2P) SCF=TIGHT \#P GFINPUT IOP $(6 / 7=3)$ TEST \ Single Point and Molden Ph2P-CarbH-H-2+\\2,1\P,0,0.011217,0.061943,-0. $774021 \backslash \mathrm{C}, 0,1.617278,-0.521535,-0.297316 \backslash \mathrm{C}, 0,-1.448499,-0.81302,-0.2548$ $31 \backslash \mathrm{C}, 0,2.092378,-0.354176,1.019033 \backslash \mathrm{C}, 0,-2.599398,-0.744493,-1.067035 \backslash \mathrm{C}$ $, 0,2.366534,-1.23066,-1.256182 \backslash \mathrm{C}, 0,-1.435742,-1.574348,0.927929 \backslash \mathrm{C}, 0,3$. $323028,-0.899343,1.364227 \backslash \mathrm{C}, 0,-3.742874,-1.430128,-0.671982 \backslash \mathrm{C}, 0,3.5957$ $9,-1.767246,-0.888096 \backslash \mathrm{C}, 0,-2.589796,-2.254361,1.301392 \backslash \mathrm{C}, 0,4.069806,-1$ $.603703,0.414647 \backslash \mathrm{C}, 0,-3.736886,-2.179918,0.507466 \backslash \mathrm{H}, 0,1.516849,0.18869$ $8,1.765723 \backslash \mathrm{H}, 0,-2.600234,-0.194091,-2.006008 \backslash \mathrm{H}, 0,2.003095,-1.365749,-2$ $.271975 \backslash \mathrm{H}, 0,-0.537055,-1.658192,1.532819 \backslash \mathrm{H}, 0,3.701994,-0.776845,2.3743$ $6 \backslash \mathrm{H}, 0,-4.631713,-1.396883,-1.294822 \backslash \mathrm{H}, 0,4.183528,-2.312309,-1.620342 \backslash \mathrm{H}$ $, 0,-2.590146,-2.855047,2.205804 \backslash \mathrm{H}, 0,5.030586,-2.026368,0.694014 \backslash \mathrm{H}, 0,-4$ $.629947,-2.723801,0.801615 \backslash \mathrm{C}, 0,-0.154755,1.798002,-0.205306 \backslash \mathrm{N}, 0,0.7833$ $3,2.760534,-0.1959 \backslash \mathrm{N}, 0,-1.280911,2.375602,0.24448 \backslash \mathrm{C}, 0,0.255089,3.94134$ $9,0.255551 \backslash \mathrm{C}, 0,-1.062374,3.695432,0.536808 \backslash \mathrm{H}, 0,1.752303,2.625505,-0.47$ $2355 \backslash \mathrm{H}, 0,-2.165911,1.885645,0.354254 \backslash \mathrm{H}, 0,0.841876,4.844816,0.345244 \backslash \mathrm{H}$, $0,-1.838217,4.344461,0.917798 \backslash \mathrm{H}, 0,-0.019666,0.166954,-2.173705 \backslash \backslash$ Versio $\mathrm{n}=\mathrm{x} 86-\mathrm{Linux}-\mathrm{G} 98 \mathrm{RevA} .11 .1 \backslash \mathrm{HF}=-1030.837197 \backslash \mathrm{RMSD}=4.156 \mathrm{e}-09 \backslash \mathrm{Dipole}=-0.2004$ $664,2.6312088,-0.1030596 \backslash \mathrm{PG}=\mathrm{C} 01 \quad[\mathrm{X}(\mathrm{C} 15 \mathrm{H} 15 \mathrm{~N} 2 \mathrm{P} 1)] \backslash \backslash \mathrm{C}$

\section{Geometry Optimization of $\left(\mathrm{Me}_{2} \mathrm{~N}\right)_{2} \mathrm{PH}-$ Carbene $^{+2}$ in $C_{S}$ symmetry}

$1 \backslash 1 \backslash G I N C-N E I L \backslash F O p t \backslash R B 3 P W 91 \backslash 6-31 G(d) \backslash C 7 H 17 N 4 P 1(2+) \backslash B O B B Y \backslash 16-J u 1-2004 \backslash 0 \backslash$ \\# B3PW91/6-31G(D) OPT FREQ=NORAMAN POP=(FULL, NBO) TEST \Geom and Freq of $(\mathrm{Me} 2 \mathrm{~N}) 2 \mathrm{P}-\mathrm{CarbH}-\mathrm{H}-2+-\mathrm{Cs} \backslash \backslash 2,1 \backslash \mathrm{P}, 0.5069359106,0 .,-0.6222075722 \backslash \mathrm{C}, 0.16$ $32049883,0.1 .1624598899 \backslash \mathrm{H}, 1.8893493246,0 .,-0.8164781493 \backslash \mathrm{N}, 0.024043331$ $8,-1.4875220051,-1.1217210651 \backslash \mathrm{N}, 0.0240433318,1.4875220051,-1.121721065$ $1 \backslash \mathrm{C}, 0.79948279,-2.1918547194,-2.1743807285 \backslash \mathrm{C}, 0.79948279,2.1918547194,-$ $2.1743807285 \backslash \mathrm{C},-1.3955749718,-1.9007295831,-1.0524596805 \backslash \mathrm{C},-1.39557497$ $18,1.9007295831,-1.0524596805 \backslash \mathrm{H}, 1.8529656023,-1.9028985484,-2.14264331$ $16 \backslash \mathrm{H}, 1.8529656023,1.9028985484,-2.1426433116 \backslash \mathrm{H},-1.4350837491,-2.974434$ $4002,-0.8484178954 \backslash \mathrm{H},-1.4350837491,2.9744344002,-0.8484178954 \backslash \mathrm{H}, 0.7358$ 
$363019,-3.264595983,-1.974620726 \backslash \mathrm{H}, 0.3918071613,1.9865561106,-3.168810$ $8523 \backslash \mathrm{H}, 0.3918071613,-1.9865561106,-3.1688108523 \backslash \mathrm{H}, 0.7358363019,3.26459$ $5983,-1.974620726 \backslash \mathrm{H},-1.924596925,-1.3736703143,-0.2530047856 \backslash \mathrm{H},-1.9055$ $422044,1.7065200183,-2.0014950608 \backslash \mathrm{H},-1.9055422044,-1.7065200183,-2.001$ $4950608 \backslash \mathrm{H},-1.924596925,1.3736703143,-0.2530047856 \backslash \mathrm{N}, 0.0917541097,1.073$ $1310174,1.9712054707 \backslash \mathrm{N}, 0.0917541097,-1.0731310174,1.9712054707 \backslash \mathrm{C},-0.03$ $56359732,0.6862316752,3.2755603367 \backslash \mathrm{C},-0.0356359732,-0.6862316752,3.275$ $5603367 \backslash \mathrm{H}, 0.1421518405,2.0357998603,1.6478103626 \backslash \mathrm{H}, 0.1421518405,-2.035$ $7998603,1.6478103626 \backslash \mathrm{H},-0.114060144,1.3934050661,4.0898880605 \backslash \mathrm{H},-0.114$ $060144,-1.3934050661,4.0898880605 \backslash \backslash$ Version=x86-Linux-G98RevA.11.1\Stat $e=1-A^{\prime} \backslash \mathrm{HF}=-836.5144836 \backslash \mathrm{RMSD}=4.543 e-09 \backslash \mathrm{RMSF}=8.820 \mathrm{e}-06 \backslash \mathrm{Dipole}=0.1286597$, $0 ., 1.288443 \backslash \mathrm{PG}=\mathrm{CS} \quad[\mathrm{SG}(\mathrm{C} 1 \mathrm{H} 1 \mathrm{P} 1), \mathrm{X}(\mathrm{C} 6 \mathrm{H} 16 \mathrm{~N} 4)] \backslash \backslash \mathrm{Q}$

\section{Single Point Energy Calculation of $\left(\mathrm{Me}_{2} \mathrm{~N}\right)_{2} \mathrm{PH}-\mathrm{Carbene}{ }^{+2}$ in $C_{s}$ symmetry}

$1 \backslash 1 \backslash G I N C-N E I L \backslash S P \backslash R B 3 P W 91 \backslash 6-311+G(3 d f, 2 p) \backslash C 7 H 17 N 4 P 1(2+) \backslash B O B B Y \backslash 22-J u 1-20$ $04 \backslash 0 \backslash \backslash \#$ B3PW91/6-311+G(3DF, 2P) SCF=TIGHT \#P GFINPUT IOP(6/7=3) TEST \\S ingle Point and Molden (Me2N) 2P-CarbH-H-2+\\2,1 $1 \mathrm{P}, 0,0 ., 0.802575,0 . \backslash \mathrm{C}, 0$ $,-0.86078,-0.798127,0 . \backslash \mathrm{H}, 0,-0.949027,1.826369,0 . \backslash \mathrm{N}, 0,0.689881,0.884817$ $, 1.487522 \backslash \mathrm{N}, 0,0.689881,0.884817,-1.487522 \backslash \mathrm{C}, 0,0.753609,2.190703,2.1918$ $55 \backslash \mathrm{C}, 0,0.753609,2.190703,-2.191855 \backslash \mathrm{C}, 0,1.746712,-0.065562,1.90073 \backslash \mathrm{C}, 0$, $1.746712,-0.065562,-1.90073 \backslash \mathrm{H}, 0,-0.083165,2.831516,1.902899 \backslash \mathrm{H}, 0,-0.083$ $165,2.831516,-1.902899 \backslash \mathrm{H}, 0,1.648461,-0.248704,2.974434 \backslash \mathrm{H}, 0,1.648461,-0$ $.248704,-2.974434 \backslash \mathrm{H}, 0,0.676776,1.995634,3.264596 \backslash \mathrm{H}, 0,1.697784,2.704146$ $,-1.986556 \backslash \mathrm{H}, 0,1.697784,2.704146,1.986556 \backslash \mathrm{H}, 0,0.676776,1.995634,-3.264$ $596 \backslash \mathrm{H}, 0,1.651878,-1.019501,1.37367 \backslash \mathrm{H}, 0,2.741517,0.348077,-1.70652 \backslash \mathrm{H}, 0$, $2.741517,0.348077,1.70652 \backslash \mathrm{H}, 0,1.651878,-1.019501,-1.37367 \backslash \mathrm{N}, 0,-1.31622$ $,-1.47025,-1.073131 \backslash \mathrm{N}, 0,-1.31622,-1.47025,1.073131 \backslash \mathrm{C}, 0,-2.041338,-2.56$ $1934,-0.686232 \backslash \mathrm{C}, 0,-2.041338,-2.561934,0.686232 \backslash \mathrm{H}, 0,-1.151023,-1.1877$, $-2.0358 \backslash \mathrm{H}, 0,-1.151023,-1.1877,2.0358 \backslash \mathrm{H}, 0,-2.494898,-3.242789,-1.393405$ $\backslash \mathrm{H}, 0,-2.494898,-3.242789,1.393405 \backslash \backslash$ Version=x86-Linux-G98RevA.11.1 \Stat $e=1-A^{\prime} \backslash H F=-836.7215851 \backslash R M S D=3.395 e-09 \backslash D i p o l e=-0.8845539,-0.9008014,0 . \backslash$ $\mathrm{PG}=\mathrm{CS} \quad[\mathrm{SG}(\mathrm{C} 1 \mathrm{H} 1 \mathrm{P} 1), \mathrm{X}(\mathrm{C} 6 \mathrm{H} 16 \mathrm{~N} 4)] \backslash \backslash \mathrm{C}$

Plots of the Laplacian of the electron density for each donor- $\mathrm{PPh}_{2}{ }^{+}$complex.
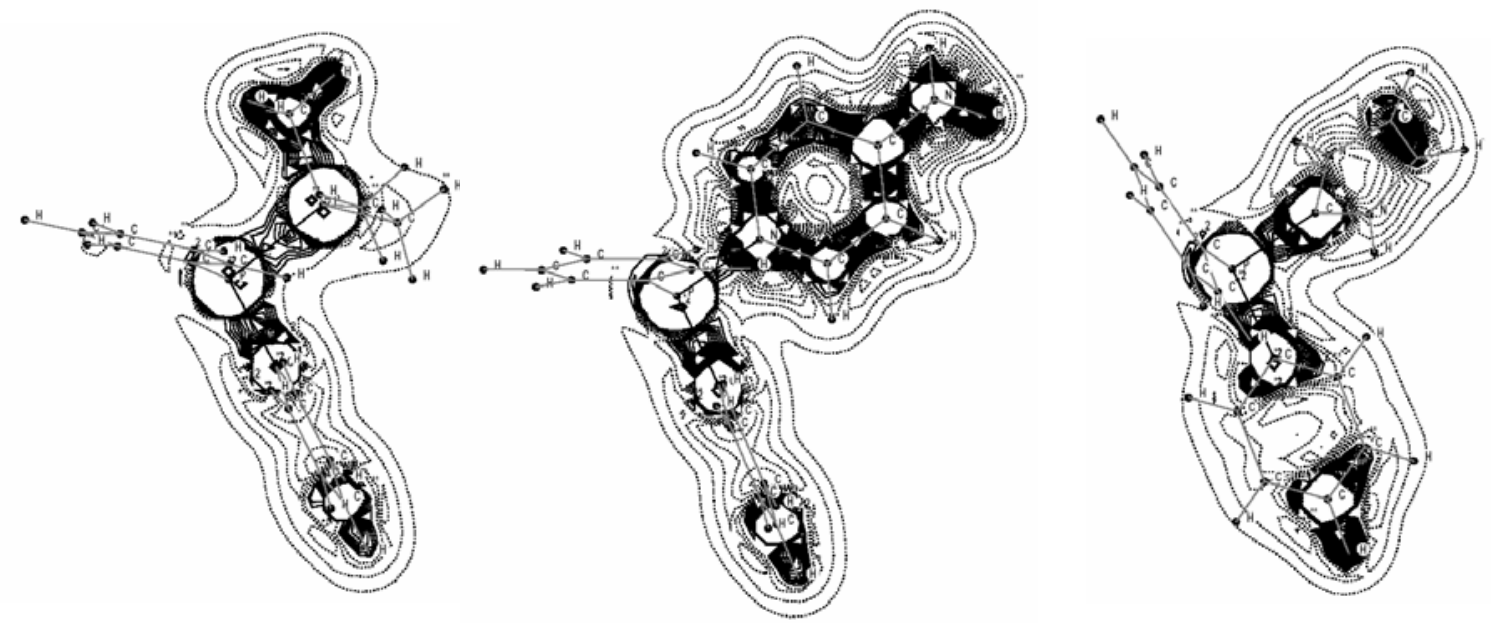
Plots of the Laplacian of the electron density for each donor-P(NMe $)_{2}{ }^{+}$complex.
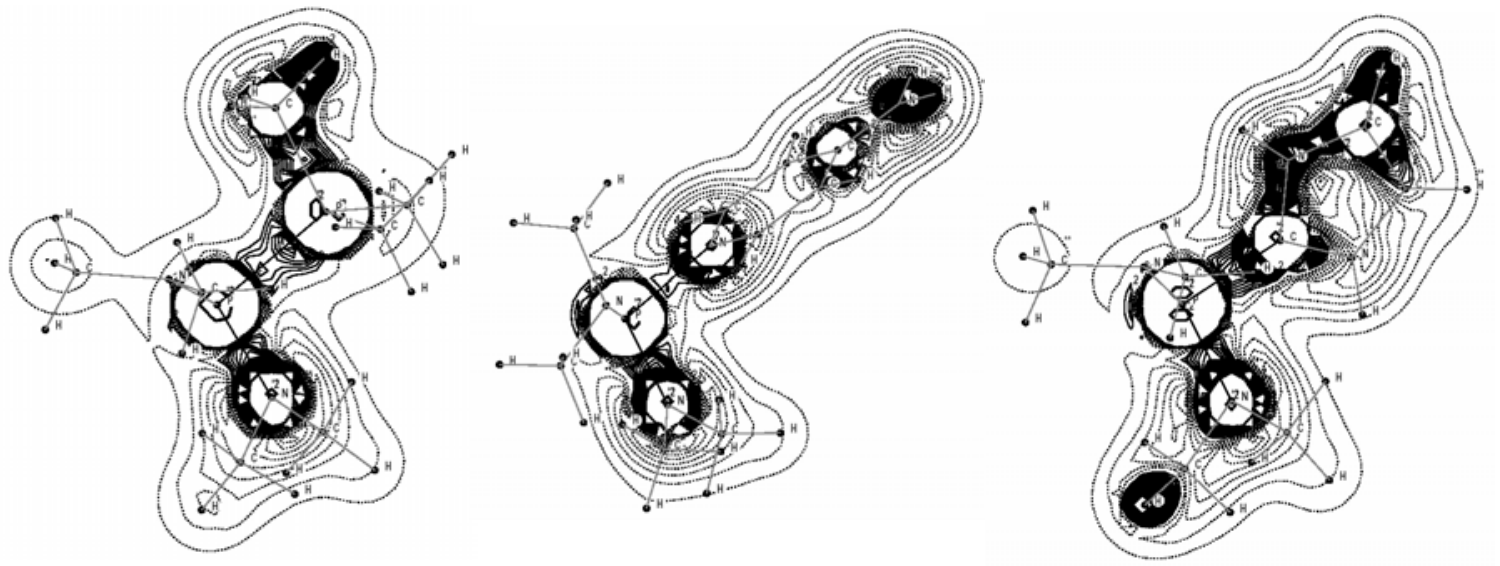